\title{
The Heliospheric Imagers Onboard the STEREO Mission
}

\author{
C.J. Eyles • R.A. Harrison • C.J. Davis • N.R. Waltham • B.M. Shaughnessy • \\ H.C.A. Mapson-Menard • D. Bewsher · S.R. Crothers · J.A. Davies • G.M. Simnett • \\ R.A. Howard · J.D. Moses · J.S. Newmark · D.G. Socker · J.-P. Halain · J.-M. Defise • \\ E. Mazy $\cdot$ P. Rochus
}

Received: 22 September 2008 / Accepted: 21 November 2008 / Published online: 23 December 2008

(C) Springer Science+Business Media B.V. 2008

\begin{abstract}
Mounted on the sides of two widely separated spacecraft, the two Heliospheric Imager (HI) instruments onboard NASA's STEREO mission view, for the first time, the space between the Sun and Earth. These instruments are wide-angle visible-light imagers that incorporate sufficient baffling to eliminate scattered light to the extent that the passage of solar coronal mass ejections (CMEs) through the heliosphere can be detected. Each HI instrument comprises two cameras, HI- 1 and HI-2, which have $20^{\circ}$ and $70^{\circ}$ fields of view and are off-pointed from the Sun direction by $14.0^{\circ}$ and $53.7^{\circ}$, respectively, with their optical axes aligned in the ecliptic plane. This arrangement provides coverage over solar elongation angles from $4.0^{\circ}$ to $88.7^{\circ}$ at the viewpoints of the two spacecraft, thereby allowing the observation of Earth-directed CMEs along the Sun-Earth line to the vicinity of the Earth and beyond. Given the two separated platforms, this also presents the first opportunity to view the structure and evolution of CMEs in three dimensions. The STEREO spacecraft were launched from Cape Canaveral Air Force Base in late October 2006, and the HI instruments have been performing scientific observations since early 2007. The design, development, manufacture, and calibration of these unique instruments are reviewed in this paper. Mission operations, including the initial commissioning phase and the science operations phase,
\end{abstract}

C.J. Eyles $(\varangle) \cdot$ R.A. Harrison · C.J. Davis · N.R. Waltham · B.M. Shaughnessy ·

H.C.A. Mapson-Menard · D. Bewsher · S.R. Crothers · J.A. Davies

Space Science and Technology Department, STFC Rutherford Appleton Laboratory, Chilton, Didcot,

Oxfordshire OX11 0QX, UK

e-mail: cje@star.sr.bham.ac.uk

C.J. Eyles · G.M. Simnett

School of Physics and Astronomy, University of Birmingham, Birmingham B15 2TT, UK

R.A. Howard · J.D. Moses · J.S. Newmark · D.G. Socker

Space Science Division, Naval Research Laboratory, Washington DC, USA

J.-P. Halain · J.-M. Defise · E. Mazy $\cdot$ P. Rochus

Centre Spatial de Liège, Universitè de Liège, ave Prè Aily, 4031 Angleur, Belgium

C.J. Eyles

Laboratorio de Procesado de Imagenes, Universidad de Valencia, 46071 Valencia, Spain 
are described. Data processing and analysis procedures are briefly discussed, and groundtest results and in-orbit observations are used to demonstrate that the performance of the instruments meets the original scientific requirements.

\section{Introduction}

Coronal Mass Ejections (CMEs) are among the most energetic and dramatic transient events in the solar system, releasing some $10^{12}-10^{13} \mathrm{~kg}$ of matter from the Sun at speeds of typically $300-400 \mathrm{~km} \mathrm{~s}^{-1}$, but occasionally up to over $2500 \mathrm{~km} \mathrm{~s}^{-1}$, in discrete eruptions. Such eruptions, which are a major component of coronal evolution, clearly cause severe disturbances in the heliosphere and can impact near-Earth space sufficiently to affect a wide range of human activities. Understanding the CME phenomenon, from launch to arrival at Earth, is clearly a major goal in solar and heliospheric physics. There are currently a number of operational spacecraft making solar and heliospheric observations, including the ESA/NASA Solar and Heliospheric Observatory (SOHO), the Coriolis spacecraft with the U.S. Air Force Solar Mass Ejection Imager (SMEI) onboard, and the NASA Transition Region and Coronal Explorer (TRACE), together with the more recently launched NASA Solar Terrestrial Relations Observatory (STEREO) mission and the Japanese Hinode satellite. Given this fleet of spacecraft, there is the opportunity for a major multi-instrument attack on many aspects of CME physics and this field is developing rapidly (see recent reviews in, e.g., Gopalswamy, Mewaldt, and Torsti, 2006).

CMEs have conventionally been detected by using coronagraphs; these are instruments that occult the Sun's disc to detect the faint outer corona and its eruptions. An example of a traditional coronagraph observation of a CME, using the LASCO instrument on the SOHO spacecraft, is shown in Figure 1. This technique introduces two basic observational challenges. Firstly, the instruments that detect the CMEs do not observe the solar disc, which means that CME onset studies invariably involve back-projection of data to relate CMEs to surface structures and events identified using other instrumentation. This observational disconnection leads to major difficulties in investigating the CME onset process (see Harrison, 2006, and references therein). Secondly, any Earth-directed CMEs are not well viewed by near-Earth spacecraft. The nature of the Thomson scattering process that illuminates CME material means that they are poorly viewed out of the plane of the sky, and any Earth-directed CMEs will be seen as "halo" events coming from behind the occulting disc of the coronagraph. Such events were first identified by Howard et al. (1982) and have been identified routinely in recent years (e.g., Kim et al., 2005; Lara et al., 2006). Determining the speed of Earth-directed events is clearly very difficult and the observational restrictions would suggest that we do not actually detect all Earth-directed CMEs. Indeed, we may only detect a small fraction in this way.

Thus, one "holy grail" for solar physics has been to observe CMEs in interplanetary space, along the Sun - Earth line, and that is the underlying goal of the Heliospheric Imager instruments (Socker et al., 2000; Defise et al., 2003; Harrison, Davis, and Eyles, 2005; Eyles et al., 2007) aboard the NASA STEREO mission.

Occupying solar orbits at approximately $1 \mathrm{AU}$, with one spacecraft leading and the other lagging the Earth, and with each spacecraft separating from the Earth by $22.5^{\circ}$ per year, the two STEREO spacecraft provide vantage points from which one can view the Sun - Earth line. The spacecraft are designated STEREO-A for the leading, or "ahead", spacecraft and STEREO-B for the trailing, or "behind", spacecraft.

The STEREO multi-instrument remote sensing package, known as SECCHI (Sun-Earth Connection Coronal and Heliospheric Investigation; see Howard, Moses, and Socker, 2000; 
Figure 1 An image taken on 4 January 2002 by the LASCO C3 coronagraph aboard SOHO showing a CME off the solar northeast limb. The image also shows stars, Mercury, and streamers. The image of Mercury is saturated, causing blooming along the columns of the CCD detector.

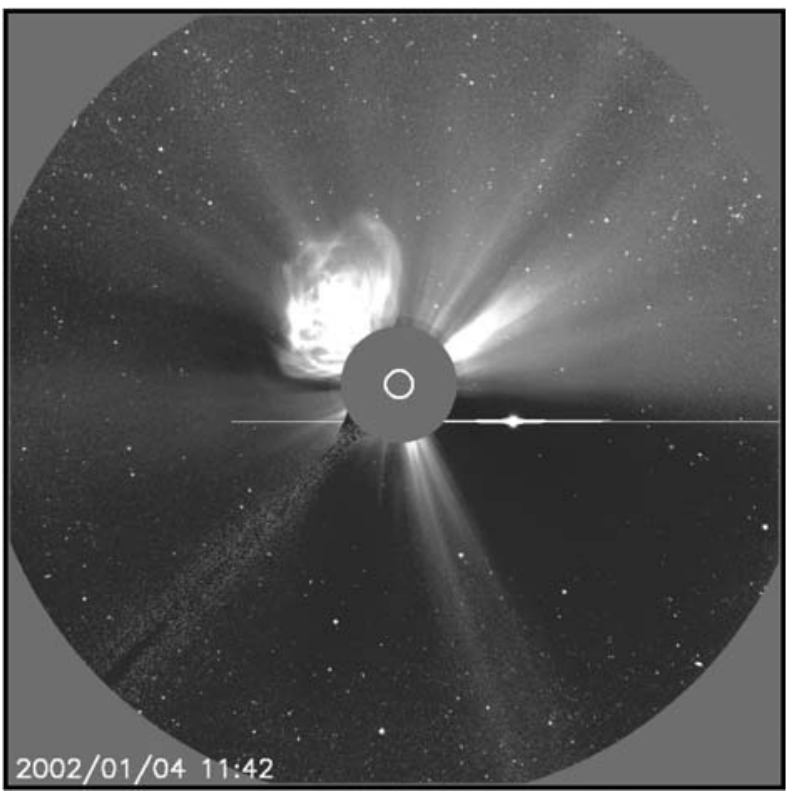

Howard et al., 2008) includes a Heliospheric Imager (HI) on each spacecraft. The same designation is used for the HI instruments on the two spacecraft (i.e., HI-A and HI-B). The other major component of SECCHI is the Sun Centred Instrument Package (SCIP), comprising an Extreme Ultra-Violet Imager (EUVI), which images the chromosphere and lower corona out to $1.7 R_{\odot}$ (solar radii) in four emission lines between 17.1 and $30.4 \mathrm{~nm}$, and a pair of traditional white-light Lyot coronagraphs (COR-1 and COR-2), which cover the coronal regions $1.4 R_{\odot}-4 R_{\odot}$ and $2.5 R_{\odot}-15 R_{\odot}$ in the plane of the sky, respectively, with bandpass from 650 to $750 \mathrm{~nm}$. SCIP also includes a Guide Telescope (GT) whose output error signals are fed into the control loop of the spacecraft attitude control system to maintain the SCIP instruments accurately pointing at Sun centre.

The HI consists of two visible-light cameras (HI-1 and HI-2) mounted on the side of each STEREO spacecraft (see Figure 2), which view space, sheltered from the glare of the Sun by a system of baffles. This system provides us with several important new opportunities for CME research including, for the first time, the ability to pursue the following STEREO primary science objectives:

- to observe geoeffective CMEs along the Sun - Earth line in interplanetary space,

- to detect CMEs in a field of view that includes the Earth, and

- to obtain stereographic views of CMEs in interplanetary space and hence to investigate their structure, evolution, and propagation in the heliosphere.

In addition to the primary objectives summarised here, the instrumentation provides unique opportunities to pursue a number of additional scientific goals, including:

- direct imaging from two different locations of corotating interaction regions (CIRs), regions of plasma compression formed where fast solar wind streams catch up with slow solar wind,

- stellar variability studies, including the search for exoplanets; the HI cameras are able to continuously monitor the light-curves of stars down to 12 th magnitude from a very stable environment, for periods up to 20 and 70 days for HI-1 and HI-2, respectively, 


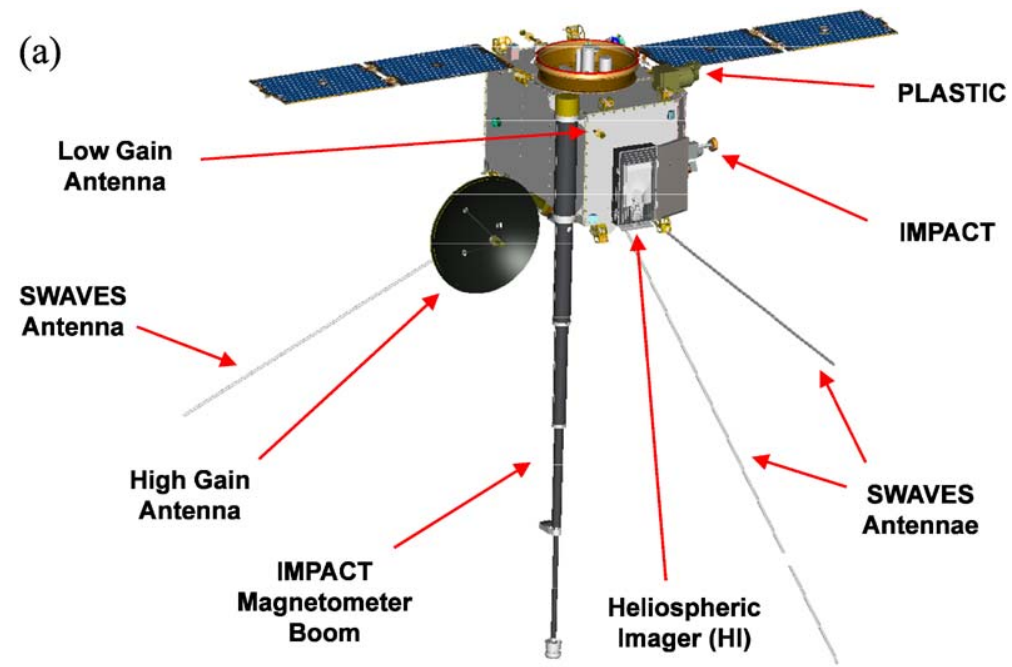

(b)

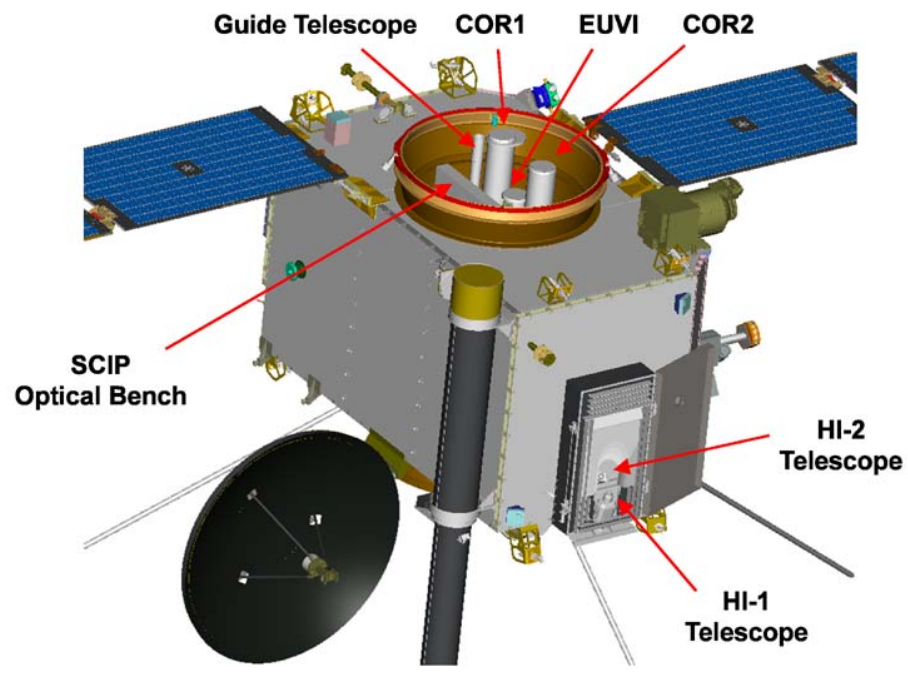

Figure 2 (a) The Heliospheric Imager instrument mounted on the side of one of the STEREO spacecraft. The diagram also shows a number of other instruments and spacecraft subsystems. (b) A close-up of the spacecraft body showing the HI telescopes, together with the SECCHI Sun Centred Instrument Package. (Adapted from diagrams by the Johns Hopkins University Applied Physics Laboratory.)

- observations of comets, including the potential for three-dimensional imaging of cometary tails, and

- observations of asteroids.

The basic instrumental approach is through occultation and a baffle system, with wideangle views of the heliosphere, achieving light rejection levels sufficient to view diffuse density enhancements revealed by Thomson-scattered photospheric light from free electrons in the solar wind plasma. 
The heritage for the HI instrument comes from the SMEI instrument (Eyles et al., 2003; Jackson et al., 2004), operating aboard the Coriolis spacecraft, launched in 2003. The SMEI instrument is also a wide-angle heliospheric imaging system, with three $60^{\circ} \times 3^{\circ}$ field-ofview baffled camera systems. The spacecraft is in a Sun-synchronous polar orbit, with its attitude stabilised with respect to the local vertical. The cameras sweep around the sky as the spacecraft orbits the Earth and consequently almost the entire sky is mapped each orbit.

The basic concept of the SMEI instrument is similar to HI in that a baffle technique is used to enable imaging of faint, diffuse structures in the heliosphere. However, there are significant differences. There are two HI instruments and each is mounted aboard a three-axis stabilised spacecraft, with a fixed field of view looking towards the Sun-Earth line. Thus, the HI instruments allow a continuous view of the heliosphere between the Sun and Earth, from two increasingly separated locations (see Figure 4). This allows a unique capability for the study of Earth-directed CMEs and their three-dimensional structure. The SMEI instrument operates from Earth orbit and consequently does not view the Sun -Earth line.

\section{Scientific Requirements and the Instrument Concept}

The basic design concept for the HI can be seen in Figure 3. The instrument is essentially a box shape, of major dimension about $800 \mathrm{~mm}$. A door was utilised to protect the optical and baffle systems during ground operations, launch, and the initial cruise phase activities. The door is a one-shot system - it is opened once during instrument commissioning and remains open thereafter. The two telescope/camera systems, HI-1 and HI-2, are housed deep within a baffle system as shown in Figure 3. The direction to the Sun is indicated - the Sun remains below the vanes of the forward baffle system. The detectors are charge-coupled detectors (CCDs), which are passively cooled by radiators facing space in the antisunward direction.

The performance specifications for HI are listed in Table 1. The HI-1 and HI-2 telescope boresights are directed at angles of $13.65^{\circ}$ and $53.35^{\circ}$ from the principal axis of the instrument, which in turn is tilted upwards by approximately 20 arc-min to ensure that the Sun is sufficiently below the baffle horizon. Thus, the two optical axes are nominally set to $14.0^{\circ}$ and $53.7^{\circ}$ from the Sun, in the ecliptic plane, with fields of view of $20^{\circ}$ and $70^{\circ}$, respectively. This provides an overlap in solar elongation angle of about $5^{\circ}$ between the two fields of view, with complete coverage along the Sun - Earth line from $4.0^{\circ}$ to $88.7^{\circ}$ elongation.

Both telescopes are designed to image visible light. In the case of HI-2, the camera is designed to have as wide a spectral response as possible to maximise the weak coronal signal at large solar elongations. The bandpass of HI-1 is chosen to approximately match that of COR-2, the outermost of the SCIP Sun-pointing coronagraphs in the SECCHI instrument suite.

The HI detectors are CCDs with $2048 \times 2048$ pixels, where each pixel has a size of $13.5 \times 13.5 \mu \mathrm{m}$. These are usually binned onboard to $1024 \times 1024$ bins, resulting in image bin angular sizes of 70 arc-sec and 4 arc-min, for HI-1 and HI-2, respectively. As discussed later, to obtain sufficient statistical accuracy, long-duration exposures are required. However, the rate of cosmic-ray hits would compromise such images if they were taken as single exposures. Thus, short exposures are taken and cleaned of cosmic rays onboard, and a number of such exposures are then summed to produce an image to be down-linked (see Section 5.3).

For each telescope, Table 1 lists the nominal exposure time and number of exposures per summed image sequence. With a CCD line transfer rate of rather more than $2 \mathrm{~ms}$ per line and an image clear sequence of $124 \mu$ s per line, the readout time of each exposure is 


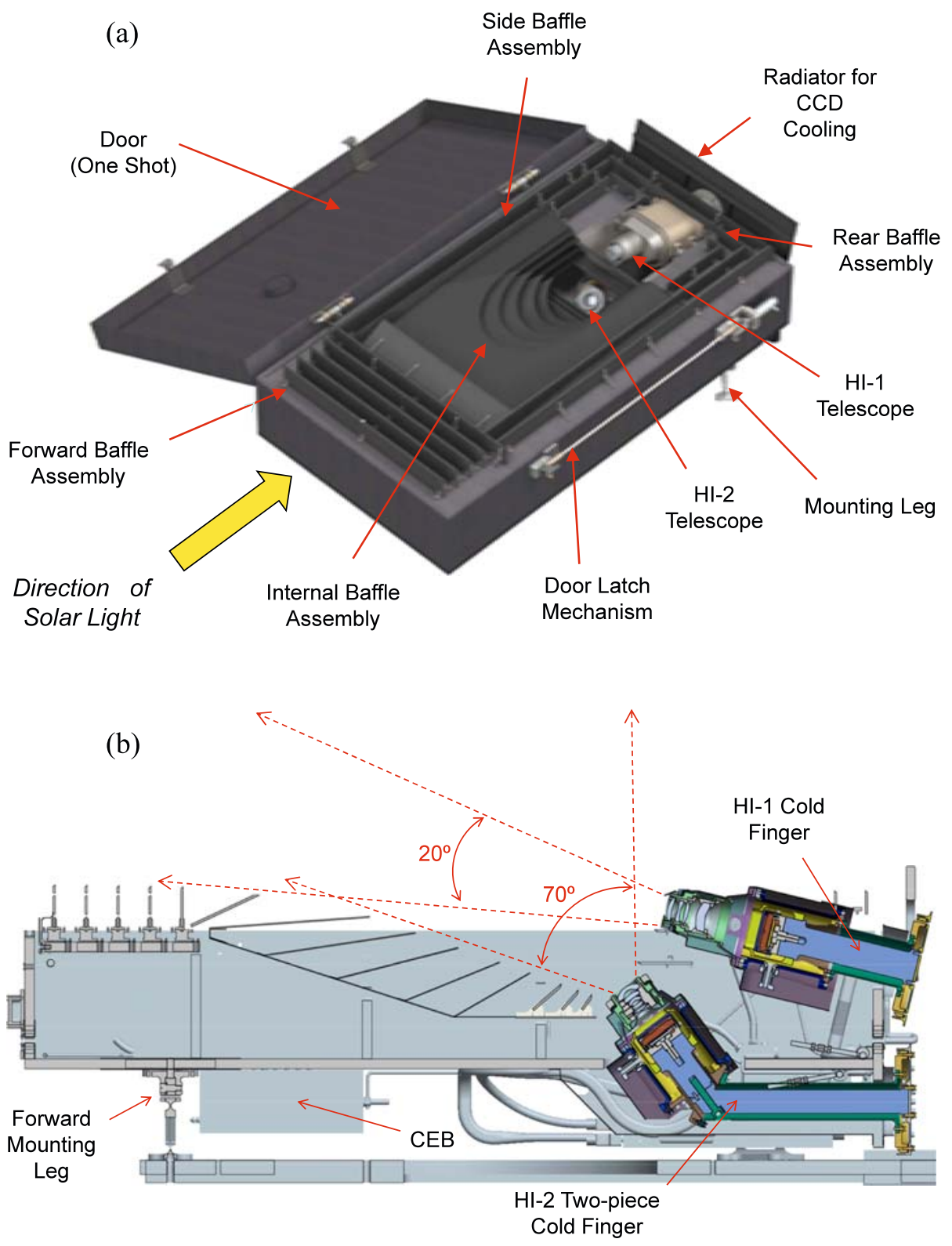

Figure 3 (a) The Heliospheric Imager design concept. (b) A cross-sectional view through the instrument, showing the fields of view of the two telescopes.

approximately 4.8 seconds, including overheads. Mainly because of mechanical accommodation constraints, the cameras do not have shutters, so the fact that the readout time is not insignificant compared with the exposure time results in the images being smeared during the readout process. However, as discussed in Section 10.1.2, this image smearing resulting 
Table 1 Performance specifications of the HI instruments.

\begin{tabular}{|c|c|c|}
\hline & HI-1 & HI-2 \\
\hline Direction of centre of field of view from Sun centre & $14.0^{\circ}$ & $53.7^{\circ}$ \\
\hline Angular field of view & $20^{\circ}$ & $70^{\circ}$ \\
\hline Angular range & $\begin{array}{l}4^{\circ}-24^{\circ} \\
\left(15 R_{\odot}-90 R_{\odot}\right)^{\mathrm{a}}\end{array}$ & $\begin{array}{l}18.7^{\circ}-88.7^{\circ} \\
\left(70 R_{\odot}-330 R_{\odot}\right)^{\mathrm{a}}\end{array}$ \\
\hline CCD pixel size & 35 arc-sec & 2 arc-min \\
\hline Image array $(2 \times 2$ binning $)$ & $1024 \times 1024$ & $1024 \times 1024$ \\
\hline Image bin size & 70 arc-sec & 4 arc-min \\
\hline Spectral bandpass & $630-730 \mathrm{~nm}$ & $400-1000 \mathrm{~nm}$ \\
\hline Exposure time $^{\mathrm{b}}$ & 40 seconds & 50 seconds \\
\hline Exposures per summed image sequence ${ }^{b}$ & 30 & 99 \\
\hline Summed image cadence ${ }^{b}$ & 40 minutes & 2 hours \\
\hline Brightness sensitivity $\left(B_{\odot}=\right.$ solar disc) & $3 \times 10^{-15} B_{\odot}$ & $3 \times 10^{-16} B_{\odot}$ \\
\hline Stray-light rejection (outer edge of field) & $3 \times 10^{-13} B_{\odot}$ & $10^{-14} B_{\odot}$ \\
\hline
\end{tabular}

${ }^{\text {a }}$ Measured in the plane of the sky relative to the viewing location.

${ }^{\mathrm{b}}$ These are actual values optimised during mission operations rather than original specifications (see Section 9.3).

from the shutterless operation of the cameras is correctable during subsequent data processing.

The geometrical layout of the fields of view of the HI telescopes, together with the COR2A and B fields, is shown in Figure 4(a). The HI-A telescopes have their optical axes pointed nominally in the ecliptic plane, at elongation angles to the east of the Sun, whilst HI-B axes are pointed to the west. The HI-1 and HI-2 fields provide an opening angle from the solar ecliptic of about $45^{\circ}$, chosen to match the typical size of a CME. There is significant overlap between the HI-1 and HI-2 fields of view, permitting photometric cross-calibration of the instruments. There is also continuous coverage from the COR-2 fields into the HI-1 fields, at least close to the ecliptic plane.

Although the optical designs are optimised for the circular fields of view of diameter $20^{\circ}$ and $70^{\circ}$, the square format of the CCD detectors results in some response in the regions indicated by dotted lines in Figure 4(a), although there is significant optical vignetting in these corners and, in the case of HI-2, the two corners on the side closest to the Sun are blocked by the internal baffles (see Figure 3).

The diagram serves to illustrate the scale of the imaging coverage of the mission, providing a view of the Sun - Earth line from the SECCHI coronagraph fields to the Earth and beyond. The Earth, as viewed from the different spacecraft, appears to move along the ecliptic plane during the mission, and during the second and subsequent years of science mission operations it will be in the fields of both HI-2A and HI-2B.

Although the configuration is shown in Figure 4(a) as a simple 2D diagram, it must be emphasised that the HI-A and HI-B viewpoints are from two widely separated spacecraft at similar planetary angles (Earth - Sun - spacecraft), thereby providing a stereographic view. Consequently, once the spacecraft are well separated, the HI-A and HI-B fields will cover common areas of the heliosphere. This is illustrated in Figure 4(b), which shows a projection onto the ecliptic plane of the overall fields of view of the HI instruments from the two spacecraft, on 1 October 2008. The region of overlap of the fields is indicated. 


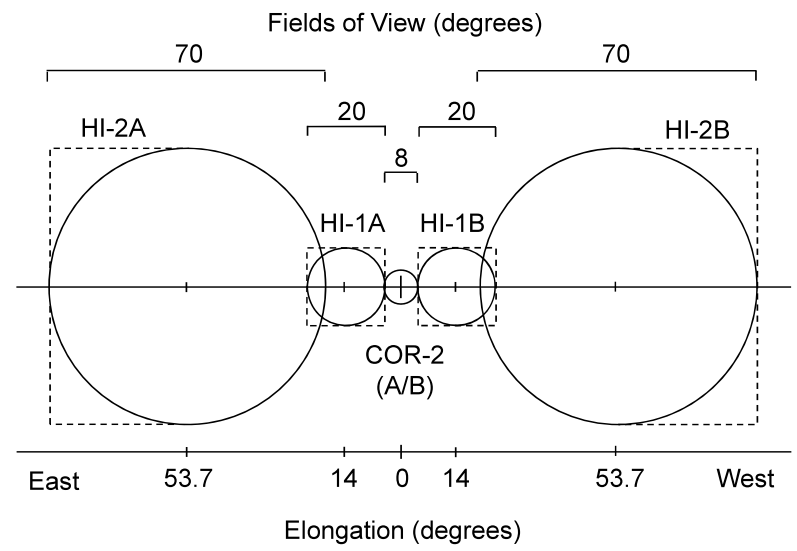

(a)

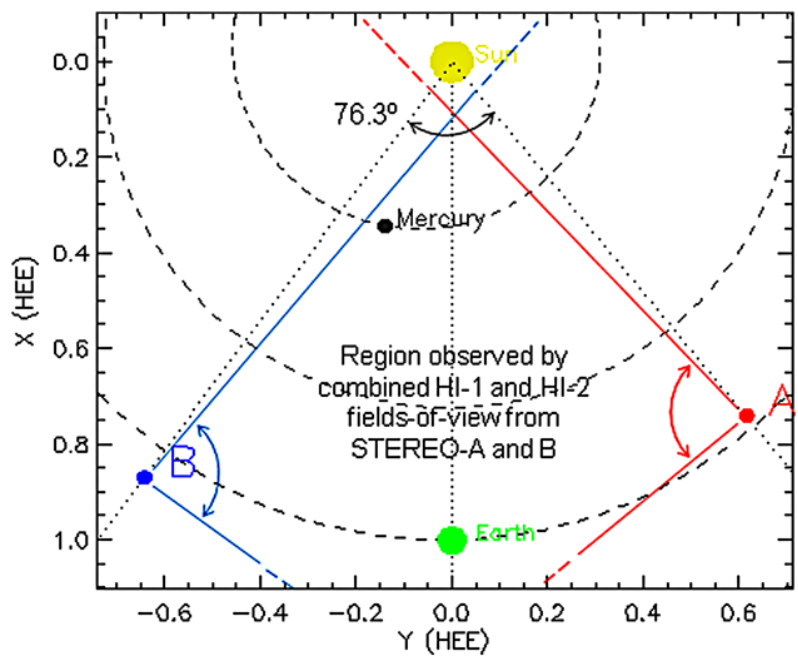

(b)

Figure 4 (a) The fields of view of the HI telescopes and the COR-2 Sun-centred coronagraphs. The dotted lines correspond to the square format of the CCD detectors, although the response in these corners is limited by optical vignetting. The Sun-centred coronagraphs view all solar latitudes, whilst the HI fields of view are limited to a maximum of $\pm 35^{\circ}$ perpendicular to the ecliptic. (b) A projection onto the ecliptic plane of the overall HI fields of view on 1 October 2008. The positions of the Sun, Earth, and Mercury are shown, together with the orbit of Venus (although Venus itself was not in the region plotted at this time). The spacecraft - Sun - Earth separation angles were $36^{\circ}$ and $39.8^{\circ}$ for STEREO-A and STEREO-B, respectively, giving a total separation angle of $76.3^{\circ}$. The coordinates are heliocentric Earth ecliptic Cartesian coordinates in units of $1 \mathrm{AU}$.

The positions of the two spacecraft as shown in Figure 4(b) are at distances from the Sun of 0.96 and 1.08 AU for STEREO-A and STEREO-B, respectively. During the course of the science mission the orbit of STEREO-A varies over the range of approximately $0.95-$ 0.97 AU with a period of about 1 year, whereas that of STEREO-B varies over the much larger range of $1.00-1.09 \mathrm{AU}$.

Figure 5, adapted from Socker et al. (2000), also shows the geometry of the HI fields of view but in addition shows the expected total intensity of the $\mathrm{K}$ - plus F-coronae as a 
Figure 5 The field-of-view geometry of the HI telescopes and the anticipated intensities of the corona and typical CMEs, adapted from Socker $e t$ al. (2000).

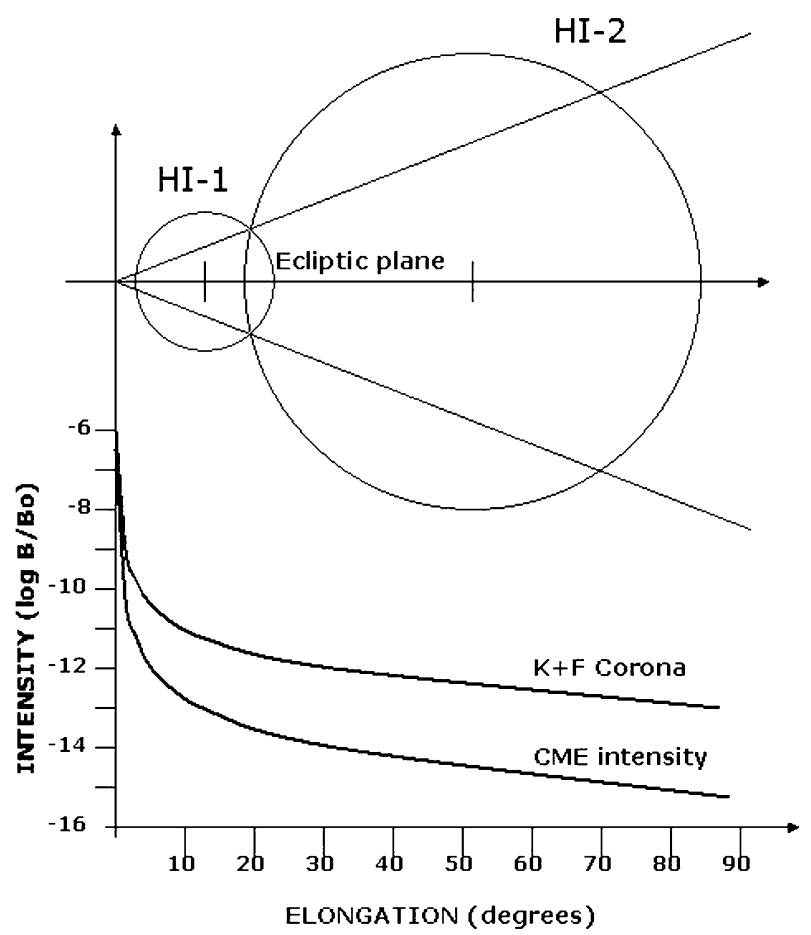

function of elongation angle, based on the calculations of Koutchmy and Lamy (1985), together with the typical CME intensity, both in units of $B_{\odot}$, the solar disc intensity. It is immediately evident that the total coronal intensity is about two orders of magnitude brighter than the anticipated CME signal and this defines the basic operational requirements for the instrument. As a consequence, one must accumulate for long durations so that the CME signal is larger than the statistical accuracy to which the background corona is measured, to extract the weak CME signal.

In order that the instrument stray-light level does not contribute significantly to the statistical error in measuring the coronal signal, it must be at least an order of magnitude lower, which can be seen from Figure 5 to require levels of better than $\sim 10^{-13} B_{\odot}$ for HI-1 and $\sim 10^{-14} B_{\odot}$ for $\mathrm{HI}-2$.

The brightness sensitivity requirements stated in Table 1 are based on the need to extract the CME signal from the other signal sources, which demands the detection of CME intensities down to $3 \times 10^{-15} B_{\odot}$ and $3 \times 10^{-16} B_{\odot}$, for the two telescopes. The complexity of the extraction of the CME signal from the raw data deserves further description, and this is addressed in detail in Section 10.

The principal hardware development for $\mathrm{HI}$ was centred at Birmingham University, UK, with camera design and development work and some thermal work provided by the Science and Technologies Research Council Rutherford Appleton Laboratory (RAL), UK. The Centre Spatial de Liège (CSL), Belgium, provided design of the optical and baffle systems, stray-light analysis and tests, and instrument calibration and qualification. Various aspects of the assembly, integration and test work, and the overall SECCHI management have been performed by the U.S. Naval Research Laboratory. The HI concept was developed by Dennis Socker of the Naval Research Laboratory. The operational and scientific lead for the HI instruments is provided through RAL. 

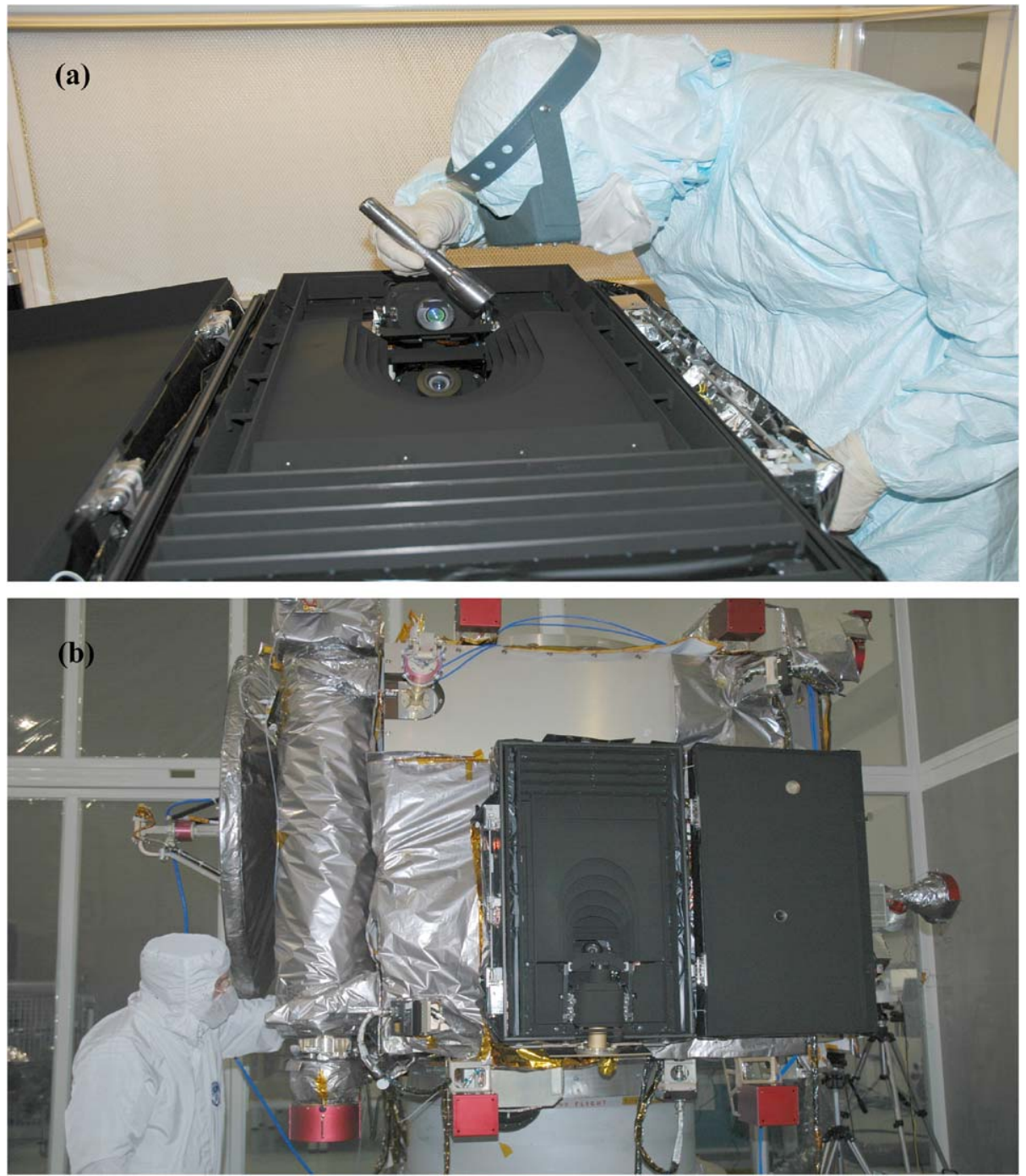

Figure 6 (a) HI-B undergoing final cleanliness inspection prior to delivery. (b) HI-A mounted on STEREO-A spacecraft at Astrotech Inc., Florida.

Figure 6 shows the HI-B instrument undergoing a final cleanliness inspection prior to delivery and the HI-A instrument after integration onto the STEREO-A spacecraft.

The HI instruments are operating successfully in orbit and Figure 7 shows an image obtained using the HI-1A camera with an example of a CME passing through the heliosphere. The subsequent sections of this paper describe the precise details of the HI instruments and their performance, and some more early results are given at the end. 
Figure 7 An image of a CME obtained using the HI-1 instrument aboard STEREO-A on 5 November 2007. The frame is $20^{\circ}$ across and the Sun is $4^{\circ}$ off the right-hand edge. The Milky Way is clearly visible in the lower left part of the image, with Jupiter in the centre of the image, close to the left-hand edge. The bright object in the southern extremity of the CME is Antares. Stars down to at least 12th magnitude are visible.

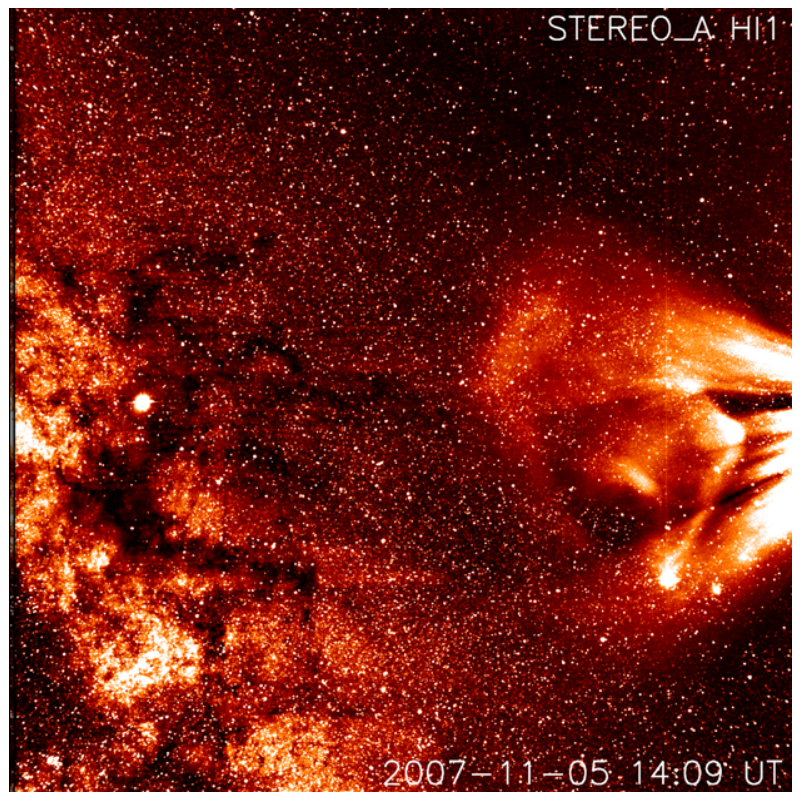

\section{Stray Light and Optical Design}

\subsection{Baffle Design}

The baffle design is the key to the HI concept. As shown in Figure 3, the baffle subsystems consist of a forward baffle, a perimeter baffle (side and rear baffles), and the internal baffle assembly.

The forward and perimeter baffles have been designed by theoretical modelling of diffraction systems (Defise et al., 2001), and the internal baffle by ray-tracing analysis, taking into account the various stray-light sources that HI will encounter, which include

- the Sun,

- the Earth, which appears at various positions in the fields of view of the telescopes during the mission (typically from $60^{\circ}$ to $90^{\circ}$ elongation direction during the main science phase),

- the bright planets and stars (e.g., Venus, Sirius, etc.),

- the zodiacal light or F-corona, according to the model of Koutchmy and Lamy (1985),

- one of the three SWAVES antennae, a component of another instrument aboard STEREO that is outside the HI-2 field of view in the antisunward direction but could conceivably scatter stray light into the HI instrument (see Figure 2 and Section 3.1.3), and

- any other spacecraft components that could potentially scatter stray light into the instrument.

Table 2 gives the order of magnitude of the intensity of these sources in units of $B_{\odot}$.

The basic function of the forward baffle is to reject the solar disc intensity, reducing solar stray light to the required levels. The perimeter baffle is primarily designed to reject stray light from the spacecraft, and the internal baffle system is designed to reject light from the Earth, planets, stars, F-corona, and the SWAVES antenna. 
Table 2 Stray-light source intensities (incident on $\mathrm{HI}$ instrument).

\begin{tabular}{ll}
\hline Source & Intensity $(B / B \odot)$ \\
\hline Sun & 1 \\
Earth & $10^{-6}$ to $10^{-9}$ \\
Planets (Venus) & $10^{-6}$ to $10^{-9}$ \\
Stars & $10^{-9}$ to $10^{-11}$ \\
Zodiacal light & $10^{-9}$ to $10^{-12}$ \\
SWAVES antenna & $10^{-5}$
\end{tabular}

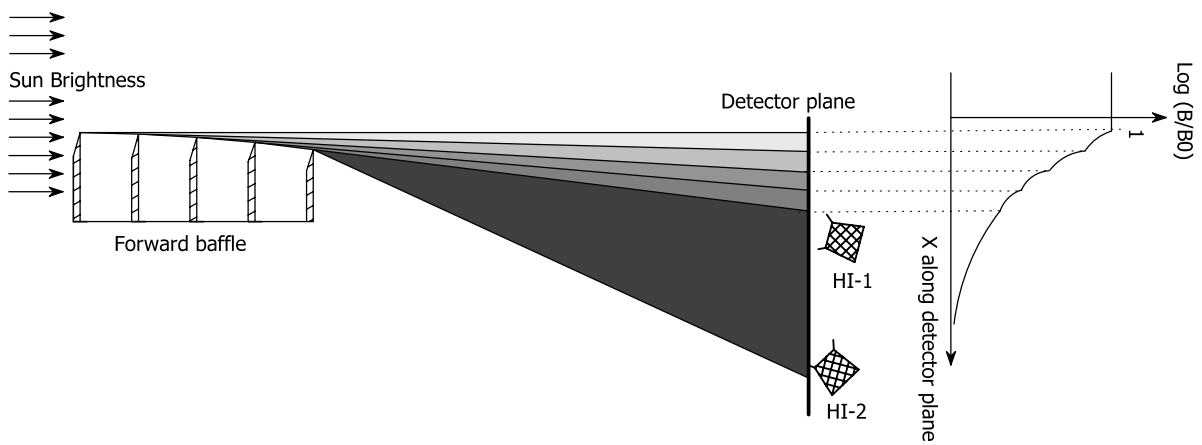

Figure 8 The concept of the cascade knife-edge diffraction system of the forward baffle, showing the typical locations of the entrance apertures of the HI-1 and HI-2 optics and the characteristic form of the expected rejection function.

\subsubsection{The Forward Baffle}

The forward baffle protects the HI-1 and HI-2 optical systems from direct solar light by means of a knife-edge cascade system. The concept of the baffle system is shown in Figure 8. The heights and separations of the five vanes are optimised to form an arc such that the $(n+1)$ th vane lies below the shadow line of the $(n-1)$ th and $n$th vanes. Figure 8 also shows typical locations of the entrance apertures of the HI-1 and HI-2 optics, both of which lie in the shadow region of the fifth vane. This five-vane system allows the required rejection to be achieved, as computed using Fresnel's second-order approximation of the FresnelKirchhoff diffraction integral for a semi-infinite half-screen (Defise et al., 2001).

Figure 9 shows the calculated rejection factor $\left(B / B_{\odot}\right)$, where $B_{\odot}$ is the solar brightness, plotted against distance below the horizontal shadow line cast by the first vane. The contributions of the successive vanes to the overall rejection curve are shown, and it can be seen that the rejection factors at the entrance apertures of HI- 1 and HI- 2 optics are $5 \times 10^{-11}$ to $8 \times 10^{-10}$ and $2 \times 10^{-12}$ to $3 \times 10^{-12}$, respectively. Since the baffle edges and the stray light diffracted from them are outside the fields of view of the optical systems, a further attenuation factor of at least $10^{-3}$ can be expected from the optics so that the overall predicted rejection of the direct solar flux easily meets the requirements for the outer edges of the fields of view given in Table 1. The experimental verification of the rejection factors using a prototype of the forward baffle system is described in Section 8.2. 
Figure 9 The calculated rejection factor of the forward baffles as a function of distance below the horizontal shadow line cast by the first vane, at nominal spacecraft attitude. The approximate locations of the entrance apertures of HI-1 and HI-2 optics are also shown.

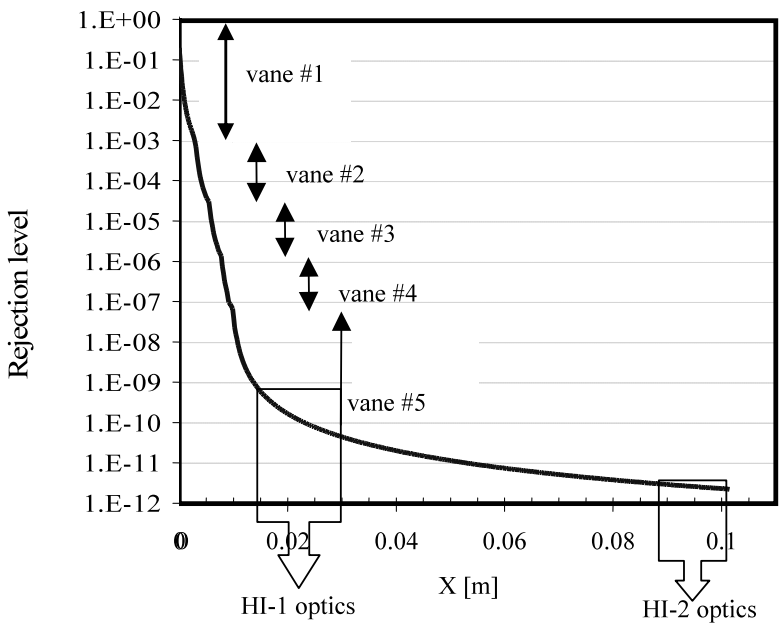

\subsubsection{The Internal Baffle}

The internal baffle system consists of a set of five principal vanes, complemented by a set of two small linear vanes located between the HI-1 and HI-2 entrance apertures, a set of three small linear vanes located under the HI-2 aperture, and a linear vane located just behind the forward baffles. The principal vanes have oval-shaped cutouts so that their edges lie just outside (nominally by $1^{\circ}-2^{\circ}$ ) the conical field of view of the HI- 2 optics (see Figure 3 ). The performance of this baffle system is determined by the location of the tip of each vane and the angles of the vanes relative to the optics field-of-view direction. The system attenuates the intensity of unwanted light (from stars, planets, the Earth, zodiacal light, and the SWAVES antenna) entering the HI-1 and HI-2 optical systems, by means of multiple reflections in the baffles. Although some of these objects may potentially be within the HI fields of view, the baffles limit the uniform background scattered into the optical systems from them.

The performance of the internal baffles was computed by using the Advanced Systems Analysis Package (ASAP) stray-light analysis software from Breault Research Organisation. Figure 10 shows a representation of the finite-element model used. The results indicated a rejection factor better than $10^{4}$ for every incident direction, with efficiency optimised for light sources coming from the rear of the instrument (in particular the SWAVES antenna).

\subsubsection{The Perimeter Baffle}

The perimeter baffle (side and rear baffles) is a three-sided rectangular system composed of two edges, similar to the forward baffle, that protects the HI optical systems from reflection of photospheric light by spacecraft components lying below the horizon plane defined by the baffles, including the low-gain antenna and the HI door mechanism.

It should be noted that one spacecraft component does rise above the plane of the perimeter baffles, namely one of the 6-m-long monopole antennae of the SWAVES instrument, although it is not within the field of view of either telescope (see Figure 2). As discussed previously, calculations show that scattered light from the monopole will be adequately trapped by the internal baffle system. 
Figure 10 Ray-tracing model of the internal baffle.

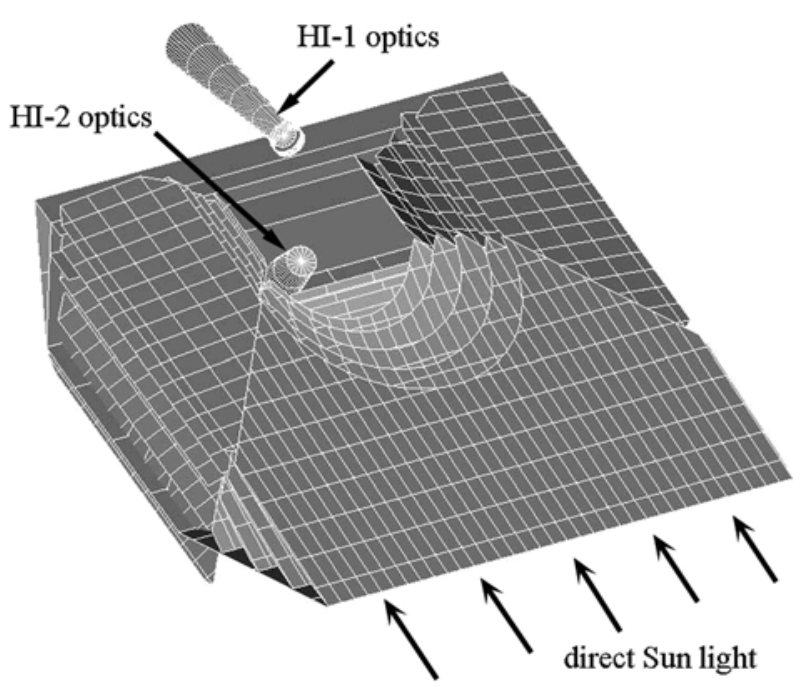

\subsubsection{Overall Baffle Rejection Factors}

Figure 11 shows the computed resultant diffuse background (in units of $B_{\odot}$ ) of the straylight sources at the HI-1 and HI-2 detectors, including attenuation by the forward and internal baffles, and the out-of-field rejection of the optics (assumed to be $10^{-3}$ ). These values do not include the contributions from the stray-light sources located in the optical fields of view (e.g., zodiacal light, Earth, and stars), which are directly imaged on the detectors but represent the contributions to the diffuse background from the various sources, which may be within or outside the field of view.

\subsection{Detailed Optics Design}

Figure 3 shows the locations of the HI-1 and HI-2 optics assemblies within the instrument. The optical configurations of the two assemblies are shown in Figure 12, which also indicates the Schott glass types selected for the various lens elements. (Glass types that have known radiation tolerance were selected, in particular for the space-facing elements.) The HI-1 lens system has a nominal focal length of $78 \mathrm{~mm}$ and aperture of $16 \mathrm{~mm}$, whilst the HI-2 system has a nominal 22-mm focal length and a 7-mm aperture. It should be noted that for multielement lenses such as these, the focal length does not correspond to the distance between the first element and the focal plane which is shown in Figure 12. In both cases the design is not diffraction limited and is thus optimised to minimise the rms (root mean square) spot diameter over an assumed operational temperature range of $-20^{\circ} \mathrm{C}$ to $+30^{\circ} \mathrm{C}$. The detector system at the focus in each case is a $2048 \times 2048$ pixel CCD with $13.5 \times 13.5 \mu \mathrm{m}$ square pixels.

The design of the HI-1 optics was mainly driven by the mechanical accommodation constraints on the first lens - the lens aperture itself must lie at the appropriate location in the shadow pattern of the forward baffle, as shown in Figures 8 and 9, whilst the lens barrel and the housing for the focal plane assembly of the camera must lie below the shadow line cast by the first baffle edge, so that direct sunlight cannot reach these components and thereby cause unacceptable back-scattering into the various baffle systems. The required spectral 
Figure 11 Diffuse background at (a) the HI-1 and (b) the HI-2 detectors, for the various stray-light contributions (in $B / B \odot$ units). (a)

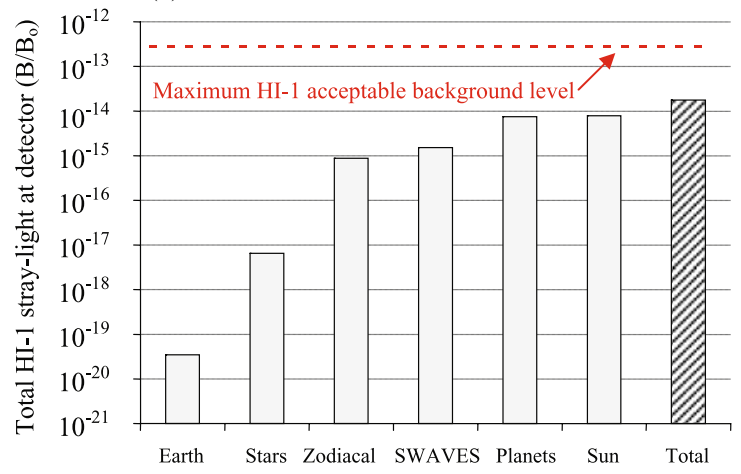

(b)

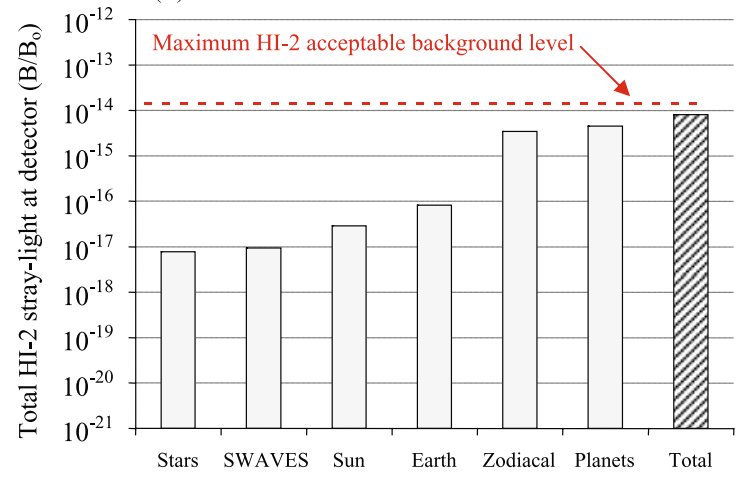

response of HI-1 $(630-730 \mathrm{~nm})$ is achieved by means of multilayer bandpass coatings on the two internal lens surfaces that have the minimum angles of incidence.

The HI-2 optics were designed to give the required large field of view $\left(70^{\circ}\right)$, together with wide spectral range and minimum contribution from image ghosts. These requirements made the design particularly challenging.

The lens systems are potential contributors to stray-light contamination, as a result of ghosts produced by multiple reflections from the optical surfaces and also reflections from the internal surfaces of the lens barrels. To minimise the intensities of the ghosts and also to maximise the throughput of the optics, standard antireflection (AR) coatings are used where possible on all lens surfaces in both assembles. The first surface, exposed to space, is coated with $\mathrm{SiO}_{2}$ to give good radiation tolerance as well as relatively robust handing characteristics. $\mathrm{MgF}_{2}$ coatings are generally used on the internal surfaces.

In the case of HI-2, the effectiveness of the AR coatings is limited by the large spectral range and the wide range of incidence angles. Consequently, the optics design was specifically optimised to spread the ghosts into larger regions at the focal plane. By doing so, the ghosting effects from bright objects within the field of view contribute to the overall diffuse background, rather than generating multiple images with spacing dependent on position in the field of view.

To reduce reflections and scattering, the design of the inner geometry of the lens barrels was optimised by ray-tracing analysis, and some cavities were added to act as light traps. The internal surfaces of the lens barrels and other mounting components were treated with 
(a)

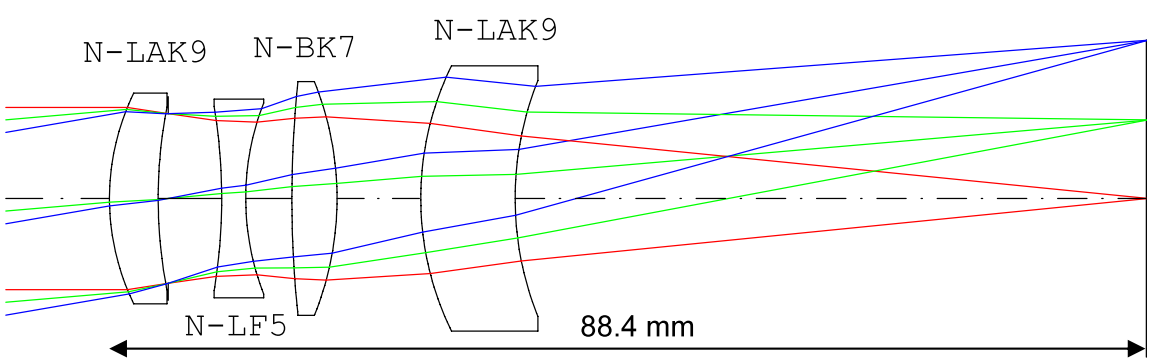

(b)

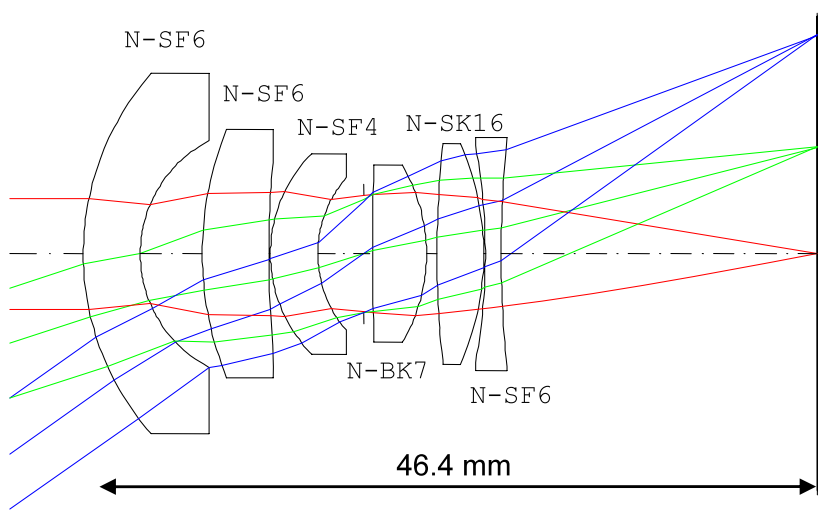

Figure 12 Optical configurations of (a) HI-1 and (b) HI-2 lens assemblies. The Schott glass types selected for the various elements are indicated beside each element.

either black copper oxide or black chromium coatings. These coatings provide additional absorption of those incident rays out of the field of view that are only partially suppressed by the aperture stop. A general attenuation factor of at least $10^{-3}$ was targeted for all the light outside the field of view and not suppressed by the aperture stop.

The design specifications and key performance parameters of the optical systems are summarised in Table 3. The results of performance tests on the optics units are described in Section 8.3.

\section{The Telescope Assemblies}

Each HI telescope assembly comprises the following major components:

- the lens assembly,

- the focal plane assembly (FPA), which contains the CCD detector, and

- the thermal radiator.

The HI-1 telescope assembly is shown in Figure 13. To show the internal construction, the protective covers and light seals over the lens barrel housing and FPA housing are not fitted. 
Table 3 HI-1 and HI-2 optical design parameters.

\begin{tabular}{lll}
\hline & HI-1 & HI-2 \\
\hline Field-of-view diameter & $20^{\circ}$ & $70^{\circ}$ \\
Focal length (nominal) & $78.46 \mathrm{~mm}$ & $21.67 \mathrm{~mm}$ \\
Optical axis length & $88.4 \mathrm{~mm}$ & $46.4 \mathrm{~mm}$ \\
$F$-number & $F / 4.93$ & $F / 3.08$ \\
Entrance pupil diameter & $15.9 \mathrm{~mm}$ & $7.0 \mathrm{~mm}$ \\
Spectral range & $630-730 \mathrm{~nm}$ & $400-1000 \mathrm{~nm}$ \\
Focal plane detector & $2048 \times 2048$ pixel CCD & $2048 \times 2048$ pixel CCD \\
& $13.5 \mu \mathrm{m}$ pixel pitch & $13.5 \mu \mathrm{m}$ pixel pitch \\
rms spot size & $14.9 \mu \mathrm{m}$ at $0^{\circ}$ & $40.6 \mu \mathrm{m}$ at $0^{\circ}$ \\
(against off-axis angle) $^{\mathrm{a}}$ & $16.5 \mu \mathrm{m}$ at $5^{\circ}$ & $46.0 \mu \mathrm{m}$ at $17.5^{\circ}$ \\
& $20.2 \mu \mathrm{m}$ at $10^{\circ}$ & $50.0 \mu \mathrm{m}$ at $35^{\circ}$ \\
\hline
\end{tabular}

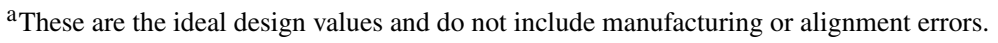

${ }^{b}$ Defined as the deviation from a linear projection at the edge of the (circular) field of view.

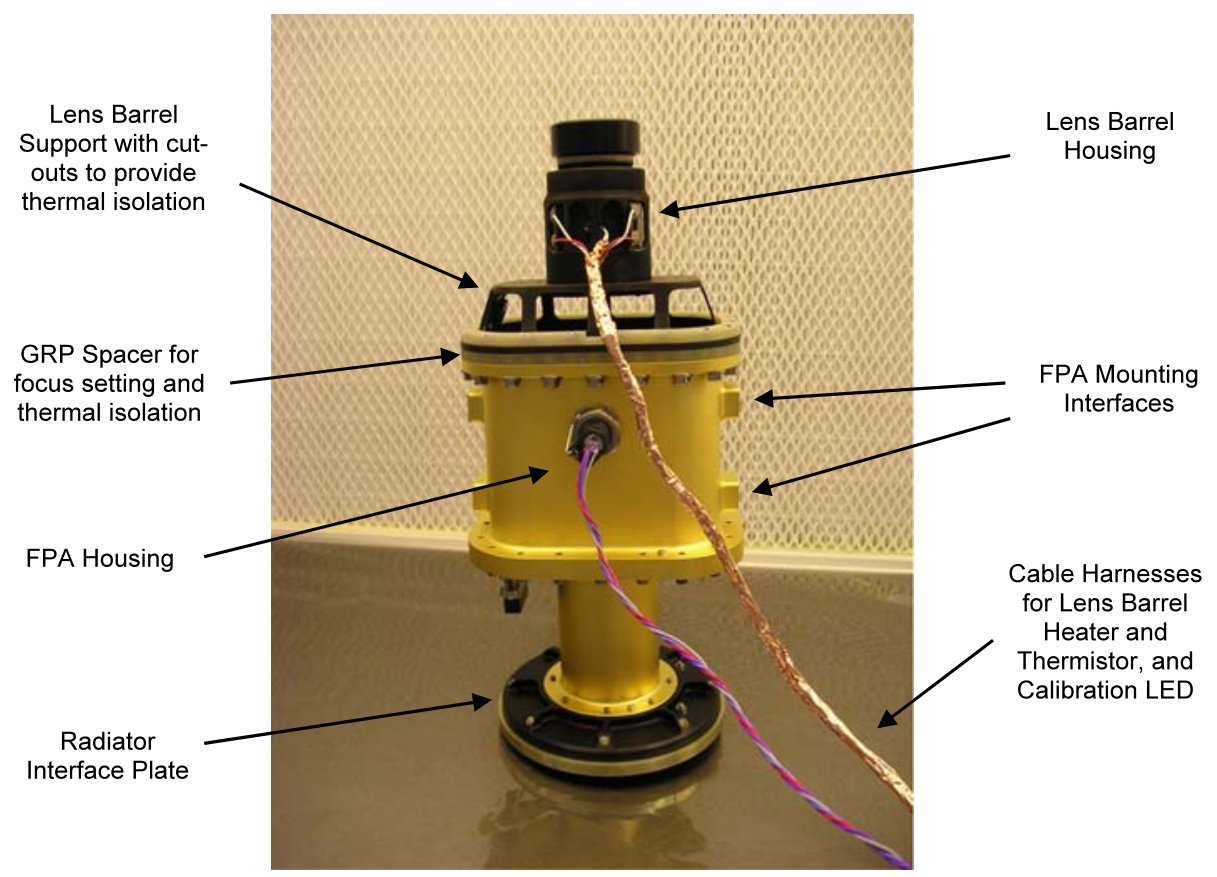

Figure 13 The HI-1 telescope assembly with the overall protective covers and light seals removed. The thermal radiator is not fitted. 


\subsection{The Lens Assembly}

The lens elements are mounted within a titanium lens barrel. Titanium was chosen to provide a good match to the coefficient of thermal expansion of the glass types used, thereby allowing a simple mounting arrangement using threaded rings to clamp the elements against shoulders on the barrel, without the need for compliant mounts. This mounting arrangement has been demonstrated to be acceptable over a survival temperature range of $-60^{\circ} \mathrm{C}$ to $+60^{\circ} \mathrm{C}$. The lens barrels were plated with a black copper oxide coating to reduce scattered light from out-of-field sources.

The lens barrels are fitted with heaters and thermistors to maintain the optics at appropriate temperatures in survival and operating cases. As shown in Figure 13, thermal isolation is provided between the warmer lens assembly and the cooler FPA and CCD detector assembly by means of cutouts in the titanium support structure and a glass reinforced plastic (GRP) spacer, which also allows the focus of the camera to be set.

A thin moulded carbon-fibre reinforced plastic (CFRP) cover is fitted over the lens barrel and FPA housings. This cover acts as a light seal and also as a thermal barrier to reduce thermal radiation from the warm lens assembly to space.

\subsection{The Focal Plane Assembly and Thermal Radiator}

The CCD detectors are mounted within the FPAs. Figure 14 shows a cutout view of the HI-1 FPA, with the lens assembly and the thermal radiator attached. The HI-2 FPA design is similar except that a two-piece construction with a clamped joint is used for the cold finger to satisfy the accommodation constraints within the instrument (see Figure 3(b)).

The main design drivers for the FPA assembly are to cool the CCD to the designed operating temperature whilst providing mechanical support. The location of the CCD relative to the focal plane of the optics must be accurately maintained over an operating temperature range extending from ambient down to the in-orbit operating temperature, and these alignments must still be maintained after the mission launch phase, with its associated vibration and quasistatic loads.

The CCD is cooled to around $-80^{\circ} \mathrm{C}$ by passive radiation from a thermal radiator that views deep space. The CCD is mounted in close thermal contact with the cold finger, which is manufactured from a high-conductivity aluminium alloy. Indium foil ensures good thermal contact between the cold finger and the radiator. The outer (i.e., space-facing) surface of the radiator is painted with a matte black paint, whilst the side that faces the back of the instrument structure is covered with low-emissivity thermal tape to reduce parasitic loads on the cooling system from radiative coupling with the warmer HI structure.

The FPA is supported within the HI structure by means of two mounting interfaces on each side (see Figures 13 and 14); these are also designed to enable the alignment of the telescopes relative to the structure. The main structural integrity of the FPA assembly is primarily provided by the FPA housing and the inner and outer support tubes. These components are manufactured from Ti6Al4V titanium alloy (an alloy containing approximately $90 \%$ titanium, $6 \%$ aluminium, and $4 \%$ vanadium). The use of this material, which has a thermal conductivity of only $7.2 \mathrm{~W} \mathrm{~m}^{-1} \mathrm{~K}^{-1}$, provides thermal isolation for the radiator/cold finger/CCD assembly. The external surfaces of the structural components are also gold-plated to reduce parasitic thermal loads from radiative coupling. In addition to its excellent thermal isolation properties, this titanium alloy has excellent mechanical properties (e.g., strength and stiffness), making it highly suitable for these structural components.

A black-anodised aluminium mask is located just in front of the CCD, with a cutout region matching the sensitive area of the $\mathrm{CCD}$. The function of this mask is to reduce the 


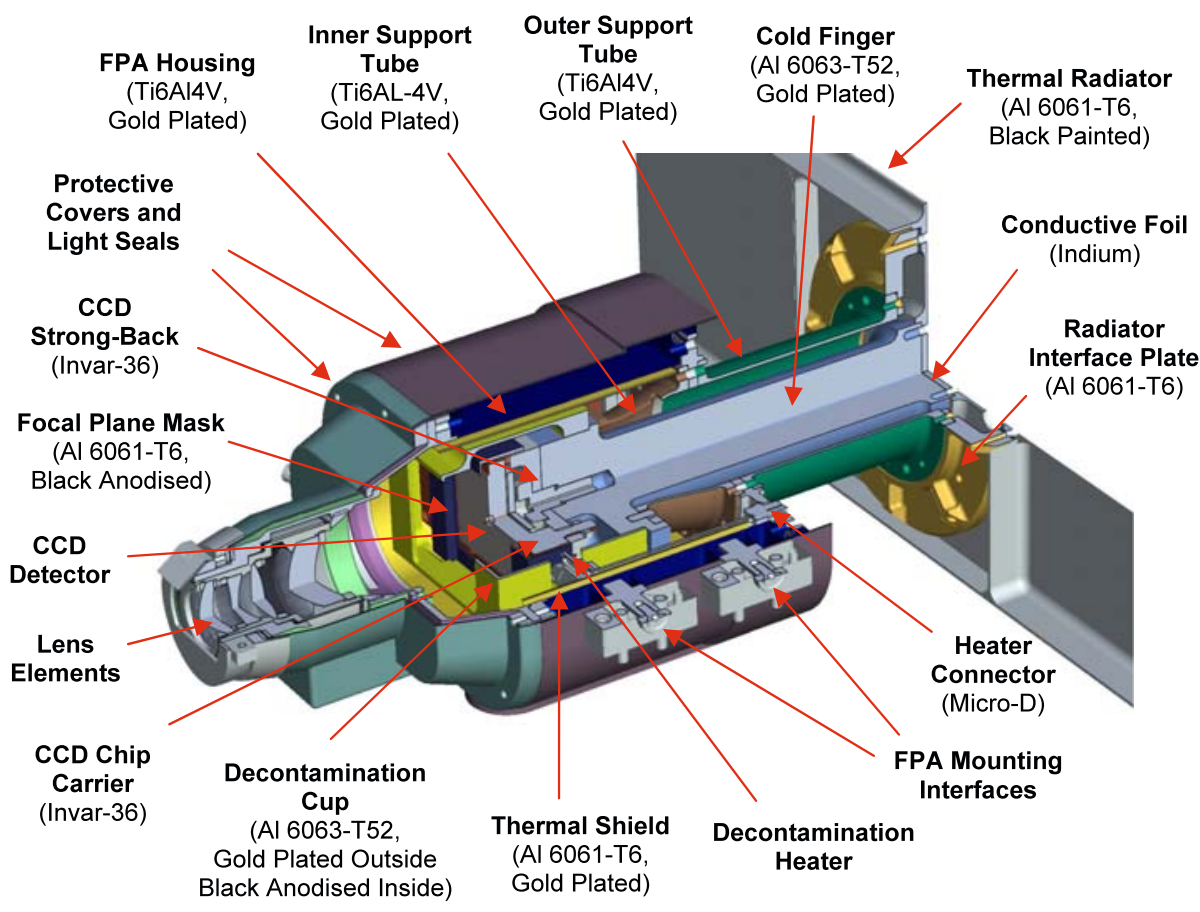

Figure 14 The HI-1 focal plane assembly with the lens assembly and thermal radiator attached.

possibility of stray light from the images of bright objects just outside the field of view from reaching the detector as a result of scattering off internal components within the FPA.

A heater mounted under the CCD chip carrier provides the capability of heating the CCD to at least $+30^{\circ} \mathrm{C}$ to drive off contaminants or to "anneal" the CCD after radiation damage. A cold cup (or decontamination cup), at a temperature slightly lower than the CCD, provides a contamination shield and a barrier against radiated heat loads. The internal surfaces of the cold cup are also black-anodised to inhibit the scattering of stray light.

The design satisfies the requirements to position the CCD at the focal plane of the telescope with the required accuracies perpendicular to and aligned with the optical axis. It also ensures that these alignments are maintained over a wide temperature range $\left(+20^{\circ} \mathrm{C}\right.$ to $-80^{\circ} \mathrm{C}$ ) and through the launch vibration environment.

\subsection{The CCD Detector}

All five telescopes within SECCHI use the same CCD architecture. The CCDs are e2v technologies (formerly Marconi Applied Technologies, also EEV) type CCD42-40 units, which are full-frame sensors with $2048 \times 2048$ pixels, each pixel being $13.5 \mu \mathrm{m}$ square (see Figure 15).

The CCDs are thinned, back-illuminated, and operate in a noninverted mode to ensure a full well capacity of more than 200000 electrons. The HI CCDs are coated with a standard AR coating optimised for the $450-750 \mathrm{~nm}$ spectral range (with the effectiveness of the coating degrading somewhat near the edges of the overall $400-1000 \mathrm{~nm}$ spectral range of HI-2). The quantum efficiency reaches $93 \%$ at around $550 \mathrm{~nm}$, but it drops to $23 \%$ and $30 \%$ 
Figure 15 The SECCHI EUVI

CCD in its carrier, with the

flexible printed circuit harness attached. The overall size of the CCD chip is about

$28.1 \times 30.3 \mathrm{~mm}$. The CCDs used in the $\mathrm{HI}$ instruments differ only in the presence of an AR coating.

Figure 16 The quantum efficiency of the HI CCDs as a function of photon wavelength. The filled points on the graph are typical experimental results obtained by e $2 \mathrm{v}$.
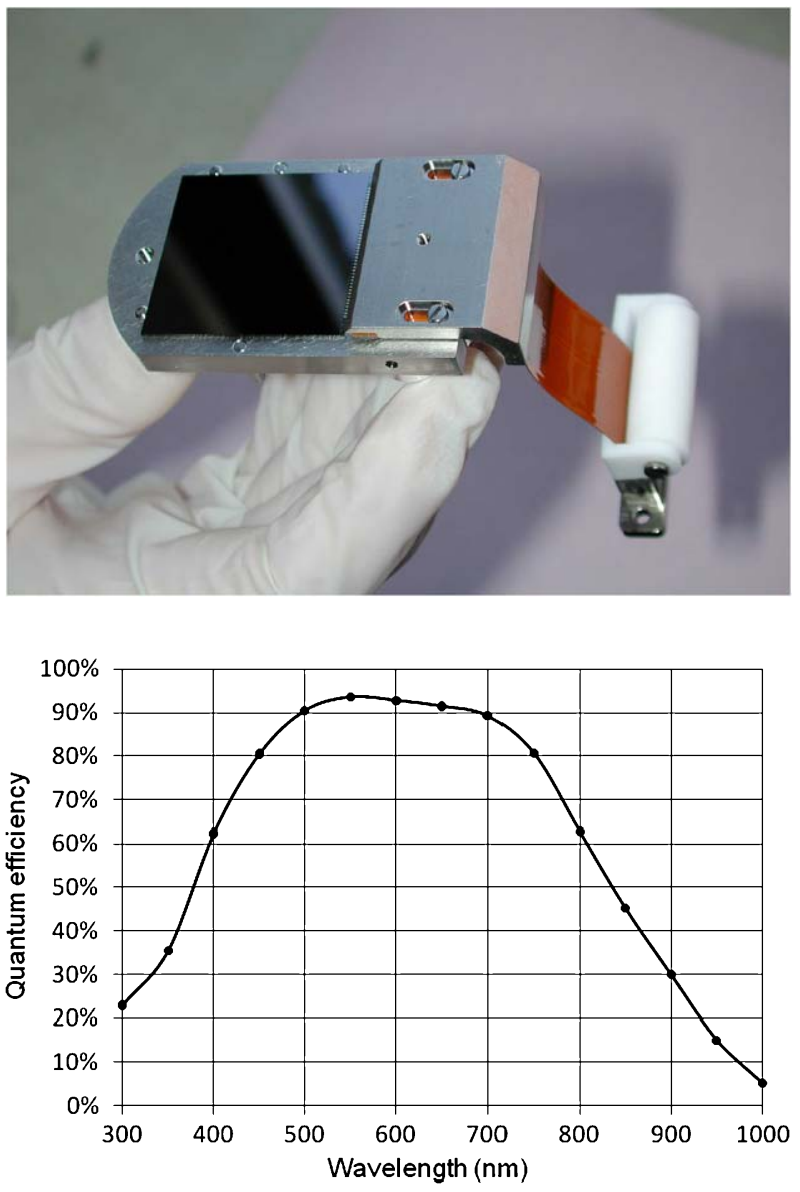

at 300 and $900 \mathrm{~nm}$, respectively (see Figure 16). The charge transfer efficiency is typically $99.9995 \%$ and the dark current at the $-80^{\circ} \mathrm{C}$ operating temperature is essentially negligible at $\sim 0.002 \mathrm{e}^{-}$pixel $^{-1} \mathrm{~s}^{-1}$ (see Section 9.1.1).

The CCD architecture has a single serial readout register with an output node at each end, although only one output node is used in the flight configuration to reduce the electronics complexity and mass. The register has 50 nonimaging or dummy locations at each end. These do not correspond to pixels in the CCD imaging region and must be shifted out before reaching the image data. They provide a measurement of the electronics offset or bias.

\section{Electronic Design and Instrument Data Handling}

\subsection{The Camera Electronics Box}

The CCDs in the HI cameras are read out by a camera electronics box (CEB). A similar CEB is used to read out the CCDs in the COR1, COR2, and EUVI instruments in the SECCHI suite. A more detailed description of the SECCHI CEB design is given in Waltham and Eyles (2007). 


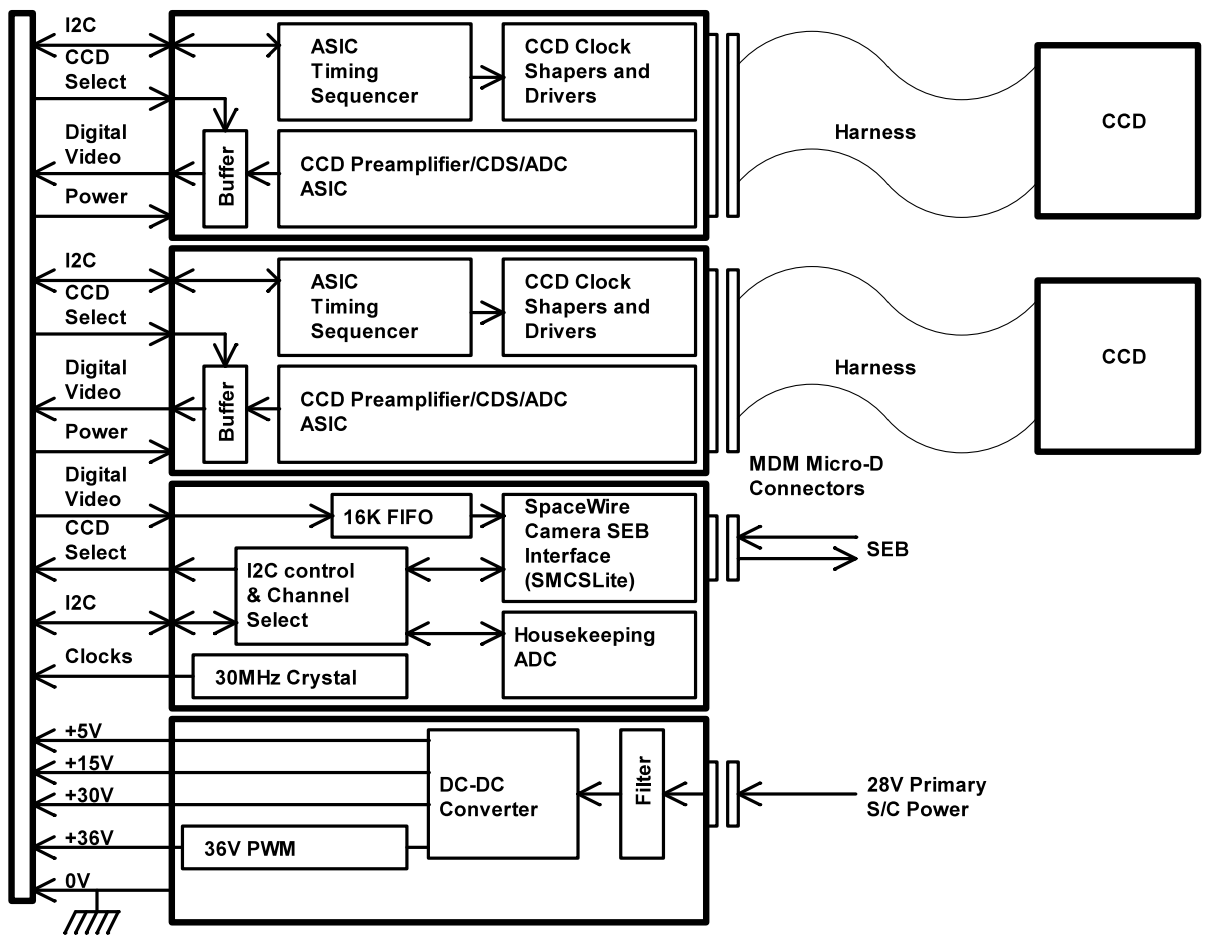

Figure 17 Block diagram of the HI CEB.

The HI CEB is accommodated within a housing of dimensions $195 \times 126 \times 70 \mathrm{~mm}$. The aluminium wall thickness is $2.0 \mathrm{~mm}$, and the mass is $1.45 \mathrm{~kg}$.

The design of the CEB is tailored to the requirements of the CCD42-40 and includes a requirement for a pixel readout rate of $1 \mathrm{Mpixel} \mathrm{s}^{-1}$. Very challenging low mass and power budgets dictated the use of application-specific integrated circuit (ASIC) and surface-mount packaging technologies to minimise the size, mass, and power requirements.

A block diagram of the HI CEB is shown in Figure 17. It comprises the following:

- two CCD driver and video processing cards, one dedicated to each of the HI CCD cameras,

- a camera interface card that provides a common interface between the two CCD driver cards and the SECCHI electronics box (SEB),

- a DC to DC power converter mounted in a separate internal screened housing in the base of the enclosure, and

- a backplane interface for interconnection of the daughter printed circuit boards.

Each CCD is controlled by a dedicated purpose-designed waveform generator and sequencer (WGS) ASIC (French et al., 1998). The logic-level clocks are then translated into high current-drive waveforms of appropriate shape and amplitude to drive the parallel and serial register electrodes of the CCD. The video output signal from the CCD is buffered through JFET transistors located within the FPA and passed to the CEB through a harness approximately $0.6 \mathrm{~m}$ in length. The video processing chain consists of a differential preamplifier, a correlated double sampler (CDS), and a 14-bit analogue-to-digital converter (ADC), 
all implemented within a second purpose-designed and radiation-tolerant ASIC (Waltham and Eyles, 2007). In addition, each CCD driver card contains low-noise DC bias supply generators for the CCD.

The CEB communicates with the SEB via an IEEE1355 SpaceWire link, enabling camera waveform programming, camera commanding, collection of telemetry housekeeping data, and the transmission of digitised CCD video data. The data transmission rate is $100 \mathrm{Mbits} \mathrm{s}^{-1}$.

Exposure timing for each CCD is controlled directly from the SEB. Appropriate programming of the WGS ASIC enables the CCD clock waveform patterns and tables to be set up or updated. Commands are then sent to the ASIC to initiate the various camera modes, specifically $i$ ) clearing of residual charge, $i i$ ) image exposure, and iii) full-frame, windowed, or continuous readout. On-chip image binning can be implemented by means of appropriate clock waveforms. The programmable video gain and DC offset level can be set up by means of commands sent to the CDS/ADC ASIC.

Housekeeping telemetry from the camera electronics enables the CEB secondary power supply voltage rails and the internal temperature to be monitored. The CCD temperatures are monitored directly from the SEB and not through the CEB. Similarly, the control of the decontamination heater power to the CCD is via the SEB.

The CEB contains a DC to DC power converter that converts the incoming $28-\mathrm{V}$ spacecraft primary power to the required $+5-,+15-,+30-$, and $+36-\mathrm{V}$ secondary supplies. The primary converter is an International Rectifier ART2815T, configured to provide $+5,+15$, and $+30 \mathrm{~V}$. An additional PWM (pulse width modulator) circuit boosts the $+30-\mathrm{V}$ secondary supply to the $+36 \mathrm{~V}$ required for the output transistor bias supply circuitry of the CCD42-40. The ART2815T is synchronised to a $150-\mathrm{kHz}$ clock derived from the $30-\mathrm{MHz}$ CEB master clock, thus satisfying a spacecraft requirement that the converter is synchronised to some multiple of $50 \mathrm{kHz}$.

The 28-V power supply input is in-rush current limited and current-trip protected. Electromagnetic compatibility filtering is provided by an International Rectifier ARF461 filter module and additional prefilter circuitry. The converter's output supply rails are also filtered to minimise any noise or current-switching transitions on the supply rails to the CCD drive circuitry. The complete power converter system is screened from the CCD drive electronics within a separate shielded compartment in the base of the CEB mechanical housing.

The CEB has the following operating modes:

- Clear: Clearing of residual charge from the CCD takes advantage of the CCD's "dumpdrain" running adjacent to the serial output register. Appropriate programming of the WGS ASIC allows the entire array to be cleared any number of times prior to integration.

- Integration: During integration, the CCD parallel register clocks are held at the static voltage levels required to set up the potential wells that define the pixels. The serial register clocks are also held static since the CCD is not being read out (or cleared) during this time.

- Readout: Appropriate programming of the WGS ASIC allows various readout modes, including:

○ full-frame readout of $n$ lines, each of $m$ pixels,

o windowed readout of at least two windows on the CCD,

$\circ$ full-frame or windowed readout with on-chip pixel binning, and

$\circ$ continuous clocking.

In the case of HI, the normal CCD readout format is to read out 2048 lines, each of 2176 pixels. Each line comprises 50 nonimaging or underscan pixels, 2048 imaging pixels, followed by 78 overscan pixels. The underscan pixels are useful for determining the DC offset 


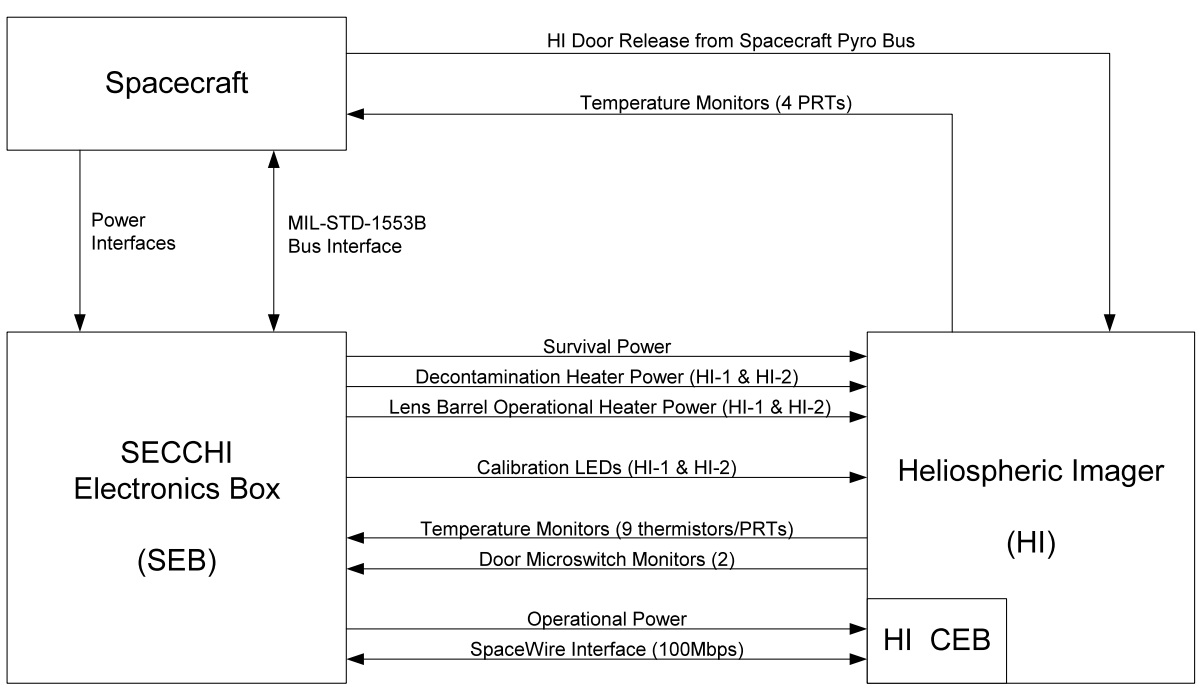

Figure 18 Electrical interfaces among the SECCHI electronic box, the spacecraft, and the HI instrument.

of the images. The overscan pixels ensure that the charge is completely cleared before the next line is transferred into the serial readout register.

Occasionally, other readout modes are used, including a $2176 \times 2112$ pixel readout, which provides 64 rows of vertical overscan.

Each pixel is read out as a 14-bit digital value. The overall system gain is approximately $15 \mathrm{e}^{-}$per ADU (analogue to digital unit) and the readout noise is typically $15 \mathrm{e}^{-} \mathrm{rms}$.

\subsection{Instrument Control and Monitoring}

The HI instrument does not have its own dedicated command and data handling system but instead is controlled by the SEB in common with all the other SECCHI instruments. The SEB is based around a RAD750 processor (from BAE Systems) and provides the instrument's main interfaces with the spacecraft via a MIL-STD-1553B bus, together with power interfaces. The SEB comprises four printed circuit boards - the RAD750 processor board, the MIL-STD-1553B interface board, the SpaceWire interface board, and the housekeeping board - together with power control and monitoring circuitry. Figure 18 shows a block diagram of the interfaces among HI, the SEB, and the spacecraft.

As described in Section 5.1, the SEB controls the CEB via an IEEE1355 SpaceWire link, including commanding of the camera exposures and reading out of the images. The onboard image processing is described in Section 5.3.

The SEB provides a total of six switched primary power rails to HI (nominally $28 \mathrm{~V}$; typically $32 \mathrm{~V}$ ) as follows:

- a survival heater bus to power survival heaters on the CEB and the lens barrels,

- an operational power bus for the CEB,

- operational power for the HI-1 and HI-2 lens barrel operational heaters, and

- a decontamination heater bus to power decontamination heaters mounted close to the CCDs in the HI-1 and HI-2 FPA assemblies. 
There are a total of nine SEB temperature monitors allocated to HI, which are either YSI44901 thermistors or PT100 platinum resistance thermometers (PRTs) depending on the required temperature range, in the following locations:

- PRTs mounted on the HI-1 and HI-2 CCDs,

- thermistors mounted on the HI-1 and HI-2 lens barrels, and

- five representative structure temperatures, including the CEB housing.

The thermistors on the lens barrels are used to control the duty cycle of the corresponding lens barrel operational heater, by means of a proportional-integral-differential control algorithm implemented in the SEB software. A control accuracy of better than $1^{\circ} \mathrm{C}$ is achieved.

The status of the HI door is monitored by two microswitches. One indicates that the door latch mechanism has been released and the other indicates that the door has reached the fully open position.

The SEB also operates calibration LEDs that provide an optical stimulus for the CCDs. The LEDs are mounted in the FPA assemblies so that light is scattered via indirect paths onto the CCD and they are pulsed with a programmable number of $20-\mu$ s pulses. Although the intensity at the CCD is not very uniform (varying by factors $\sim 3$ and $\sim 10$ for HI- 1 and HI-2, respectively) the LEDs are nevertheless useful for functionality testing, linearity checks, and determining small-scale (pixel-to-pixel) response variability.

The direct electrical interfaces between the $\mathrm{HI}$ and the spacecraft are:

- four PRT temperature monitors to provide an indication of the thermal status of the instrument even when the SEB is powered off and

- switched power (nominally $28 \mathrm{~V}$ ) from the spacecraft pyro bus to operate the wax actuator and release the HI door; two redundant circuits are provided.

\subsection{Onboard Data Handling and Image Processing}

To obtain the required signal-to-noise ratio, the HI images require much longer exposure times than the other SECCHI instruments, typically 20 minutes for HI-1 and more than 1 hour for HI-2. However, to avoid buildup of cosmic-ray hits (typically 45 pixels s$^{-1}$ for each CCD) and also saturation of the brighter regions of the image scene owing to the finite dynamic range of the $\mathrm{CCD}$, the total exposure time is made up by summing many much shorter exposures.

The SEB onboard software performs a number of image-processing operations on the individual exposures before sending the data to the spacecraft solid state recorder for storage and subsequent telemetry down-link. For normal science observations these are as follows:

- The individual exposures are scrubbed to remove cosmic-ray events. The algorithm used compares each new image with the previous image on a pixel-by-pixel basis - if the signal in a given pixel exceeds that in the previous image by more than a defined threshold, then the value for that pixel is replaced by the previous value. The threshold normally used is $5 \sigma$, where $\sigma$ is the predicted noise standard deviation based on the number of photoelectrons detected.

- A column of the underscan region of the readout is used to determine the DC offset of the image, which is then subtracted from all pixels.

- The image underscan and overscan regions are trimmed off and the resultant $2048 \times 2048$ image pixels are $2 \times 2$ binned.

- The resultant $1024 \times 1024$ binned 16-bit images are then summed over a number of exposures. To prevent integer overflow, the summed image is stored as a "low word" and a 
"high word" image, both 16 bits although only a few bits are significant in the high word image.

- For diagnostic purposes, including monitoring the rate of cosmic-ray hits, the values in the final bins of the last row of the summed image (in the direction of CCD readout) are overwritten with the numbers of pixels replaced by the cosmic-ray scrubbing algorithm in each individual exposure.

- The two-part images are then separately compressed by using a Rice lossless compression algorithm before down-link to ground. An overall compression factor of about 1.5 is achieved.

- The two-part images are reconstructed into $1024 \times 1024$ 32-bit images on the ground. Occasional telemetry errors (e.g., missing blocks) may affect either part of the downlinked image.

The details of the exposure sequences (e.g., individual exposure times, exposure cadences, and number of exposures summed) were refined during the instrument commissioning phase early in the mission and are described in Section 9.3. A total of 36 summed images for HI-1 and 12 for HI-2 are taken every 24 hours.

In addition to this image processing for HI science operations, the SEB software also permits other types of images to be down-linked for calibration and engineering purposes, including $i$ ) single full-resolution CCD exposures with $2048 \times 2048$ pixels, ii) single $2 \times 2$ binned exposures, and iii) exposures that still include the underscan and overscan regions. The SEB also produces more highly compressed and binned versions of the summed images; these are down-linked in real time via the STEREO Space Weather Beacon (see Section 9.4).

\section{Mechanical Design and Manufacture}

\subsection{The Main Structure}

The challenging mass allocation available to $\mathrm{HI}$, together with the stringent requirements to maintain stable relative alignments of various critical components, such as the forward baffles and the FPAs, over the very large temperature excursions experienced by the instrument between the ambient case and operational conditions in orbit, dictated the extensive use of CFRP and composites in the construction of the instrument. All the CRFP composites used were manufactured and assembled in the space-qualified composites fabrication facility at Birmingham University.

The main structure is a five-sided box, with internal stiffening bulkheads, shown conceptually in Figure 19. The structure was manufactured from CFRP composite panels, consisting of CFRP skins bonded onto both sides of 8-mm-thick aluminium honeycomb panel. The CRFP skins were manufactured from M55 unidirectional carbon fabric impregnated with RS3 cyanate ester resin (YLA Inc.). The M55/RS3 system has been used extensively in the aerospace industry on account of its impressive stiffness-to-mass ratios, together with its excellent dimensional stability and acceptably low outgassing properties.

The CFRP skins were fabricated by using a balanced "quasi-isotropic" layup with fibre orientations of $0^{\circ},+60^{\circ}$, and $-60^{\circ}$ for the different fabric layers. After laying up the fabric, the impregnated RS3 adhesive was cured under high temperature and pressure in an autoclave to produce the finished skin. A pair of such skins were then placed either side of a honeycomb panel, with film adhesive between the skins and the panel, and the whole assembly was again cured in the autoclave to form the composite panel. This type of construction has high strength and stiffness, together with low mass, and is very stable over a wide temperature range with a typical coefficient of thermal expansion $\approx 0.5 \times 10^{-6 \circ} \mathrm{C}^{-1}$. 


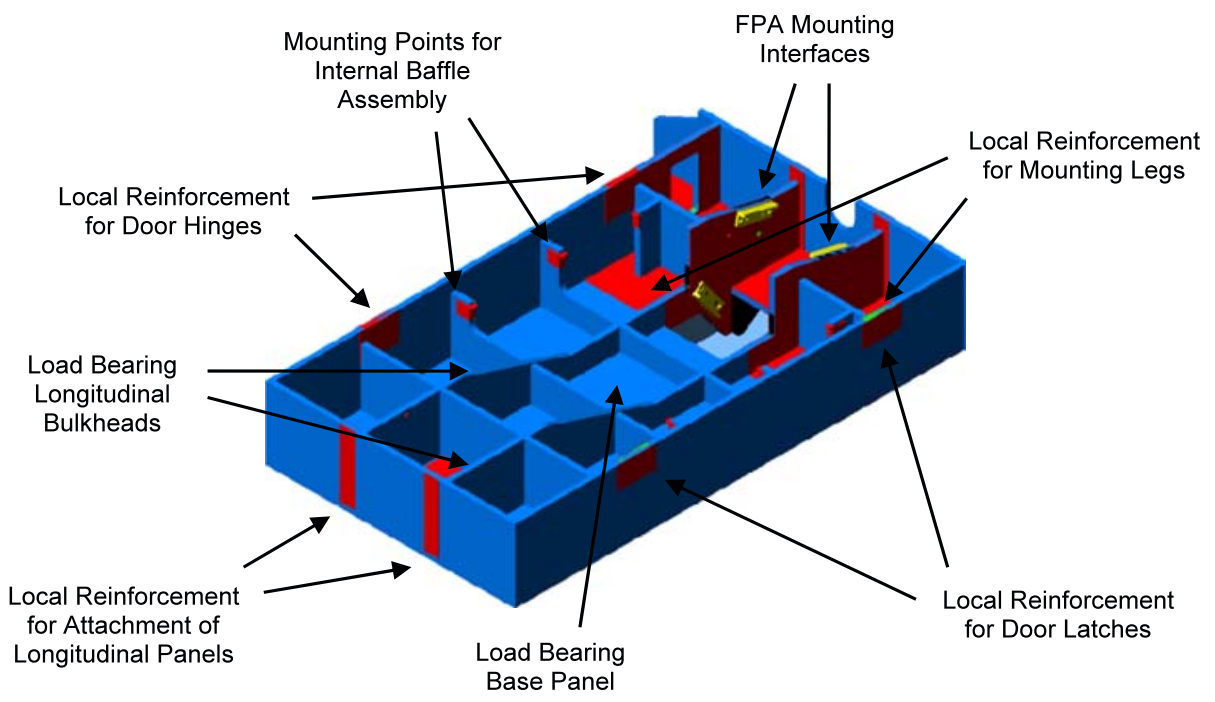

Figure 19 The concept of the HI main structure. Regions where the structure was reinforced by bonding extra thicknesses of CFRP skin material on the outside of the panels are indicated in red.

The CFRP panels were then machined to the dimensions required for the various component parts of the structure. The major load-bearing components (i.e., the base panel, the longitudinal bulkheads, and the four outer side, front, and back panels) were joined together by screwing through clearance holes into threaded inserts in solid CFRP blocks that had been bonded into the edges of the panels. In addition, all panels were bonded together with strips of L-shaped CFRP at all right-angle junctions. As indicated in Figure 19, load-bearing regions of the structure were reinforced locally by bonding an additional thickness of the CFRP skin material to the outer skin of the panel in the regions of high stress.

Considerable care was taken to ensure the cleanliness of the finished CFRP structure, both in terms of particulate and volatile contaminants. All materials, and in particular adhesives, used in the construction were selected for low outgassing properties (generally $<0.1 \%$ total mass loss and $<0.01 \%$ collected volatile condensable material). The exposed edges of all machined panels were capped, either by bonding a strip of U-shaped CFRP between the skins or by covering the exposed edges with black kapton tape. Venting to space was provided by regular patterns of small holes on the external faces of the outer panels. The aluminium honeycomb itself was perforated with small holes to permit venting from the internal panels into the outer panels and hence to space. Finally the entire assemblies were subjected to thermal vacuum bakeouts, monitored by temperature-controlled quartz crystal monitors (TQCMs), both at the level of the completed structure prior to installing the various subsystems and also during instrument-level assembly and testing.

The main structure is attached to the spacecraft by three titanium alloy (Ti6Al4V) mounting legs, each of which is designed to have some flexibility about one degree of rotational freedom. They are arranged so that together they form an approximation to a semikinematic mount, thereby minimising the loads that can be transmitted between the structure and the spacecraft as a result of differential thermal expansion (see Figure 20). The low thermal conductivity of the titanium alloy used also provides the required thermal isolation between the instrument and the spacecraft.

The major subsystems supported by the structure are the following: 


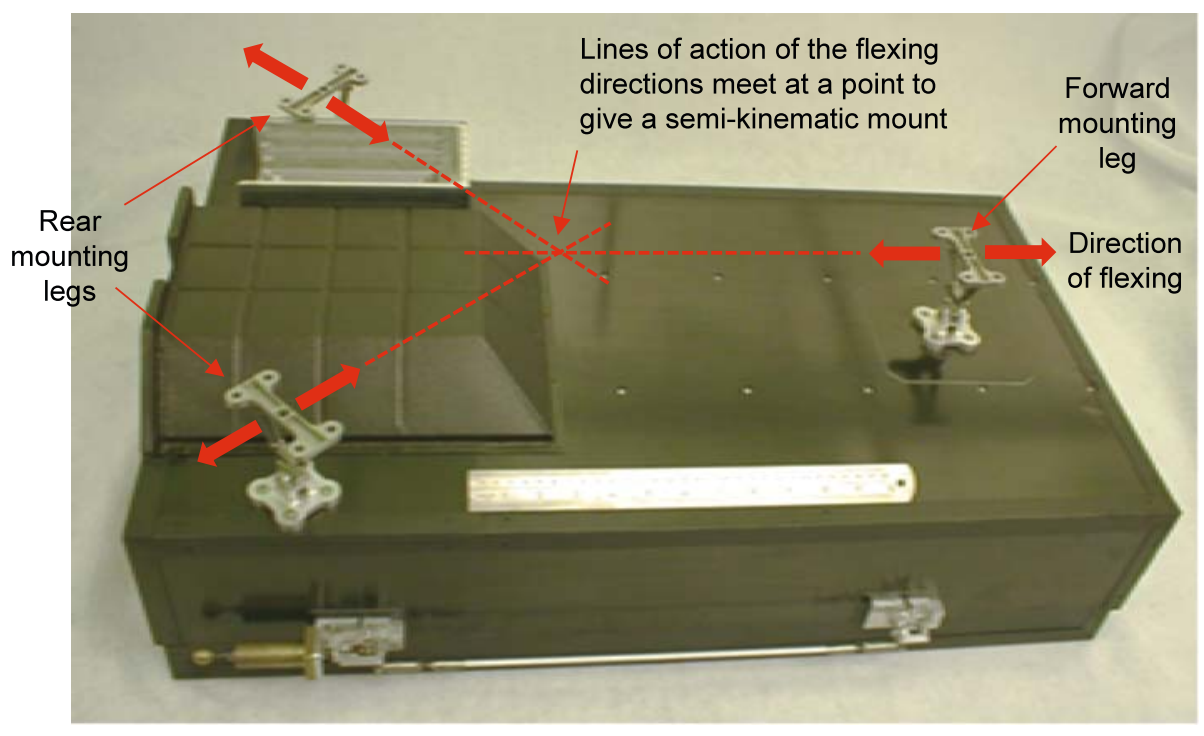

Figure 20 The underside of the qualification model of the HI main structure showing the configuration of the titanium mounting legs.

- the forward and perimeter baffles support panel,

- the internal baffle assembly,

- the focal plane assemblies,

- the door, including the latch mechanism, and

- the CEB, mounted on the underside of the base panel.

The entire HI main structure, baffles assemblies, and FPA housing covers were all painted with matte black paint (Aeroglaze Z307) to attenuate scattered light and achieve the required overall stray-light rejection.

The final mass of the $\mathrm{HI}$ instrument, with all components and subsystems installed but excluding the thermal blankets, was $15.05 \mathrm{~kg}$ (16.3 kg including thermal blankets). The overall external dimensions, with the door closed, were approximately $840 \times 550 \times 260 \mathrm{~mm}$.

\subsection{The Forward and Perimeter Baffles Assembly}

The forward and perimeter baffles support panel is a single CFRP composite panel with a large central cutout (see Figure 21), mounted to the base of the main structure by four aluminium mounting legs, again designed to provide an approximation to a semikinematic mount.

The baffle vanes were manufactured by bonding together a number of CFRP sheets, each having a quasi-isotropic layup, to build up a total thickness of about $1 \mathrm{~mm}$. The resultant solid CFRP material, which also has a very low coefficient of thermal expansion, was then cut to the required shapes and sizes.

It is worth noting that the diffractive rejection of stray light by the forward and perimeter baffles does not require the upper edge to be a perfect knife-edge - indeed sharp knife-edges would be problematic in terms of $i$ ) vulnerability to handling damage and ii) difficulty of ensuring adhesion of the paint film at the edges when the baffles are painted. A blunt knifeedge profile on each vane was produced by first machining the top edge at an angle of $45^{\circ}$ 
Figure 21 The forward and perimeter baffles support panel with the baffles installed.

Figure 22 The profile of the forward and perimeter baffle vanes.
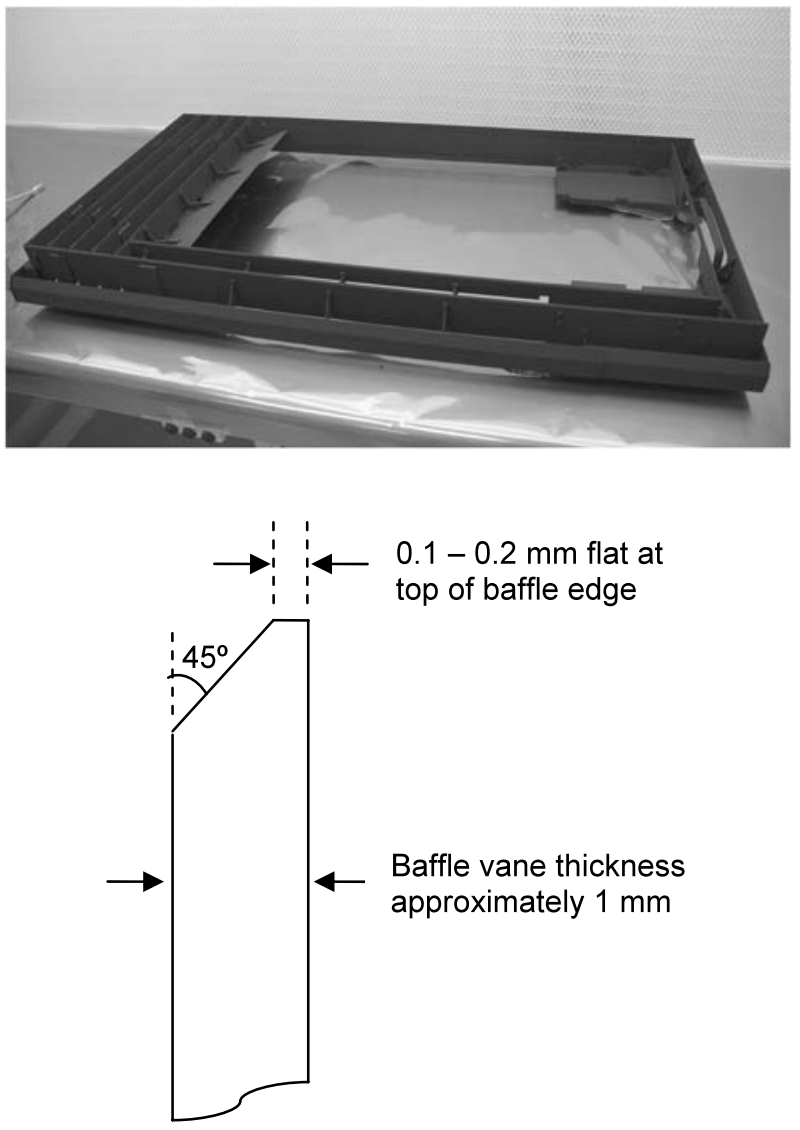

to the plane of the baffle vane, and then machining from above to give a $0.1-0.2 \mathrm{~mm}$ wide flat (see Figure 22).

The dimensions of the forward baffles are particularly critical - the height of each vane must be controlled to better than $65 \mu \mathrm{m}$ relative to its neighbours to maintain the required geometry for stray-light rejection by the five cascaded baffles. A maximum error budget of $20 \mu \mathrm{m}$ was allocated for manufacturing, to leave a good margin for long-term dimensional changes and thermally induced distortions in orbit (see Section 6.6). To achieve this accuracy, the final machining of the baffle edges was done with the baffle assembly mounted in the structure, by using an iterative precision machining procedure that involved the use of a computer numerical controlled milling machine, together with a coordinate measuring machine $(\mathrm{CMM})$ accurate to $2-3 \mu \mathrm{m}$.

\subsection{The Internal Baffle Assembly}

The dimensional tolerance requirements for the internal baffle assembly are much less stringent (typically $0.5-1 \mathrm{~mm}$ ) although a complex geometry is required, so a different manufacturing technique was used. The individual component parts of the baffle were formed from CFRP by laying up woven preimpregnated carbon fabric (T300) over aluminium moulds and then curing in the autoclave. These parts were then assembled by screwing and bonding them 
Figure 23 The internal baffle assembly before black painting of the outer surfaces.

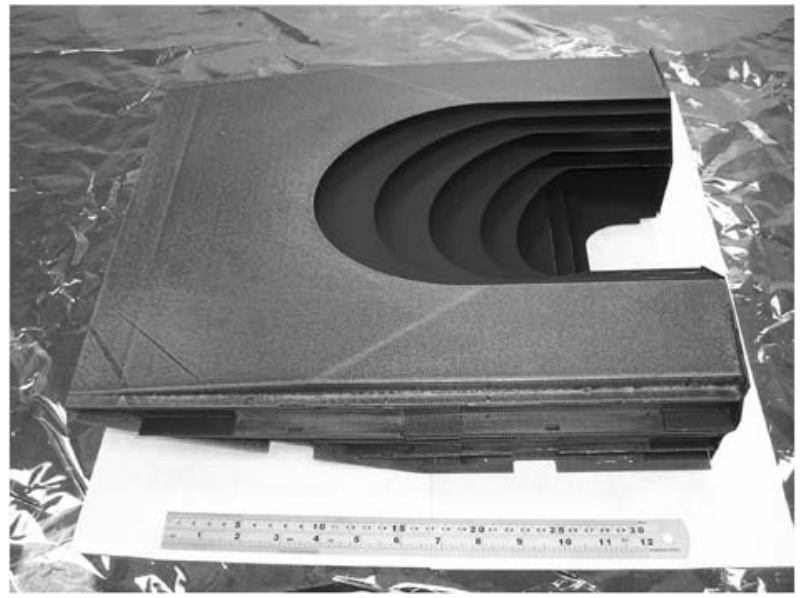

together to form a single, strong, and lightweight "monocoque" structure (see Figure 23). The thickness of the baffle vanes was typically $0.2-0.3 \mathrm{~mm}$ and there was no requirement to profile their edges.

The internal baffle is mounted directly to the sides of the main structure with small brackets. A system of shims (spacers whose thicknesses were iteratively adjusted by machining) and dowel pins passing through match-drilled holes was used to set up and maintain the alignment of the assembly relative to the structure.

\subsection{The Focal Plane Assembly Mounting Interfaces}

The two FPAs are mounted onto titanium right-angled strips attached to the load-bearing longitudinal bulkheads (see Figure 19). Small rectangular blocks are clamped to the strips, and these blocks in turn clamp cylindrical spigots that interface with the FPA housings (see Figures 13 and 14). On one bulkhead the spigots are free to move axially within the blocks, whilst on the other bulkhead they are constrained in all degrees of freedom - this is so that differential thermal expansion between the FPA housing and the surrounding structure does not cause excessive stresses to be imposed on the structure.

Again, a system of shims and dowel pins passing through match-drilled holes was used to set up and maintain the alignment. This arrangement permitted the telescope pointing direction to be aligned in all three axes of rotation, in addition to setting the location of the entrance aperture of the optics relative to the various baffle subsystems.

\subsection{The Door and Latch Mechanism}

The instrument was protected against ingress of contamination and handling damage during ground operations, launch, and the initial commissioning phase of the mission by a door (see Figure 3).

The door was fabricated from a CFRP panel with solid CFRP side pieces and is attached to the main structure by means of hinges that have redundant bearing surfaces. The door was held in the closed position by means of a latch mechanism that uses a StarSys Inc EP-5025 paraffin wax actuator. In orbit, the door operated as a one-shot system being released once only after the initial outgassing phase of the mission and with no capability to relatch. 


\subsection{Mechanical Design Analysis and Verification}

A finite-element model was used to verify the mechanical design of the instrument against the spacecraft requirements, namely,

- lowest resonant frequency above $50 \mathrm{~Hz}$ and

- quasistatic loads of $25 \mathrm{~g}$ applied separately in three orthogonal axes, with resultant factors of safety of

$\circ \geq 2.0$ for composite materials and

$\circ \geq 1.3$ yield (and $\geq 1.4$ ultimate) for other materials.

In addition to the use of the model to verify the design against these mechanical requirements (i.e., modal, static, and dynamic analyses), it was also used to predict the magnitude of thermal distortions caused by the large temperature changes between ambient and in-orbit operating conditions and the large temperature gradients across the instrument structure inorbit, by using predictions from the thermal modelling (see Section 7).

As a result of these studies, a number of modifications were made to the detailed design of the forward baffle assembly to ensure that changes of the relative baffle heights induced by thermal distortion under worst-case operating conditions are less than $20 \mu \mathrm{m}$. As discussed previously, $20 \mu \mathrm{m}$ of the overall $65 \mu \mathrm{m}$ budget for the relative heights of the forward baffle edges was allocated to manufacturing tolerances, so that $25 \mu \mathrm{m}$ remains for any long-term changes in the dimensions of the baffles.

\subsection{Instrument Alignment and Metrology}

During the assembly of the flight instruments, extensive metrology was performed to set up and align the various critical subsystems and components. The CMM used was the Mitutoyu model CRT-A910, which provides a measurement accuracy of $2-3 \mu \mathrm{m}$ over relatively short distances, degrading to $4-5 \mu \mathrm{m}$ over distances in excess of $0.5 \mathrm{~m}$.

To perform these measurements, the instrument was mounted on a substantial aluminium tooling plate (approximately $1 \mathrm{~m} \times 0.6 \mathrm{~m} \times 50 \mathrm{~mm}$ thick), which simulated the spacecraft mechanical interface, in addition to providing a reference datum for the measurements. Procedures were developed for measuring the following:

- directions of the HI-1 and HI-2 optical axes (and rotations about these axes) relative to the simulated spacecraft coordinate system,

- relative locations of the critical subsystems (forward baffles, side and rear baffles, internal baffles, and FPA housings), and

- relative locations of the various baffle edges within each baffle subsystem.

As discussed previously, the most critical dimensional requirements are the relative heights of the forward baffle vanes. The relative heights of the perimeter baffles are specified to $0.1 \mathrm{~mm}$, whilst the positions of the internal baffle vanes must be controlled to typically $0.5-1 \mathrm{~mm}$. Similar requirements apply to the relative positioning of the different baffle subassemblies and the optics entrance apertures.

It should be noted that setting up the alignments of the optical axes was performed by mechanical metrology using various reference points on the FPA housings and the lens barrels. Given the precision to which the various components of these subsystems were manufactured, this provided sufficient accuracy to ensure that overall instrument requirements are satisfied. The precise alignments were confirmed by optical measurements during instrument calibrations at CSL and subsequently refined in orbit by using background stars in the field of view (Brown, Bewsher, and Eyles, 2009). 
Table 4 Summary of temperature requirements $\left({ }^{\circ} \mathrm{C}\right)$.

\begin{tabular}{|c|c|c|c|c|c|c|}
\hline \multirow[t]{2}{*}{ Subsystem } & \multicolumn{2}{|c|}{$\begin{array}{l}\text { Operational } \\
\text { door closed }\end{array}$} & \multicolumn{2}{|c|}{$\begin{array}{l}\text { Operational } \\
\text { door open }\end{array}$} & \multicolumn{2}{|c|}{ Survival } \\
\hline & Min. & Max. & Min. & Max. & Min. & Max. \\
\hline Structure & -120 & 100 & -120 & 100 & -120 & 100 \\
\hline CCDs & -120 & 100 & -100 & -70 & -120 & 100 \\
\hline Lens barrels & -20 & 60 & -40 & 30 & -52 & 60 \\
\hline CEB & -35 & 50 & -35 & 50 & -50 & 60 \\
\hline Wax actuator & -50 & 40 & -120 & 80 & -120 & 80 \\
\hline
\end{tabular}

During all postdelivery ground operations (i.e., calibrations and instrument-level qualification testing at CSL, integration onto the spacecraft, spacecraft-level qualification testing, and launch preparations) a subset of the metrology was repeated at key intervals using a FARO portable CMM. Although this did not have the same accuracy as the machine used during instrument assembly, the $25-\mu \mathrm{m}$ accuracy of the portable CMM still provided a valuable confirmation that no major changes had occurred.

\section{Thermal Design}

The operational orbits for the two spacecraft are heliocentric, with STEREO-A being somewhat inside the Earth's orbit (at $0.95-0.97 \mathrm{AU}$ ) and STEREO-B somewhat outside (at 1.00$1.09 \mathrm{AU}$ ). The HI instruments are Sun-pointed during normal operations with the solar load, ranging from 1152 to $1654 \mathrm{~W} \mathrm{~m}^{-2}$, incident directly on their $+X$ (Sun-facing) faces. In operational mode there is electrical dissipation of approximately $10 \mathrm{~W}$, primarily in the CEB. The instrument temperature requirements are summarised in Table 4.

A thermal design using multilayer insulation, heaters, and passive radiators was developed. This design and the related computer models were validated by using data from thermal testing of the instrument. The instrument is thermally isolated from the spacecraft by the low thermal conductance titanium (Ti6Al4V) mounts. With the exception of the two radiators on the antisunward side of the instrument, the outer surface is insulated by thermal blankets having a black kapton outer layer. Once the door has opened, the black-coated baffles and cameras are exposed to deep space. The black-painted radiators are used for cooling the CCDs. As described in Section 4.2, each radiator is connected to the CCD by a cold finger, which is also connected to a cold cup. The cold cup is designed to remain colder than the $\mathrm{CCD}$, which it partly surrounds, protecting it from contamination and radiated heat loads.

Survival heaters, designed to provide thermal protection in nonoperational modes, are fitted to the CEB and the camera lens barrels. The heater at the CEB is thermostatically controlled, whilst those at the cameras are a fixed power that is dependent on the bus voltage. The lens barrel survival heater circuit incorporates a thermostat that closes as a result of lower structure temperatures when the instrument door has opened. This thermostatic switch enables a greater heater power when the door is open (and heat losses are greater) and a lower heater power when the door is closed (thus preventing overheating). In addition, operational heaters are fitted to the lens barrels to control their temperatures in operational modes. The survival heaters may be used in operational mode and this provides further margin on heater power at the lens barrels. Decontamination or bakeout heaters are fitted near the CCDs. As described in Section 5.2, a number of temperature sensors are fitted to the instrument, 
Table 5 Summary of cold and hot operational thermal analysis cases (door open).

${ }^{\mathrm{a}}$ Cold bounding case is for $\mathrm{HI}$ instrument on STEREO-B and hot bounding case is for instrument on STEREO-A.

${ }^{\mathrm{b}} \mathrm{BOL}$ and EOL are

beginning-of-life and end-of-life properties, respectively.

Table 6 Summary of thermal model predictions (door open).

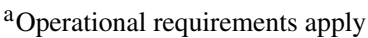
to all items shown, with the exception of the wax actuator.

${ }^{\mathrm{b}}$ Heaters are used to control lens barrel temperatures.

\begin{tabular}{|c|c|c|}
\hline Description of thermal case & Cold & Hot \\
\hline Applicable instrument ${ }^{\mathrm{a}}$ & HI-B & HI-A \\
\hline Bus voltage $[\mathrm{V}]$ & 26.5 & 35 \\
\hline Spacecraft interface temperature $\left[{ }^{\circ} \mathrm{C}\right]$ & -13 & 45 \\
\hline Sun distance $[\mathrm{AU}]$ & 1.089 & 0.909 \\
\hline Solar flux $\left[\mathrm{W} \mathrm{m}^{-2}\right]$ & 1152 & 1654 \\
\hline Radiative properties ${ }^{b}$ & BOL & EOL \\
\hline
\end{tabular}

\begin{tabular}{lll}
\hline Subsystem $^{\mathrm{a}}$ & Cold case $\left[{ }^{\circ} \mathrm{C}\right]$ & Hot case $\left[{ }^{\circ} \mathrm{C}\right]$ \\
\hline Structure & -69 to -53 & -56 to -33 \\
HI-1 CCD & -84 & -81 \\
HI-2 CCD & -83 & -80 \\
HI-1 lens barrel $^{\mathrm{b}}$ & -28 & -28 \\
HI-2 lens barrel $^{\mathrm{b}}$ & -28 & -28 \\
CEB internal $_{\text {Wax actuator }}$ & -18 & -6 \\
\hline
\end{tabular}

including thermistors on the lens barrels to provide the feedback mechanism for controlling the heaters.

Thermal design cases were derived by taking the extremes of the cold and hot parameters that the instruments may experience during the mission. The cold and hot thermal design cases for operational modes are summarised in Table 5.

Table 6 summarises the predictions for these two cases. It can be seen that the HI instrument has a rather unconventional thermal design and implementation in that the majority of the instrument runs very cold, with local heaters being used to raise the temperatures of individual subsystems to their operational requirements. To use heaters to raise the temperature of the entire instrument to more "normal" operating values would have required an unreasonable amount of heater power.

It can be seen that all predicted temperatures had adequate margin with respect to the applicable requirements. The lens barrel heaters are used to warm the optics to a temperature above the minimum requirements. It is noted that both survival and operational heaters were predicted to be required under certain conditions, but a margin was shown between predicted and available heater power in all required thermal cases.

\section{Instrument Development, Calibration, and Qualification Testing}

\subsection{Model Philosophy}

The HI instruments were developed following essentially a proto-flight model approach. However, to reduce risk, prototypes or engineering qualification models (EQMs) of critical subsystems were manufactured and subjected to appropriate testing at the subsystem level:

- A prototype forward baffle assembly was built and subjected to stray-light tests to verify the diffractive rejection performance of the concept (see Section 8.2). 
Figure 24 The prototype forward baffle assembly.

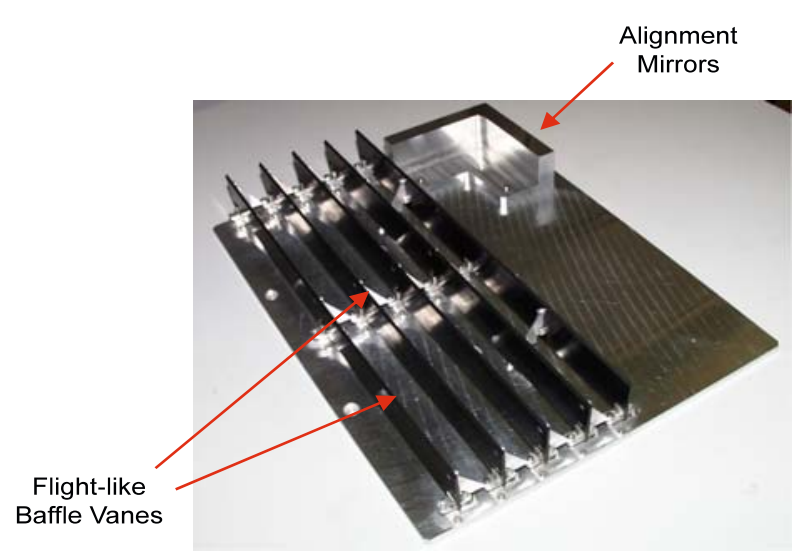

- An EQM of the main structure, including the mounting legs, door, hinges, and latch mechanism, was manufactured. This was partly to develop fabrication and assembly techniques for the CFRP structure. The structure was then fitted with EQMs of the lens assemblies, the CEB, and the HI-2 FPA mechanical components, together with mass dummies to simulate the mechanical properties of other subsystems. This assembly was subjected to mechanical qualification testing (vibration and shock tests). Door deployment tests under thermal vacuum were also performed.

- Lens assembly EQMs were subjected to thermal vacuum qualification at the unit level, in addition to the mechanical qualification when mounted in the EQM structure. The optical performance was verified at CSL before and after the testing.

- An EQM comprising the HI-2 FPA mechanical components was subjected to thermal vacuum qualification at the unit level, in addition to the mechanical qualification. A thermal balance test was then performed, primarily to verify the integrity of the two-part cold finger, with its clamped joint.

The development of the CEB followed a conventional full model philosophy, with prototyping, development model, EQM, and flight models (FMs), and with appropriate functional and qualification testing performed on the different models.

\subsection{Measurement of Stray-Light Rejection of the Prototype Forward Baffle}

The rejection of direct solar light by the forward baffle is key to the overall stray-light performance of the instrument, so at an early stage in the hardware development a prototype forward baffle assembly was built and tested to confirm the theoretical predictions for the diffractive rejection.

Figure 24 shows the prototype forward baffle. The baffle vanes were of the same dimensions (vane heights and separations) and were manufactured in exactly the same way as the flight baffles (see Section 6.2 and Figure 22). However, they were supported on an aluminium base plate, which was also provided with reference mirrors for setting up and alignment. The entire assembly (excluding the mirror faces) was painted with the same matte black paint as used on the flight models.

The test configuration used for the measurements at CSL is shown in Figure 25. The baffle prototype was mounted within a black enclosure, which in turn was within a vacuum chamber. An intense light source was provided by a laser diode with an optical fibre feed. The detector, which could be remotely rotated through a range of angles relative to the 


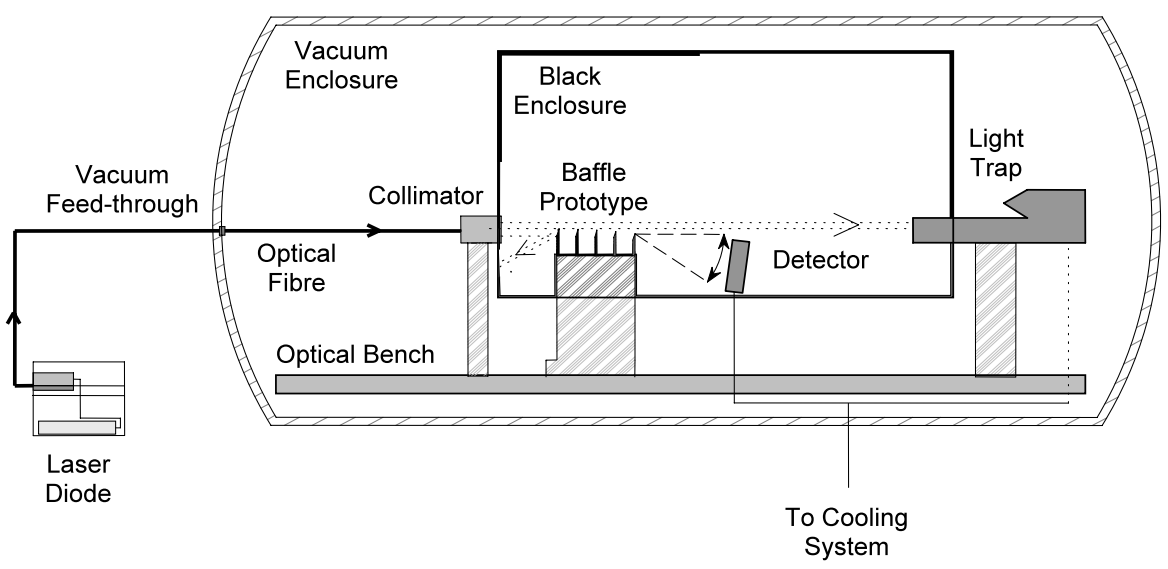

Figure 25 The test configuration for the prototype forward baffle diffracted light measurements.

incoming beam, was a Hamamatsu photomultiplier tube, cooled to $-40^{\circ} \mathrm{C}$. To measure the very low levels of diffracted light, rigorous precautions were taken to prevent light scattered within the chamber from reaching the detector - a light trap was used to absorb the direct beam from the source that passed over the top of the baffles, and particular attention was paid to the blackening of those walls of the enclosure that were in the field of view of the detector and to suppress light scattered off the front face of the first baffle vane.

The results obtained are shown in Figure 26. Measurements were made initially at ambient pressure and then under vacuum, to eliminate the possibility of scattering by airborne dust particles. The rejection over the angular offset range corresponding to the HI- 1 entrance aperture is $10^{-9}$ to $10^{-11} B / B_{\odot}$, whilst for HI-2 it is $\approx 3 \times 10^{-12}$. The results generally lie fairly close to the theoretical curve, and the improvement resulting from making the measurements under vacuum is clearly evident at rejection levels below $10^{-11}$. The agreement with the theoretical curve at angles larger than $4^{\circ}$ is impressive, and the ability to make stray-light rejection measurements down to these levels is a remarkable achievement.

\subsection{Performance Testing of the FM Lens Assemblies}

Prior to their installation into the telescope assemblies, the FM lenses were subjected to a number of acceptance and performance tests at CSL. These included the following:

- measurement of the wave-front error using an interferometer,

- determination of the focal plane position with respect to the lens barrel mounting interfaces, on the basis of minimising the wave-front error (which, together with metrology on the FPAs, enabled the spacers that set the focus of the telescopes to be adjusted),

- measurement of the absolute transmission as a function of wavelength, and

- measurement of the stray-light rejection of out-of-field objects by the lens barrels.

Results for these last two items are presented in the subsequent sections.

\subsubsection{Measurement of Absolute Transmission of the FM Lens Assemblies}

Figure 27 shows the measured absolute transmission of the four FM lens assemblies. The agreement between the HI-1 measured curves and the manufacturer's predicted curve from 


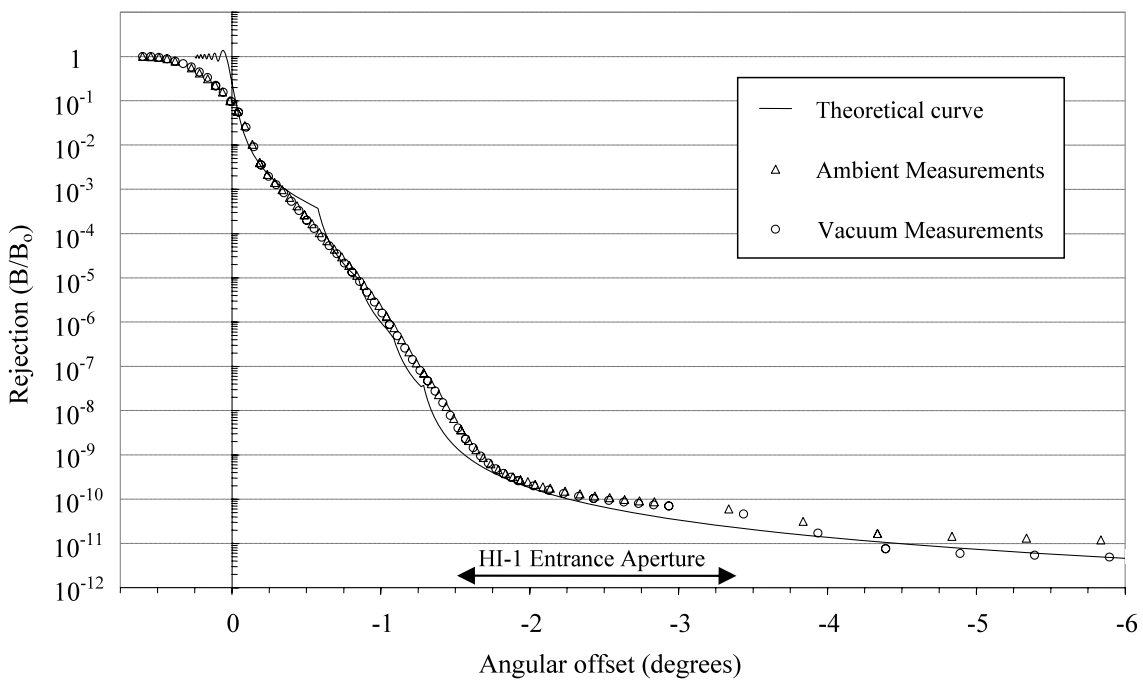

Figure 26 The measured stray-light rejection of the prototype forward baffle assembly as a function of offset angle relative to the incoming beam, together with the theoretical prediction.

the filter coatings design is very good. The agreement for HI-2 is not so good, with the measurements systematically lower than predicted and the peak transmission at a somewhat higher wavelength. The reason for these discrepancies is not known; however, it is not regarded as serious in that the overall photometric response of the HI telescopes is being calibrated in orbit by using the response to "standard" stars.

\subsubsection{Measurement of Stray-Light Rejection by the FM Lens Assemblies}

A measurement was made of the out-of-field stray-light rejection of the lens assemblies prior to integrating them with the FPAs in the telescope assemblies. The measurement was performed by mounting a $1 \times 1 \mathrm{~cm}$ square photodiode detector (Newport 818-SL) at the focal plane and moving a light source over a range of angles both within and outside the field of view. Since this detector is considerably smaller than the HI CCD detector, the effective field of view is also much smaller. This was useful in that it enabled the stray-light contribution by scattering within the optics to be quantified for the outer regions of the HI camera fields of view, as well as outside the fields.

The results are shown in Figure 28. It can be seen that at the angles corresponding to the edges of the field of view $\left(10^{\circ}\right.$ and $\left.35^{\circ}\right)$ the stray-light contribution levels are $(1-2) \times 10^{-3}$ and $<10^{-4}$ for HI- 1 and HI-2, respectively. These values, together with the measured rejection of the prototype forward baffles (see Section 8.2 and Figure 26), provided an extremely important verification that the overall stray-light rejection requirements of the instrument, given in Table 1, would be satisfied with substantial margins.

\subsection{Instrument Qualification Testing and Optical Calibrations}

The optical calibrations and the majority of the qualification testing of the FM instruments were carried out at CSL. The test campaigns included the following stages:

- initial alignment and metrology checks, 
Figure 27 The measured absolute transmission efficiencies as a function of wavelength for

(a) HI-1 lens assemblies and

(b) HI-2 lens assemblies, compared with the manufacturer's predicted curves.
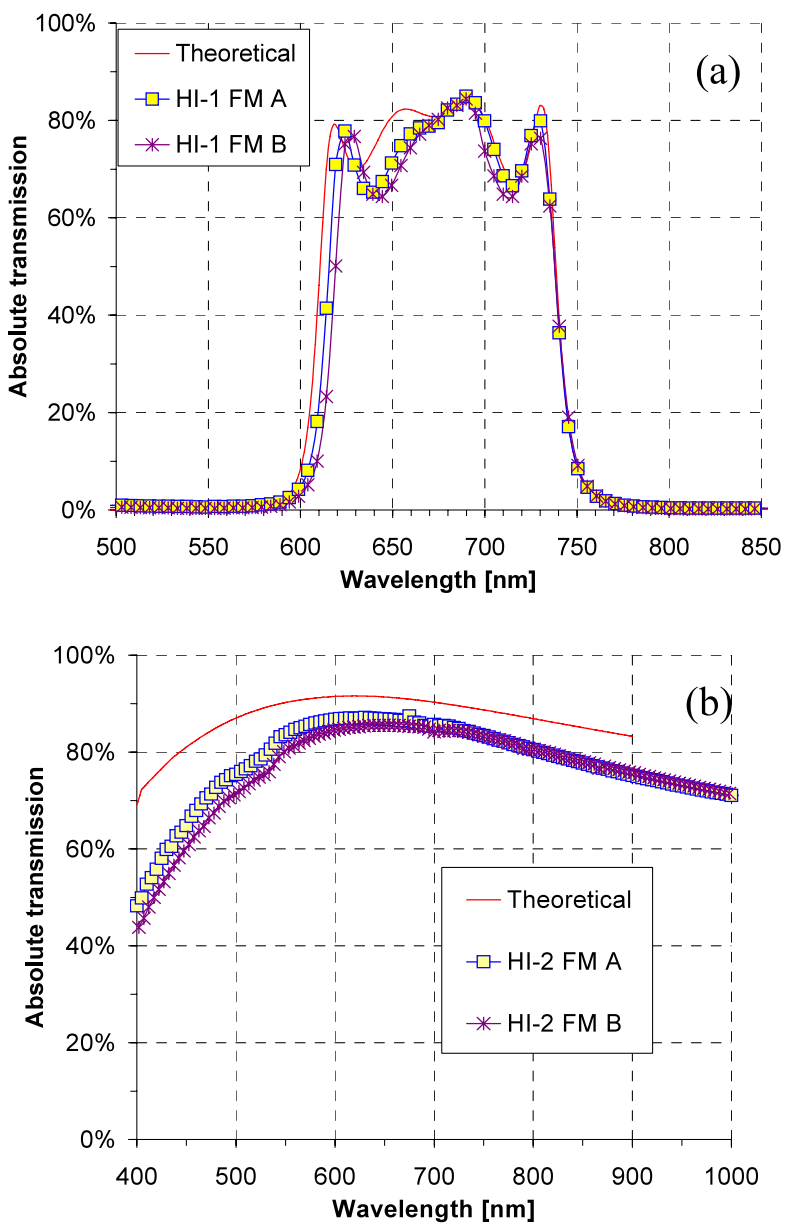

- vibration test (at proto-flight levels of $10.4 g_{\text {rms }}$ perpendicular to instrument mounting plane and $7.4 g_{\text {rms }}$ in plane of instrument),

- repeat of alignment and metrology checks,

- door deployment test in flight configuration with thermal blankets fitted and under thermal vacuum conditions to simulate in-orbit deployment,

- thermal balance test (HI-B only),

- thermal vacuum testing including hot and cold survival soaks and a number of operational cycles,

- optical calibration,

- solar stray-light rejection measurements (HI-B only),

- final thermal vacuum bakeout, and

- repeat of alignment and metrology checks.

The thermal qualification tests and the optical calibrations were carried out in the same vacuum chamber. During all these tests the CCDs were conductively cooled to around $-110^{\circ} \mathrm{C}$ by connecting thermal links from a liquid nitrogen dewar to the cold fingers, so that the cameras could be read out with a very low noise level. During the thermal qualification 
Figure 28 The measured out-of-field stray-light rejection for (a) HI-1 lens assemblies and (b) HI-2 lens assemblies, using a $1 \times 1 \mathrm{~cm}$ square detector, rather than the $27.6 \times 27.6 \mathrm{~mm} \mathrm{HI}$ CCD.
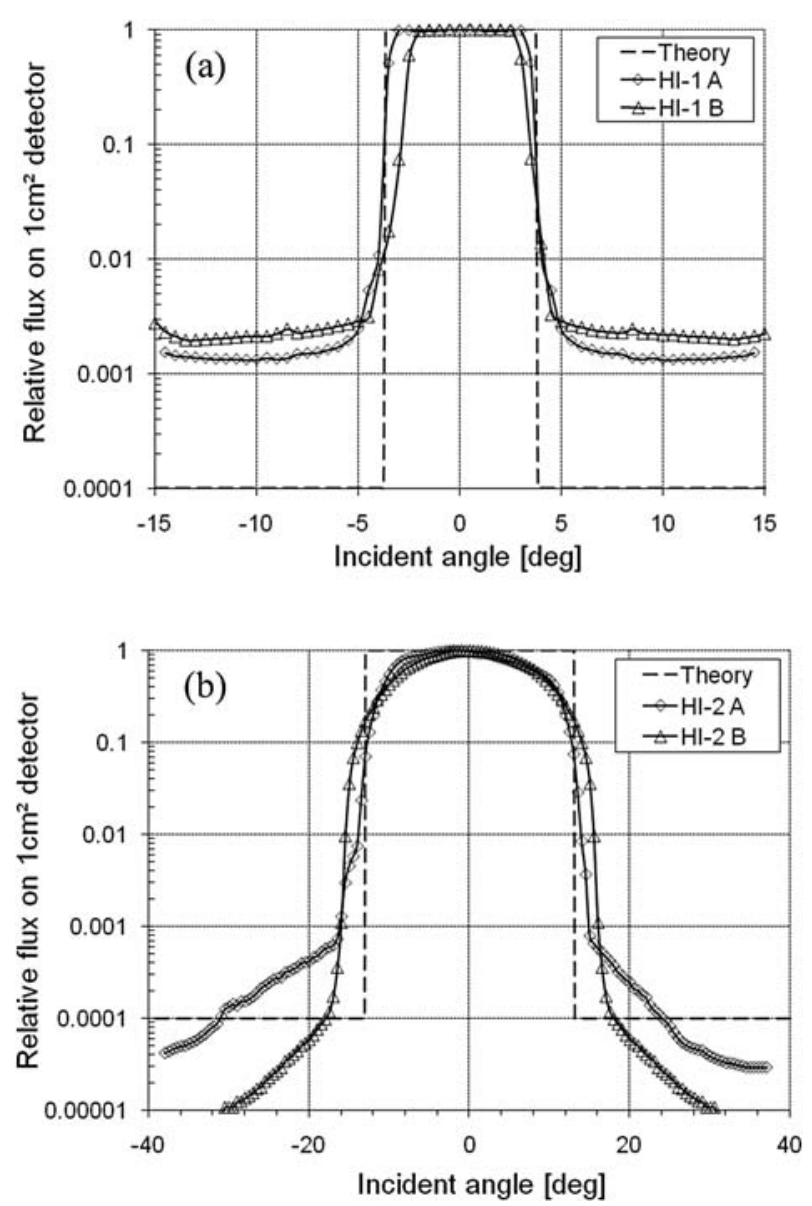

tests, automatic image readout was performed at a cadence similar to that used in flight operations, in addition to functional testing at appropriate intervals.

Figure 29 shows HI-A installed in the vacuum chamber. The instrument was mounted on a remotely controlled rotary platform so that it could be rotated about a vertical axis. This allowed a horizontal light beam from a collimator to be scanned across the centreline of the HI-1 and HI-2 fields of view. The collimator, which was located in a separate cylindrical chamber connected to the main chamber via a port, was $300 \mathrm{~mm}$ in diameter and $F / 10$, providing a collimated beam with 30 arc-sec divergence when fed from a light source via a $400-\mu \mathrm{m}$ core optical fibre placed at the collimator focus. The light source was either an Ocean Optics HL-2000-HP halogen source or a 2-W output (continuous) laser diode with a wavelength of $670 \mathrm{~nm}$, depending on the test being performed.

The optical calibrations performed can be summarised as follows:

- Alignment measurements:

- Measurement of HI-1 and HI-2 boresight directions relative to the instrument mounting interface, and measurement of the relative offset between the HI-1 and HI-2 boresights.

- Scans of the collimated light source (i.e., the image spot) along the centreline of the HI-1 and HI-2 fields of view: 


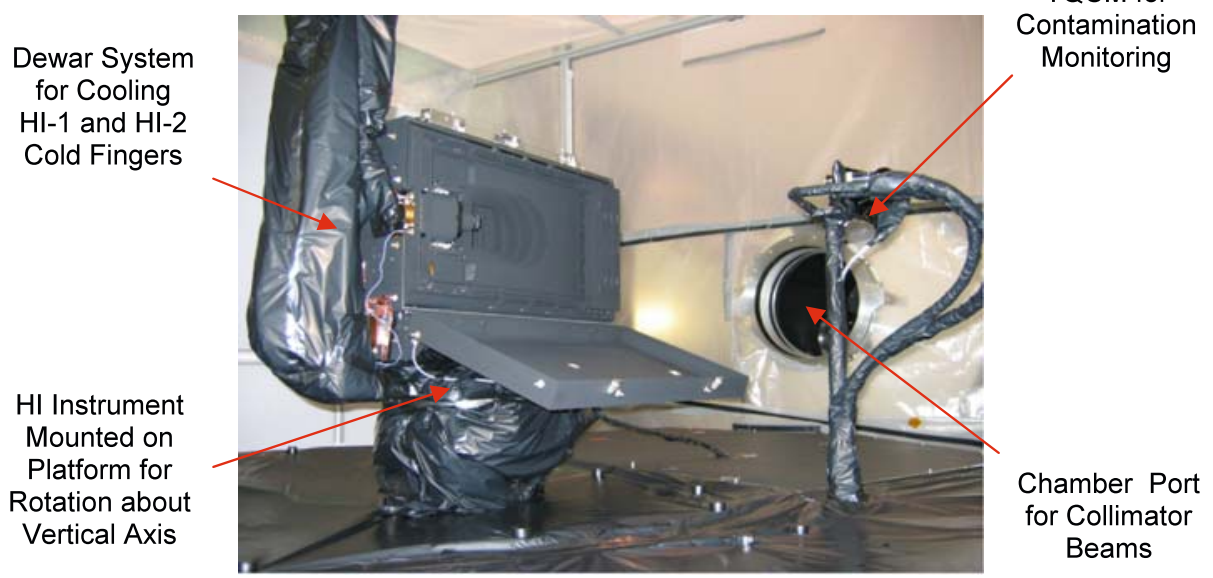

Figure 29 The HI-A instrument installed in a vacuum chamber at CSL for thermal vacuum qualification testing and optical calibration.

- Measurement of the centroid position of the image of the calibration source on the CCD versus the angle of the rotary platform, enabling the plate scales, focal lengths. and distortion parameters of the cameras to be determined.

- Characterisation of the point-source response function (PSF) of the cameras, including measurement of the half-energy width, as a function of position in the field of view in each camera.

- Measurement of the flux within the image spot as a function of the angle of the rotary platform, enabling the variation of response across the field of view (the large-scale flat field) to be determined.

- Characterisation of the ghosts associated with bright objects both within and outside the field of view.

- An end-to-end test of the rejection of solar stray light (HI-B only).

Table 7 summarises the main results of the optical calibrations. The results for the focal length and distortion parameters, the large-scale flat field, and the stray-light rejection measurements are discussed in more detail in the subsequent sections.

\subsubsection{Measurements of Focal Length and Distortion Parameters}

Traditionally, almost all solar imaging instruments, including coronagraphs, have used the gnomonic or tan projection. In this projection, an object at an angle $\alpha$ to the instrument optical axis, or boresight, is projected onto the image plane at a radial distance $R$ from the boresight position given by

$$
R=F \tan (\alpha),
$$

where $F$ is the focal length of the optical system and angular symmetry about the optical axis is assumed. The position of an object in the image plane is then given in terms of an appropriate rotation angle $\beta$ by

$$
\begin{aligned}
& x=F \tan (\alpha) \cos (\beta), \\
& y=F \tan (\alpha) \sin (\beta) .
\end{aligned}
$$


Table 7 HI-1 and HI-2 optical systems main characteristics.

\begin{tabular}{|c|c|c|c|}
\hline & Design values & FM-A & FM-B \\
\hline \multicolumn{4}{|l|}{ HI-1 } \\
\hline Optical axis - pitch $^{\mathrm{a}}$ & $-13.65^{\circ}$ & $-13.64^{\circ}$ & $-13.67^{\circ}$ \\
\hline yaw & $0^{\circ}$ & $-0.15^{\circ}$ & $-0.49^{\circ}$ \\
\hline roll & $0^{\circ}$ & $0.90^{\circ}$ & $1.02^{\circ}$ \\
\hline Focal length (paraxial) & $78.46 \mathrm{~mm}$ & $77.54 \mathrm{~mm}$ & $77.65 \mathrm{~mm}$ \\
\hline Pixel size (paraxial) & 35.50 arc-sec & 35.92 arc-sec & 35.87 arc-sec \\
\hline Distortion parameter $(\mu)$ & - & 0.1668 & 0.1000 \\
\hline Distortion $^{b}$ & $<1 \%$ & $0.83 \%$ & $0.91 \%$ \\
\hline HEW - on axis & $45.2 \mu \mathrm{m}$ & $38.8 \mu \mathrm{m}$ & $37.9 \mu \mathrm{m}$ \\
\hline edge of field ${ }^{b}$ & $67.6 \mu \mathrm{m}$ & $54.3 \mu \mathrm{m}$ & $49.8 \mu \mathrm{m}$ \\
\hline Response at edge of field ${ }^{c}$ & $98.5 \%$ & $-d$ & $95.8 \%$ \\
\hline \multicolumn{4}{|l|}{ HI-2 } \\
\hline Optical axis - pitch $^{\mathrm{a}}$ & $-53.35^{\circ}$ & $-53.13^{\circ}$ & $-53.31^{\circ}$ \\
\hline yaw & $0^{\circ}$ & $-0.02^{\circ}$ & $-0.61^{\circ}$ \\
\hline roll & $0^{\circ}$ & $-0.02^{\circ}$ & $0.22^{\circ}$ \\
\hline Focal length (paraxial) & $21.67 \mathrm{~mm}$ & $21.46 \mathrm{~mm}$ & $21.52 \mathrm{~mm}$ \\
\hline Pixel size (paraxial) & 128.51 arc-sec & 129.77 arc-sec & 129.41 arc-sec \\
\hline Distortion parameter $(\mu)$ & - & 0.8333 & 0.6506 \\
\hline Distortion $^{b}$ & $<8 \%$ & $4.3 \%$ & $5.5 \%$ \\
\hline HEW - on axis & $105.3 \mu \mathrm{m}$ & $31.5 \mu \mathrm{m}$ & $68.6 \mu \mathrm{m}$ \\
\hline edge of field ${ }^{b}$ & $145 \mu \mathrm{m}$ & $78.1 \mu \mathrm{m}$ & $164.8 \mu \mathrm{m}$ \\
\hline Response at edge of field ${ }^{\mathrm{C}}$ & $81.9 \%$ & $-d$ & $82.0 \%$ \\
\hline
\end{tabular}

${ }^{\text {a }}$ Values for the pitch angle of the optical axis relative to the mounting interface of the instrument. To allow a margin for possible misalignments within the spacecraft structure, a further offset of 20 arc-min was introduced when mounting the instrument onto the spacecraft, resulting in overall offsets of $14.0^{\circ}$ and $53.7^{\circ}$ from Sun centre.

${ }^{b}$ Values at edge of the circular field of view.

${ }^{\mathrm{c}}$ Response at edge of the circular field of view relative to value on axis. The design values are based on the geometrical projected area of the entrance apertures only.

${ }^{\mathrm{d}}$ Owing to limitations of the test configuration, in particular the absence of a shutter at the light source, reliable results were not obtained for HI-A in these cases.

However, this representation is not adequate to describe the imaging properties of the HI cameras because of their wide-angle optics and the resultant distortion at the edge of the field of view, particularly in the case of HI-2.

Analysis of results from scans of the calibration source across the fields of view showed that the image projection of both cameras can be accurately represented by the relationship

$$
R=F_{\mathrm{p}} \frac{(\mu+1) \sin (\alpha)}{\mu+\cos (\alpha)}
$$

where $F_{\mathrm{p}}$ is the paraxial focal length and $\mu$ is a distortion parameter. The value of $F_{\mathrm{p}}$ may be used to calculate the paraxial plate scale $f_{\mathrm{p}}$.

Inspection of this relationship shows that, for $\mu=0$, it reverts to the simpler tan projection. Again, angular symmetry about the optical axis is implicit. It should be noted that 
Figure 30 Results for fitting the AZP distortion relationship to the data from the calibration scans for (a) HI-1B and (b) HI-2B. The available data points for HI-2B are limited by the presence of an Earth occulter in front of the $\mathrm{CCD}$ on the centreline of the field of view.
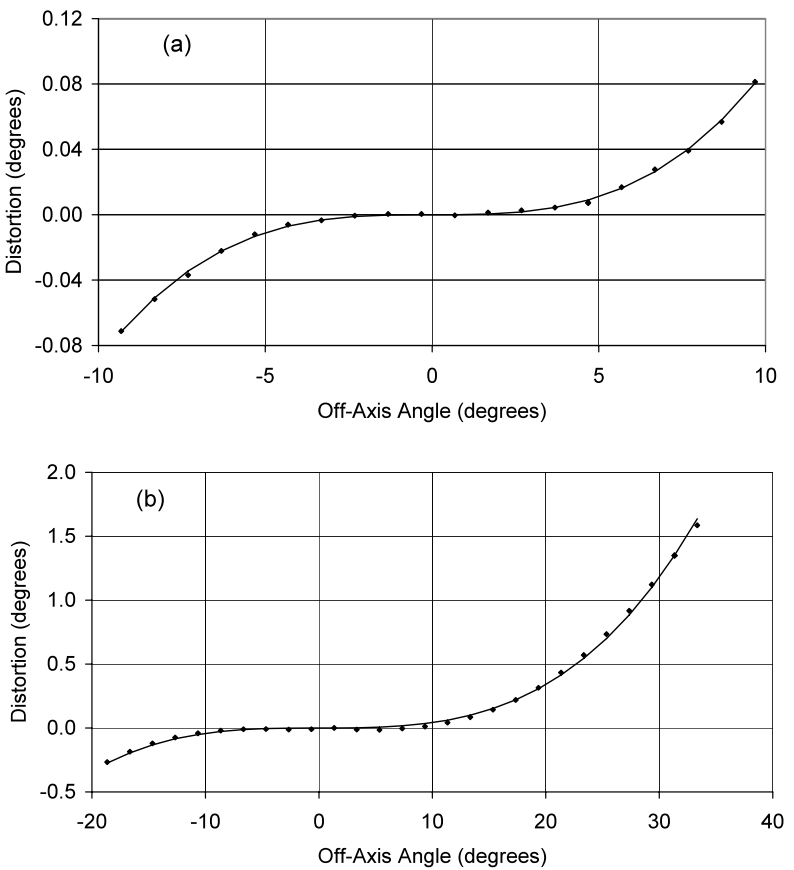

the relationship characterises the azimuthal perspective (AZP) projection as derived by Calabretta and Greisen (2002). However, there is no particular physical basis for applying this projection to the HI cameras; the relationship is used simply because it is a suitable twoparameter model that can accurately represent the behaviour of the optics, including the distortion at the edges of the field of view.

Table 7 gives the values for paraxial focal length (together with the corresponding paraxial plate scale in arc-sec per pixel) and distortion parameters obtained by fitting the AZP projection to the data from the calibration scans. Figure 30 shows the fits obtained for the HI-B cameras. In these plots the vertical axis represents the distortion expressed as the angular deviation from a linear plate scale.

\subsubsection{Measurements of Large-Scale Flat Fields}

The relative response across the camera fields of view was calibrated by measuring the total flux in the calibration image spot on the CCD as a function of angular position. The test configuration only permitted the calibration source to be scanned across the centreline of the field of view, so no information was obtained about the response in the corners of the fields.

The measured angular response was fitted to the function

$$
I=I_{0}[\gamma \cos (\alpha)+(1-\gamma)]
$$

where $I$ is the response at an angle $\alpha$ to the optical axis, and the best-fit values for the parameter $\gamma$ were 2.66 and 0.90 for $\mathrm{HI}-1 \mathrm{~B}$ and $\mathrm{HI}-2 \mathrm{~B}$, respectively. Hence, the variation of the response over the HI-2B (circular) field of view was very close to a simple geometrical projection of the entrance aperture area, although for HI-1B it decreased significantly faster. This is most likely due to the angular response of the various coatings on the optics. 
For the purposes of correcting images, it is more convenient to characterise the response as a function of distance $r$ from the centre of the CCD image. For both HI-1B and HI-2B, it was found that the response $I$ at a radial distance $r$ could be accurately represented by the polynomial

$$
I=I_{0}\left(1+a r^{2}+b r^{4}\right),
$$

where $I_{0}$ is the response on axis (as defined by the CCD centre).

With $r$ expressed in millimetres, the optimised values for $a$ and $b$ were $-2.18 \times 10^{-4}$ and 0.00 (actually $<10^{12}$ ) for HI-1B and $-6.24 \times 10^{-4}$ and $-1.65 \times 10^{-6}$ for HI-2B.

\subsubsection{Measurement of Stray-Light Rejection at the Instrument Level}

Although considerable confidence was gained from the tests at subsystem level that HI would meet the solar stray-light rejection requirements (see Sections 8.2 and 8.3.2), it was nevertheless considered important to perform an end-to-end verification of the completed instrument. Such a test was performed on HI-B as part of the optical verification and calibration.

The light source used for the stray-light tests on the instrument was the 2-W laser diode. In these tests an alternative $F / 3$ refractive collimator was used, providing an output beam of area $25 \times 5 \mathrm{~mm}$, with an intensity of $80 \mathrm{~mW} \mathrm{~cm}^{-2}$ and a divergence of 27.5 arc-min. The divergence closely matched that of the solar flux, whilst the intensity was of similar magnitude (total solar flux $\approx 150 \mathrm{~mW} \mathrm{~cm}^{-2}$ ). The beam was set up to be incident on the central region of the forward baffle - since the diffraction pattern is strongly forward-directed this was entirely adequate for the rejection measurements. Indeed, to have used a beam with larger dimensions would have compounded the challenges of trapping the unused flux and preventing it from contributing to the background light in the chamber.

To suppress the background stray light in the chamber sufficiently to reach the straylight rejection capabilities of the instrument, a number of enhancements were made to the test setup. For all the optical calibrations the instrument was enclosed within a five-sided structure, which was painted with matte black paint (Aeroglaze Z307). To reduce stray light further, a vacuum-compatible black velvet appliqué, VelBlack (manufactured by Energy Science Laboratories Inc., San Diego), was selectively applied to various internal surfaces of the five-sided enclosure. In particular, it was used to attenuate that part of the incident beam that passed over the top of the forward baffles and was also used to provide "super black" surfaces within the field of view of each camera. VelBlack has a bidirectional reflectance distribution function (BRDF) of $\leq 0.05 \%$ at $630 \mathrm{~nm}$, compared with $\approx 2 \%-3 \%$ for the black paint.

Figure 31 shows images obtained from the HI-1B and HI-2B cameras during the straylight tests. The direction of the laser light source is indicated, and in each case it is in the nominal Sun direction (i.e., $3.65^{\circ}$ and $18.35^{\circ}$ from the edge of the field of view). The images show the various features of the surroundings in the vacuum chamber as viewed by the cameras and illuminated by the ambient background light level from scattering of the incoming beam. For example, in the HI-1 image the front of the light source collimator is clearly visible, whereas in the HI-2 image the welded joints between the panels of the surrounding black-painted enclosure can be seen.

It should be noted that the light levels are extremely low, the images being deep exposures of 12-minute duration. The measured brightness as a function of the incident beam intensity at various points in the field of view is indicated in Figure 31. This was calculated 

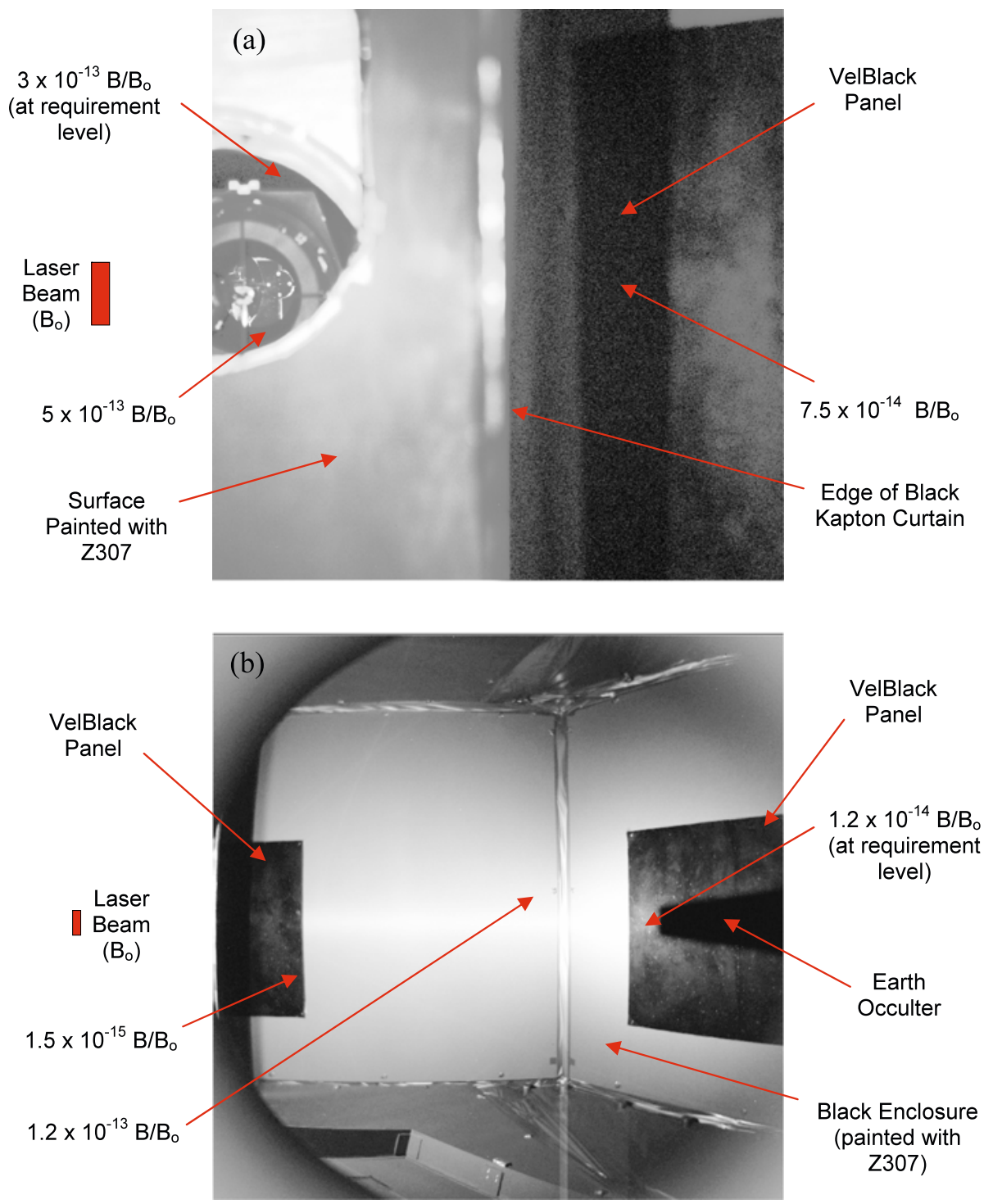

Figure 31 End-to-end stray-light evaluation images for (a) HI-1 and (b) HI-2 telescopes. The direction of the laser light source beam is shown, corresponding to the nominal Sun direction. The images show the various features of the surroundings in the vacuum chamber, illuminated by the background light. The rejection factor relative to the incoming beam at various points in the field of view is indicated, including regions where the stray-light rejection is at the requirements level.

from a knowledge of the incident beam intensity, together with estimates of the photometric responses of the cameras.

Generally, the detected levels were limited by the brightness of the various background features of the vacuum chamber and black enclosure in the fields of view of the cameras, and they represent upper limits on stray-light levels of the instrument itself. The contrast between the regions of the enclosure that were painted with the "black" paint and those 
where VelBlack was applied is striking. It can be seen that in regions where the background scattered light was suppressed sufficiently (e.g., by the VelBlack panels), the stray-light attenuation of the instrument is substantially in excess of the requirements.

In addition to this measurement of solar stray-light rejection, the response of the cameras to bright objects within the field of view was also assessed. The ghost images associated with such bright objects showed rejection factors better than $6 \times 10^{-9}$ and $3 \times 10^{-7}$ per pixel for HI-1 and HI-2, respectively.

\section{Launch, Instrument Commissioning, and Mission Operations}

The HI instrument checkout, commissioning, and early mission operations are described in detail in Eyles et al. (2007). The most important activities and results are described in the subsequent sections.

\subsection{Launch and Precommissioning Phase}

The twin STEREO spacecraft were launched aboard a single Delta II rocket from Cape Canaveral Air Force Base at 01:52 UT on 26 October 2007 (8:52 pm EDT on 25 October). Each spacecraft subsequently performed several highly eccentric Earth orbits, followed by a lunar swing-by to achieve heliocentric orbit insertion.

A few hours after launch, decontamination heaters were switched on to prevent volatile outgassing materials from condensing on the CCDs and optics surfaces. These heaters remained on for approximately one month following launch. During this period, functional checks were performed on the cameras, albeit with warm CCDs (around $30^{\circ} \mathrm{C}$ ).

After about one month, the decontamination heaters were switched off and the CCDs were cooled by the passive radiators over a period of $\sim 12$ hours, reaching final temperatures around $-60^{\circ} \mathrm{C}$. (The $\mathrm{HI}$ doors were still closed at this stage, so the temperatures reached were somewhat higher than the final operating values.)

During the CCD cool-down phase, images were taken at periodic intervals to characterise the CCD dark current as a function of temperature, thereby allowing the accumulated dark charge at the final CCD operating temperatures to be predicted. The results obtained are given in Section 9.1.1.

After switching off the decontamination heaters, it became possible to assess camera performance with cooled CCDs. During this period, priority was given to obtaining dark calibration images, together with images using the calibration LEDs, prior to opening the "one-shot" instrument doors.

\subsubsection{CCD Cooling Curves and Dark Charge Measurements}

Figure 32 shows the cooling curves for the HI-A CCDs when the decontamination heaters were turned off, together with the variation of dark current with temperature for HI-1A. The results for the other cameras were very similar.

The variation of dark current for a noninverted-mode CCD can be characterised by the relationship

$$
I_{\text {dark }}=A T^{-3} \exp (-6400 / T),
$$

where $T$ is the temperature in kelvins (http://www.e2v.com). 

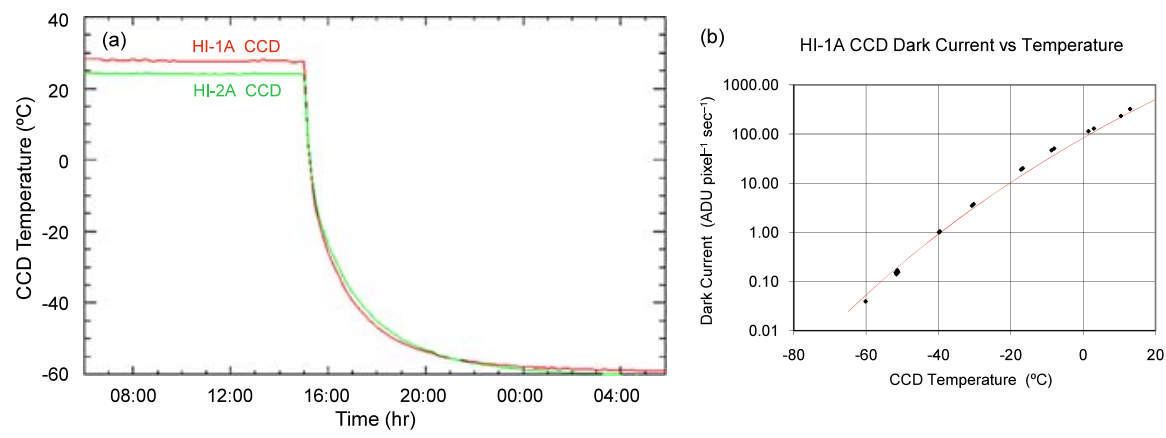

Figure 32 (a) Cooling curves for HI-1A and HI-2A CCDs. (b) HI-1A CCD dark current versus temperature.

Table 8 Predicted CCD dark current values at $-80^{\circ} \mathrm{C}$.

\begin{tabular}{llllll}
\hline CCD temperature & \multicolumn{4}{l}{ Dark current $($ ADU pixel } & \\
\cline { 2 - 5 } & HI-1A & HI-2A & HI-1B & HI-2B & e2v value $^{-1}$ \\
\hline$+20^{\circ} \mathrm{C}$ & 506 & 392 & 496 & 402 & 1300 \\
$-80^{\circ} \mathrm{C}$ & 0.0018 & 0.0014 & 0.0017 & 0.0014 & - \\
\hline
\end{tabular}

${ }^{\mathrm{a}}$ Calculated from e2v technologies value of $20000 \mathrm{e}^{-}$pixel $^{-1} \mathrm{~s}^{-1}$ using a CEB gain factor of 1 ADU $=$ $15 \mathrm{e}^{-}$

This relationship was fitted to the measured dark current values and the resultant best fit for HI-1A is shown in Figure 32(b). A good fit is achieved despite the wide range of temperatures. Similar fits were obtained for the other cameras. The fitted values for the normalisation constant $A$ were then used to predict the expected dark current at the final CCD operating temperatures of approximately $-80^{\circ} \mathrm{C}$.

The predicted values for dark current at $-80^{\circ} \mathrm{C}$ are given in Table 8 , together with measured values at $+20^{\circ} \mathrm{C}$. The nominal dark current at $+20^{\circ} \mathrm{C}$ as quoted by the CCD manufacturer ( $2 \mathrm{v}$ technologies) is also shown for comparison.

The predicted accumulated dark charge for a summed image at the final operating temperatures of approximately $-80^{\circ} \mathrm{C}$ can now be calculated, and it is found to be completely negligible. For the HI-2 summed image exposure times given in Table 10, the total charge is $\approx 30 \mathrm{DN}$ per image bin. Even in the darkest regions of a summed HI-2 image, the sky background signal is three orders of magnitude larger at $\approx 30000 \mathrm{DN}$ per bin. This is an excellent result, demonstrating that there is a large margin for possible degradation of CCD performance resulting from radiation damage. It is also noted that measured values of dark current at $+20^{\circ} \mathrm{C}$ are substantially lower than the manufacturer's quoted typical value.

\subsection{Instrument Commissioning Phase}

To avoid possible contamination of sensitive optical surfaces by condensable material from spacecraft thruster firings, the HI doors remained closed until after the final attitude manoeuvres prior to lunar swing-by and heliocentric orbit insertion for each spacecraft.

The HI-A door was opened on 13 December 2006, two days before STEREO-A lunar swing-by. The first-light images are shown in Figures 33(a) and (b). They are of outstand- 

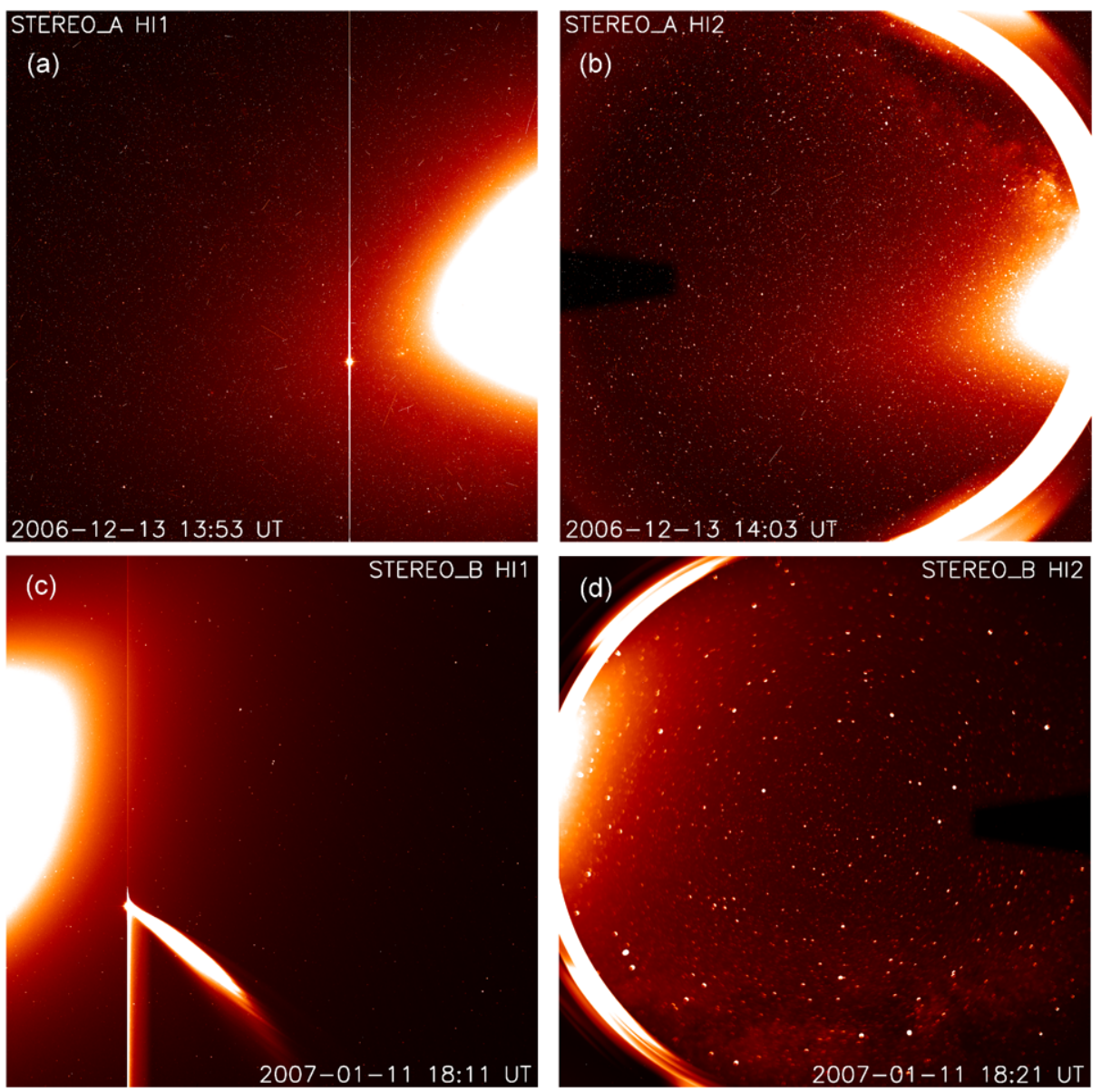

Figure 33 (a) HI-1A first-light image and (b) HI-2A first-light image from 13 December 2006. (c) HI-1B first-light image and (d) HI-2B first-light image from 11 January 2007.

ing quality, with the F-corona, Venus, the Milky Way, several clusters, and numerous stars visible.

The HI- $1 \mathrm{~A}$ image is $20^{\circ}$ across and the Sun is $4^{\circ}$ to the right of the frame. The F-coronal intensity is only $10^{-10} B_{\odot}$ at $4^{\circ}$, which in itself is a remarkable demonstration of the performance of the instrument. Venus in HI-1A is saturated, causing the CCD full well capacity to be exceeded and excess charge to bleed up and down the columns, as seen in the image.

The HI-2 image is $70^{\circ}$ across with the Sun $18.7^{\circ}$ off the right-hand edge. Here we are seeing the F-coronal intensity at levels of only $10^{-12} B_{\odot}$, although the coronal profile can be followed right across the field of view. The bright semicircular rings on the right-hand side are images of the internal baffle edges, illuminated primarily by stray light from the Earth.

The HI-B door was deployed on 11 January 2007, and the first-light images showed spectacular views of Comet McNaught (Fulle et al., 2007). The final lunar swing-by and heliocentric insertion of STEREO-B was on 21 January 2007.

Figures 33(c) and (d) show first-light images from HI-B. In this case the Sun is off the left-hand edge of the frames. At this early stage in the mission the spacecraft attitude was 
not aligned with the ecliptic, resulting in the appearance of the F-corona at an angle in the images. Although the coma of Comet McNaught in HI-1B is saturated, fine structure in the tail is clearly visible.

Following deployment of the doors, the temperature of the entire instruments dropped over a time scale of $\sim 12$ hours. CCD temperatures around $-80^{\circ} \mathrm{C}$ were achieved, giving very low dark charge and high tolerance to radiation damage effects, as discussed in Section 9.1.1. The in-orbit thermal performance of the instruments is described in more detail in Section 9.2.1.

After door opening, the mission entered the instrument commissioning phase. The synoptic observing sequences were tested and optimised, resulting in the final values given in Table 10 (see Section 9.3). In addition, a number of instrument performance tests and calibrations were carried out, including calibration LED exposures, spacecraft rolls designed so that the HI-A and HI-B cameras viewed the same regions of sky for intercalibration, and stray-light off-point tests.

In the stray-light off-point tests, the spacecraft attitude was offset from nominal pointing in a series of steps in HI pitch ranging from $1.5^{\circ}$ towards the Sun to $1^{\circ}$ away from the Sun, enabling the stray-light rejection of the forward baffle to be verified. The results obtained are discussed in Section 9.2.2.

In the course of instrument commissioning, and during the early science mission operations phase, two issues related to the instrument on-orbit performance became apparent. Firstly, as can be seen in Figure 33 of this paper and in Figure 14 of Brown, Bewsher, and Eyles (2009), the PSF of HI-2B is somewhat broader and more asymmetric than that of HI$2 \mathrm{~A}$, resulting in the images of stars being more spread out. In fact this focusing problem was known about before launch, but because of programmatic constraints, together with the fact that it was not expected to impact significantly on the primary science objective of imaging CMEs, it was not deemed necessary to take any remedial action prior to launch. The cause of the problem is most likely a manufacturing or assembly error in the HI-B lens assembly, resulting in incorrect spacing of some of the lens elements - tests using external corrector lenses placed in front of the camera during spacecraft-level testing produced no significant improvement in the imaging performance, indicating that the problem is not due to a simple error in the setting of the focal distance between the lens assembly and the CCD.

As a result of the degraded PSF of HI-2B, the attitude solutions obtained using the background stars in each image (see Brown, Bewsher, and Eyles, 2009) are not as good as they are for the other cameras although they are still completely acceptable - the mean squared deviation between the positions of observed stars and their predicted catalogue positions, averaged over all acceptable images, is 1.48 image pixels for HI-2B compared to 0.78 image pixels for HI-2A. The degraded PSF has a negligible effect on the primary science objectives of the mission since CMEs are diffuse structures that do not show detail at the level of the basic instrumental PSF. However, work is ongoing to model and correct for the PSF, although this is a complex problem because of its asymmetry and variability over the field of view.

The second instrument performance issue that has become apparent is that there are occasional and random discontinuities in the pointing of HI-1B relative to the spacecraft attitude solutions, by up to $\sim 0.1^{\circ}-0.2^{\circ}$. These discontinuities usually manifest themselves as steps in a plot of pointing attitude against time, although in a small proportion of HI-1B images the pointing discontinuities occur during the exposures, resulting in star images that are smeared, trailed, or even split. The origin of these sudden changes in pointing is uncertain - the on-orbit environment should be highly stable, both thermally and mechanically. The most plausible explanation is that since HI-B is facing into the direction of motion of 
Table 9 Summary of prelaunch thermal predictions and on-orbit measured temperatures $\left[{ }^{\circ} \mathrm{C}\right]$.

\begin{tabular}{|c|c|c|c|c|}
\hline \multirow[t]{2}{*}{ Subsystem $^{\mathrm{a}}$} & \multicolumn{2}{|c|}{ Prelaunch predictions } & \multicolumn{2}{|c|}{ On-orbit measurements ${ }^{\mathrm{c}}$} \\
\hline & Cold case & Hot case & HI-A & HI-B \\
\hline Structure & -69 to -53 & -56 to -33 & -70 to -23 & -68 to -29 \\
\hline HI-1 CCD & -84 & -81 & -83 & -80 \\
\hline HI-2 CCD & -83 & -80 & -80 & -76 \\
\hline HI-1 lens barrel ${ }^{b}$ & -28 & -28 & -15 & -29 \\
\hline HI-2 lens barrel ${ }^{b}$ & -28 & -28 & -15 & -15 \\
\hline CEB internal & -18 & -6 & -13 & -13 \\
\hline Wax actuator & -74 & -61 & -72 & -71 \\
\hline
\end{tabular}

${ }^{a}$ Operational requirements apply to all items shown, with the exception of the wax actuator.

${ }^{\mathrm{b}}$ Heaters used to control lens barrel temperatures.

${ }^{\mathrm{c}}$ Measurements taken in January 2007, when spacecraft were almost in their final heliocentric orbits.

STEREO-B, impacts by dust particles on the instrument may be perturbing the pointing of the camera relative to the spacecraft. The degraded PSF of HI-2B has to date prevented any definitive conclusions being reached as to whether the pointing discontinuities also affect this camera (i.e., whether the alignment of the entire instrument is being perturbed or just the HI-1B camera).

The procedure described in Brown, Bewsher, and Eyles (2009) of using the background stars in each image to derive an attitude solution for that image essentially avoids any problems in data analysis owing to the pointing discontinuities - in essence the HI cameras are used as star trackers and the images become self-calibrating. Only in a very small proportion of images (a few percent) where the star images are significantly smeared is it not possible to obtain improved attitude solutions in this way - these images are flagged as "bad" and will not normally be used for science data analysis.

\subsubsection{Instrument Thermal Status after Door Deployment}

Table 9 shows early on-orbit temperature measurements for the two instruments, together with the prelaunch predictions reproduced from Table 6. All the measured temperatures satisfy the requirements stated in Table 4, within acceptable margins. All CCD temperatures are close to the design value of $-80^{\circ} \mathrm{C}$. The setpoint for the lens barrels is higher than that used in prelaunch predictions. The temperatures shown were achieved by using only the operational heater circuits.

For HI-B, although the HI-1 lens barrel temperature is within the requirements in Table 4, the available heater power was not sufficient to raise its temperature to the $-15^{\circ} \mathrm{C}$ setpoint. This was not unexpected since spacecraft-level thermal testing before launch showed a similar anomaly, which is believed to be due to a thermal short or leak that has developed between the lens barrel and housing. The HI-1B lens barrel setpoint was lowered to $-29.5^{\circ} \mathrm{C}$, which is entirely satisfactory and in no way compromises the optical performance.

\subsubsection{Stray-Light Off-Point Calibration Results}

A stray-light off-point calibration was performed on HI-A on 30 January 2007. Exposures were taken at various spacecraft off-point angles up to $1.5^{\circ}$ towards the Sun, and up to $1.0^{\circ}$ 

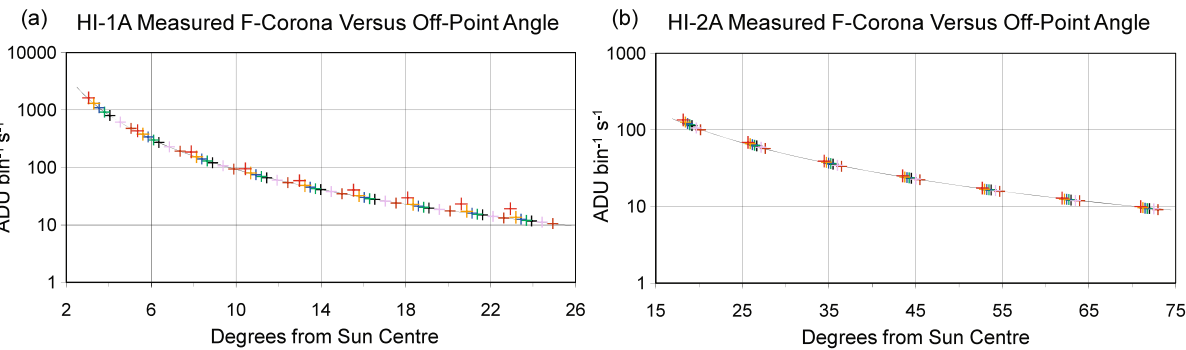

(c) HI-1A (Measured - Profile) Versus Off-Point Angle

(d) HI-2A (Measured - Profile) Versus Off-Point Angle
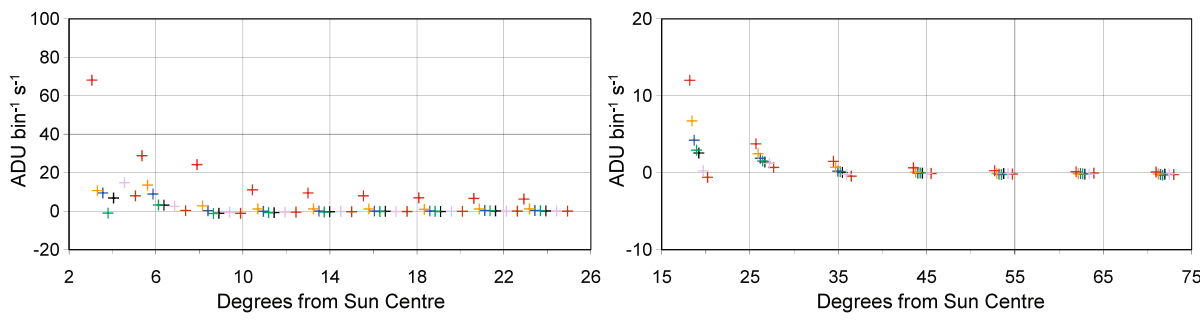

Figure 34 Measured F-coronal intensity plotted against angle from Sun centre for (a) HI-1A and (b) HI-2A. (c) and (d) Residual intensities after subtracting the fitted profiles. The colours of the data points plotted indicate the spacecraft off-point angles as follows: black is nominal pointing; green, blue, orange, and red indicate off-point angles of $0.25^{\circ}, 0.5^{\circ}, 0.75^{\circ}$, and $1.0^{\circ}$ towards the Sun, respectively; purple and brown indicate off-points angles of $0.5^{\circ}$ and $1.0^{\circ}$ away from Sun, respectively. The continuous black line in the upper plots corresponds to the fitted power-law profile.

away from the Sun in HI pitch, relative to nominal pointing. Visual inspection of the images indicated no evidence of any increase in stray light up to $1.0^{\circ}$ offset towards the Sun. At $1.0^{\circ}$ offset, and to a much greater extent at $1.5^{\circ}$ offset, there was a substantial amount of stray light and ghost images evident in both HI-1 and HI-2.

For each off-point, the F-coronal intensity was measured at eight locations across the centerline of the field of view. The values obtained are plotted against distance from the Sun centre (allowing for the off-point angle) in Figures 34(a) and (b). A power-law profile $D^{-n}$ (where $D$ is angular distance from Sun centre and $n$ is a constant index) was fitted to the data points corresponding to nominal pointing, and Figures 34(c) and (d) show the residuals after the fitted profile is subtracted from the data points.

If the stray-light intensity does not change with off-point angle, then all the data points should lie on the same curve. This is clearly the case for all off-points except for $-1.0^{\circ}$ and to a much smaller extent $-0.75^{\circ}$, where the measured values lie significantly above the fitted profiles.

The following conclusions are drawn:

- There is at least $0.5^{\circ}$ margin in the pointing alignment of HI-1A relative to the Sun direction.

- In the nominal case, the stray-light level in HI-1A is less than about $2 \%$ of the F-coronal background across the entire field of view; a similar limit applies to HI-2A.

- The dramatic decrease in stray-light rejection between $1.0^{\circ}$ and $1.5^{\circ}$ offset towards the Sun is as expected based on the geometry of the forward baffles, corresponding to the loss in effectiveness of the first baffle stage. 
Table 10 The exposure and summed image timing parameters for normal synoptic observations.

\begin{tabular}{lll}
\hline & HI-1 & HI-2 \\
\hline Individual exposure time & 40 seconds & 50 seconds \\
Exposure cadence & 60 seconds & 60 seconds \\
Number of images summed & 30 & 99 \\
Total exposure time & 1200 seconds & 4950 seconds \\
Exposure sequence duration & 30 minutes & 99 minutes \\
Summed image cadence & 40 minutes & 2 hours \\
Observing duty cycle & $50 \%$ & $69 \%$ \\
\hline
\end{tabular}

Results for HI-B off-point calibrations were broadly similar except that the margin in the pointing alignment was a little smaller, about $0.3^{\circ}$.

\subsection{HI Science Mission Operations}

The science operations phase for HI commenced at the beginning of April 2007, although observations of scientific importance were made before this time (e.g., Fulle et al., 2007; Harrison et al., 2008). Science operations are relatively simple for HI since the instrument performs a continuous synoptic, or monitoring, observing program. The details of this program were refined during the commissioning phase and the final values arrived at for the exposure times, cadences, etc. are given in Table 10.

The factors determining the choice of values are as follows:

- The overall telemetry allocation available to HI corresponds to an average of two $1024 \times$ 1024 binned images per hour.

- The respective plate scales of the HI-1 and HI-2 cameras and the consequent time evolution of propagating structures result in the choice of 40-minute and 2-hour cadences for the summed images.

- The exposure sequence duration is chosen so that the drift of the star field through the field of view $\left(\approx 2.5\right.$ arc-min hour $^{-1}$ ) corresponds to $\approx 1$ image bin, thereby ensuring that the star images are not significantly smeared over the sequence.

- The individual image exposure times are chosen from dynamic range considerations. In the case of HI-1 the brightest region of the F-corona corresponds to about $60 \%$ of the dynamic range there are usually no saturated star images, although Venus and Mercury, if present, are significantly saturated. For HI-2 there are typically $\sim 2-10$ saturated star images in the field of view (in addition to any planets that are present).

In addition to the 36 summed images taken for HI- 1 and 12 for HI- 2 every 24 hours, one full-resolution $(2048 \times 2048$ pixels $)$ single exposure is normally taken with each camera for performance monitoring.

Momentum dumps are performed at approximately 6- to 8-week intervals on each spacecraft to off-load the reaction wheels in the attitude control system. This operation interrupts normal observations, although camera calibration images are taken at these times. Synoptic observations are also interrupted occasionally for spacecraft high-gain antenna calibrations and calibration rolls for the SCIP instruments. However, with the exception of these occasional interruptions the synoptic observing program detailed here is performed continuously throughout the mission. 


\subsection{STEREO Space Weather Beacon Data}

The synoptic observing programme images from the SECCHI instruments are available on the various Web sites with a latency of 2-3 days. To facilitate space weather forecasting, the STEREO spacecraft have a Space Weather Beacon mode, which is a continuous, realtime, low-data-rate (633 bits per second) transmission of highly compressed data from the STEREO instruments.

In the case of HI, one summed image from each camera is sent every 2 hours via the beacon mode. The images are $256 \times 256$ binned and highly compressed.

\section{Data Processing and Analysis}

Data from the SECCHI instruments aboard STEREO, including the Heliospheric Imagers, are available for downloading from several Web sites, including the UK Solar System Data Centre (UKSSDC), based at RAL (http://stereo.rl.ac.uk). The HI data are available in two forms: Level 0.5 and Level 1.

Level 0.5 (L0.5) data are raw, uncalibrated instrument images that have been extracted from the down-linked spacecraft telemetry and made available in the Flexible Image Transport System (FITS; Wells, Greisen, and Harten, 1981) file format. The image is a 2D array of the appropriate size, and there is a header that contains a large number of keywords to specify all the instrument and spacecraft parameters relevant to the observation. Additionally, in the case of summed HI science images there is an extended header, which is a table giving parameters of the individual exposures in the image sequence (e.g., the exposure times and durations for the individual exposures). The full definition of the L0.5 keywords is given in $\mathrm{ftp} / / /$ louis14.nrl.navy.mil/pub/secchi/ssw/doc/FITS_keywords.pdf.

Level 1 (L1) data have been processed using SECCHI_PREP, which is an IDL routine available in the SolarSoftWare distribution (Freeland and Handy, 1998). SECCHI_PREP reads in L0.5 images and then applies the latest image correction and calibration procedures for all the SECCHI instruments. The procedures applied to HI images are described in the subsequent sections.

The effects of the image processing performed by SECCHI_PREP (Section 10.1), followed by the background subtraction (Section 10.2), are illustrated in Figure 35.

\subsection{Processing of HI images by SECCHI_PREP}

There are options for enabling and disabling various functions, but in the default case SECCHI_PREP performs the following operations on L0.5 data to produce L1 files:

- Remove the cosmic-ray scrubbing values (see Section 5.3) from the final row of the image, replacing the values in these bins with values from the adjacent row.

- Identify saturated columns and missing data blocks, and insert appropriate data values.

- Apply the correction for shutterless readout of the cameras.

- Apply a flat-field correction to the image.

- Correct the pointing and optics parameter values in the headers.

\subsubsection{Saturated Columns and Missing Data Blocks}

When the signal from a bright object, such as a planet, exceeds the full well capacity of a pixel, the excess charge bleeds into adjacent pixels in the same column, causing vertical columns of saturated pixels. Bleeding of excess charge horizontally across columns is 

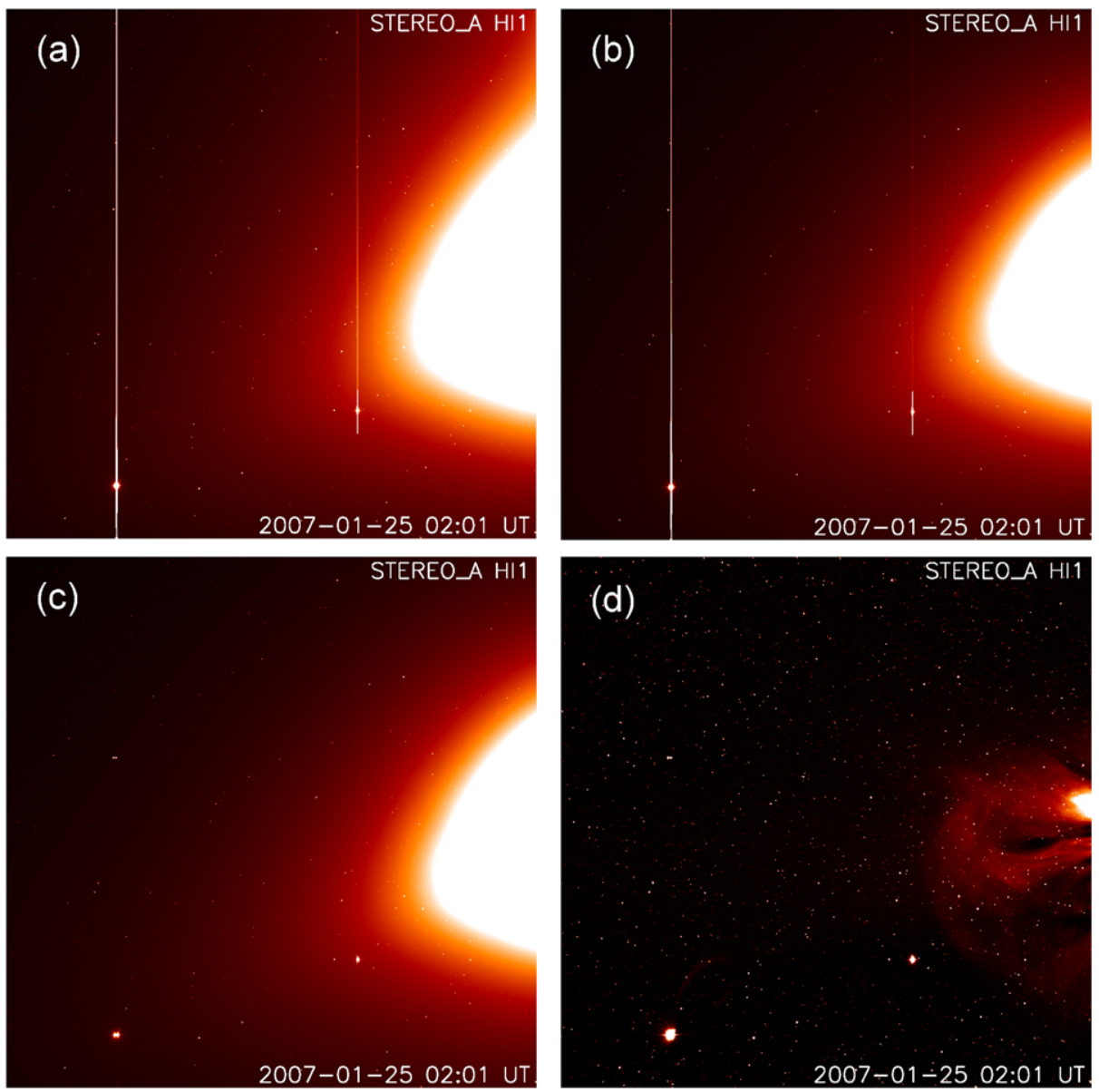

Figure 35 An image from HI-1A taken on 25 January 2007, illustrating the effects of various stages of image processing: (a) the raw image, (b) SECCHI_PREP has been used to apply corrections for shutterless operation and the flat field, (c) the residual "blooming" caused by saturation around the planets Venus and Mercury has been removed, and (d) the background has been subtracted to reveal a CME.

inhibited by the channel stops in the CCD structure, although in extreme cases where the saturated column reaches the output register the charge may then bleed across the register and up into the adjacent columns.

Since the presence of saturation anywhere in a column invalidates the shutterless correction (see next section), all data in the column must be regarded as potentially invalid. SECCHI_PREP identifies this situation and marks all pixels accordingly by replacing the pixel ADU values with NaN. SECCHI_PREP also identifies missing blocks in an image (from telemetry dropouts) and inserts appropriate values in these pixels.

\subsubsection{Correction for Shutterless Readout}

Mainly because of mechanical accommodation constraints, the HI cameras do not have shutters and since the CCDs are full-frame format they remain exposed to the sky scene during the image readout process, and also during the clear process prior to each exposure. Since 
Figure 36 Drawing illustrating image smearing during the readout process, resulting from the shutterless operation of the HI cameras.

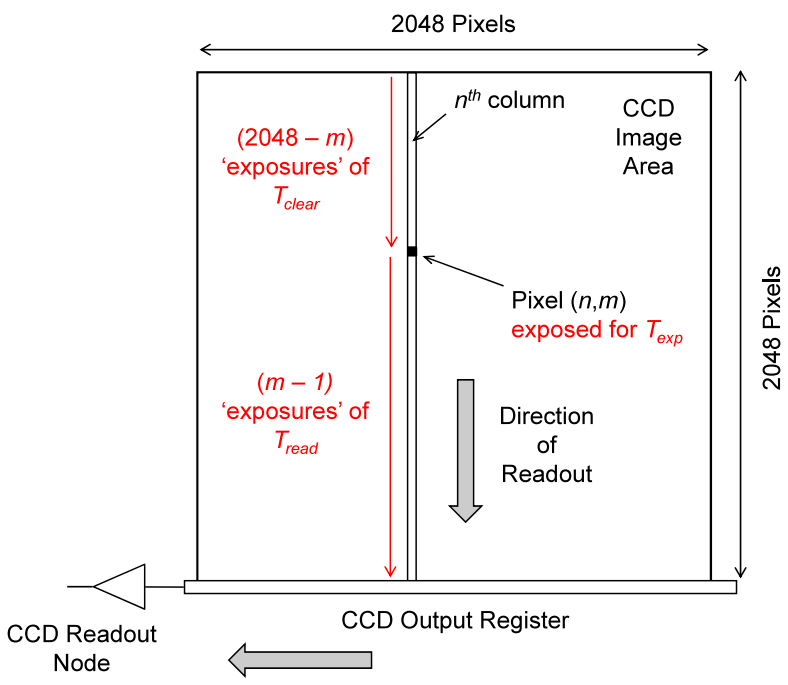

the readout time of an image in particular is not insignificant compared with the typical exposure times, this results in some smearing of the images during the readout.

Consider CCD pixel $(n, m)$ as shown in Figure 36. After an exposure time $T_{\text {exp }}$, the image is read out by transferring each line in turn into a horizontal output register and then clocking each pixel serially out of the horizontal register. If the line transfer time during the readout is $T_{\text {read }}$, then there will be a "pseudo exposure" of duration $T_{\text {read }}$ at every line position down the column, under the pixel location. Hence, in addition to the nominal exposure of the pixel there will be $(m-1)$ exposures of duration $T_{\text {read }}$. This means that there will be a gradient of effective exposure time increasing vertically up the image and also, since these additional exposures are to different regions in the sky scene, there will be a vertical smearing of the resultant image. Bright objects such as stars or planets will have vertical "trails" above their locations in the image.

In addition, prior to each exposure the CCD is cleared by clocking residual charge downwards into a "dump drain" in a similar manner to the image readout, albeit at a faster rate. Consequently, for pixel $(n, m)$ we must also add contributions from the $(2048-m)$ locations above the nominal location, each with an exposure time $T_{\text {clear }}$ corresponding to the line transfer time during the clear process. This causes vertical smearing and trailing of stars in the opposite direction.

In practice, $T_{\text {read }}$ dominates over $T_{\text {clear }}$, their values being 2350 and $124 \mu$ s, respectively, in the nominal camera readout mode. The total time to read out an image, including overheads, is approximately 4.837 seconds.

Expressing this mathematically, the total response $R$ for pixel $(n, m)$ is given by

$$
R(n, m)=\left[T_{\exp } \times I(n, m)\right]+\sum_{y=m+1}^{2047}\left[T_{\text {clear }} \times I(n, y)\right]+\sum_{y=0}^{m-1}\left[T_{\text {read }} \times I(n, y)\right],
$$

where the count rate for pixel $(x, y)$ is given by $I(x, y)$. This can be expressed rather more succinctly by the matrix relationship

$$
\mathbf{R}=\mathbf{T I},
$$


where $\mathbf{T}$ is a $(2048 \times 2048)$ matrix filled with the value $T_{\exp }$ along the diagonal, the value $T_{\text {read }}$ in all elements above the diagonal, and $T_{\text {clear }}$ in all elements below the diagonal. Hence, the image that is read out may be corrected for the effects of the shutterless operation of the camera by the matrix multiplication

$$
\mathbf{I}=\mathbf{T}^{-1} \mathbf{R} .
$$

It should be noted that the corrected image $\mathbf{I}$ has units of count rate (i.e., $\mathrm{ADU} \mathrm{s}^{-1}$ ), rather than total signal in ADU. Indeed, since the effective exposure time varies by nearly 5 seconds down the CCD it is not meaningful to convert the image to total signal.

Although this procedure normally completely removes the effects of the shutterless readout, there are two limitations to its validity. Firstly, if there is a cosmic-ray hit on the CCD during the exposure this will produce a bright region without the trailing along the associated column(s) during the readout. Applying the shutterless correction will then produce a dark trail in the columns. This is not an issue for the summed science images since cosmic-ray hits are removed efficiently by the onboard scrubbing, but the effect is noticeable in singleexposure images. Secondly, if saturation occurs anywhere in a column then the signal in the affected pixels does not represent the true incident light intensity, so again the correction is not valid.

\subsubsection{Flat-Field Corrections and Photometric Conversions}

To apply a flat-field correction to the L1 images, SECCHI_PREP multiplies the L0.5 image by a calibration image (after performing the shutterless correction). Deviations from uniform response may occur for the following reasons:

- There can be variation in the efficiency of the optics across the field of view owing to changes in transmission of the optical elements and coatings, geometrical aperture effects, and vignetting. These effects, which generally produce large-scale variations, were characterised to a certain extent during prelaunch calibrations (see Section 8.4.2).

- CCDs generally show pixel-to-pixel variations in response, typically of the order of a few percent. These contribute a small-scale variation; in general there is no significant large-scale nonuniformity in CCD response.

- Debris, such as dust particles, on the CCD will cause nonuniformity of response, generally on fairly small spatial scales (although condensation of volatile materials may cause largescale effects).

As of October 2008, SECCHI_PREP applies the large-scale geometrical corrections described in Section 8.4.2. These are reasonable in the circular part of the field of view but are significantly in error in the corners, because the optics suffer significant vignetting in these regions, whilst the corrections have simply been extrapolated (since there were no applicable data from the prelaunch calibrations). In addition, there is a correction to the response in rows 512, 1024, and 1536 of the CCD, corresponding to the so-called stitch lines. During the manufacturing process, the CCD pixel structure is photo-composed by replicating four times over a basic pattern of $512 \times 2048$ pixels, resulting in nonuniformity of response at the joints. There is no photometric conversion implemented at present.

At the time of writing, work is ongoing to use the response to stars drifting through the fields of view to produce improved large-scale flat-field corrections, together with photometric response calibrations (Bewsher et al., in preparation). Small-scale flat-field corrections will follow later. 


\subsubsection{Updating of Pointing and Optics Parameters}

Until recently (August 2008), the pointing attitude parameters in the FITS headers were derived from the spacecraft attitude solutions, together with refined prelaunch values for the offsets between the HI telescopes and the spacecraft reference axes. This has not been entirely satisfactory for the following reasons:

- Although the Sun-pointing $(+X)$ spacecraft axis is maintained pointing accurately towards Sun centre by the SECCHI GT, the roll angle about this axis is derived from the spacecraft star trackers and inertial measurement unit and is subject to significant errors and uncertainty.

- Images from HI-1B show occasional and irregular discontinuities in pointing, by up to $0.1^{\circ}-0.2^{\circ}$ (see Section 9.2).

The background stars in the HI images are now used to obtain optimised attitude solutions for all cameras on an image-by-image basis (Brown, Bewsher, and Eyles, 2009). Typically, between 20 and 80 stars are used in the optimisations and good solutions are obtained for the overwhelming majority of images (with an average error in residuals of fitted star positions of $<1$ image pixel, except for HI-2B where it is $<2$ image pixels). This removes the uncertainties in the solutions derived from spacecraft pointing and the effects of the pointing discontinuities in HI-B.

Since August 2008, SECCHI_PREP updates the pointing keywords in the L1 image headers using the attitude solutions obtained from the background stars (where available). Updated values for the optical parameters $f_{\mathrm{p}}$ and $\mu$ (see Section 8.4.1), which were obtained as a result of the star fitting, are also inserted in the L1 headers.

\subsection{Background Subtraction}

After the images have been corrected and calibrated using SECCHI_PREP, the F-coronal background must be removed to reveal transient features such as CMEs. This is possible because the F-coronal background is essentially constant on time scales characteristic of the evolution of CMEs. Two alternative approaches are used to remove the background: the "running difference" method and the subtraction of a mean coronal background measured over a sequence of images.

The running difference method simply involves taking the difference between each image and the preceding one, on a pixel-by-pixel basis. This provides a simple "quick look" way of searching for coronal transients - propagating CMEs are revealed as bright regions followed by adjacent dark regions - although it is not compatible with quantitative photometry.

The coronal background signal can be calculated by taking the minimum intensity measured in each pixel over a sequence of images. Stars drift across the field of view (typically by $\approx 1$ image pixel between consecutive synoptic images) and so do not appear in the background image. Larger scale features, such as the Milky Way, require a longer sequence of images. Appropriate time scales for the calculation of a mean background vary from 1 day to several days, up to a maximum of 27 days.

To calculate and then subtract a valid background it is necessary that the measured coronal background signal remains constant over the time scale involved. This implies that the instrument pointing attitude must be stable relative to the large-scale coronal structure. Two specific problems arise for the HI data:

- The spacecraft roll angle may change relative to the ecliptic. This applies particularly to STEREO-B early in the mission. The effect is that the F-corona rotates across the field of view, thereby invalidating attempts to calculate and subtract a constant background. 
- The pointing discontinuities of HI-1B have a similar effect. The gradient of the F-corona along the ecliptic is such that an error of only $1 / 3$ of a pixel in pointing will leave a residual background signal comparable with the intensity of the transient solar wind features.

These effects can be allowed for by correcting the pointing attitudes of the images in the sequence before determining the minimum for each pixel (i.e., the images are first shifted and rotated into a fixed orientation relative to the large-scale corona). Procedures to do this by using the optimised attitude solutions obtained from the background star fields are currently being developed, and background estimates over a range of time scales from 1 day up to 27 days are being derived.

\section{Results}

The HI instruments have been operational since early 2007 and have already detected numerous CMEs, as evidenced by the HI event list, which is maintained on the RAL STEREO Web site (http://stereo.rl.ac.uk).

In addition, many outstanding discoveries and serendipitous observations relevant to the additional scientific objectives of the mission have been made, including the following:

- the discovery of the atomic ion tail of Comet McNaught (Fulle et al., 2007),

- a direct observation of the interaction between a CME and a comet (Comet Encke), leading to the complete disconnection of the comet's plasma tail (Vourlidas et al., 2007),

- observations of the structure of the solar wind, in particular the first direct white-light images of corotating interaction regions in the interplanetary medium (Rouillard et al., 2008; Sheeley et al., 2008a, 2008b),

- the first observation of the planetary impact of a CME by the STEREO spacecraft confirmed by in situ measurements by the magnetometer aboard the Venus Express spacecraft (Rouillard et al., 2009), and

- stellar variability studies (Bewsher et al., in preparation).

One of the first CMEs to be observed by HI was an event seen in HI-1A between 14:00 UT on 24 January and 04:00 UT on 25 January 2007. The event was first observed by LASCO C2 and $\mathrm{C} 3$ and was followed through $\mathrm{HI}-1 \mathrm{~A}$ out to about $50 R_{\odot}$ (in the plane of the sky) over a total period of 15 hours. Unfortunately, there was a data gap on HI-1A after 04:00 UT, but the event was detected subsequently by HI-2A and later by SMEI on 2628 January. At the time of the observations both STEREO spacecraft were still very close to the Earth, with viewing locations only just outside the Sun - Earth line. Consequently, the event was not observed by the HI-B instruments.

Detailed analysis of this event, including comparison with other instruments and brief descriptions of other early CME events observed by HI, was reported by Harrison et al. (2008). Here we present the essential observations to demonstrate that $\mathrm{HI}$ is easily meeting its requirements specifications in terms of CME detection.

Figure 37 shows an image of the event from HI-1A early on 25 January, the image being the sum of 25 exposures, each of 24-second duration. The raw image was first corrected for the shutterless operation of the camera and then the F-coronal background was removed, as described in Section 10. The background was determined by taking the minimum intensity for each image pixel over a number of similar images around the same time.

The quality of the background-subtracted image is outstanding and demonstrates the effectiveness of the image processing, with stars down to 12th to 13th magnitudes visible. There is no evidence of the brighter stars "trailing", demonstrating the efficiency of the 


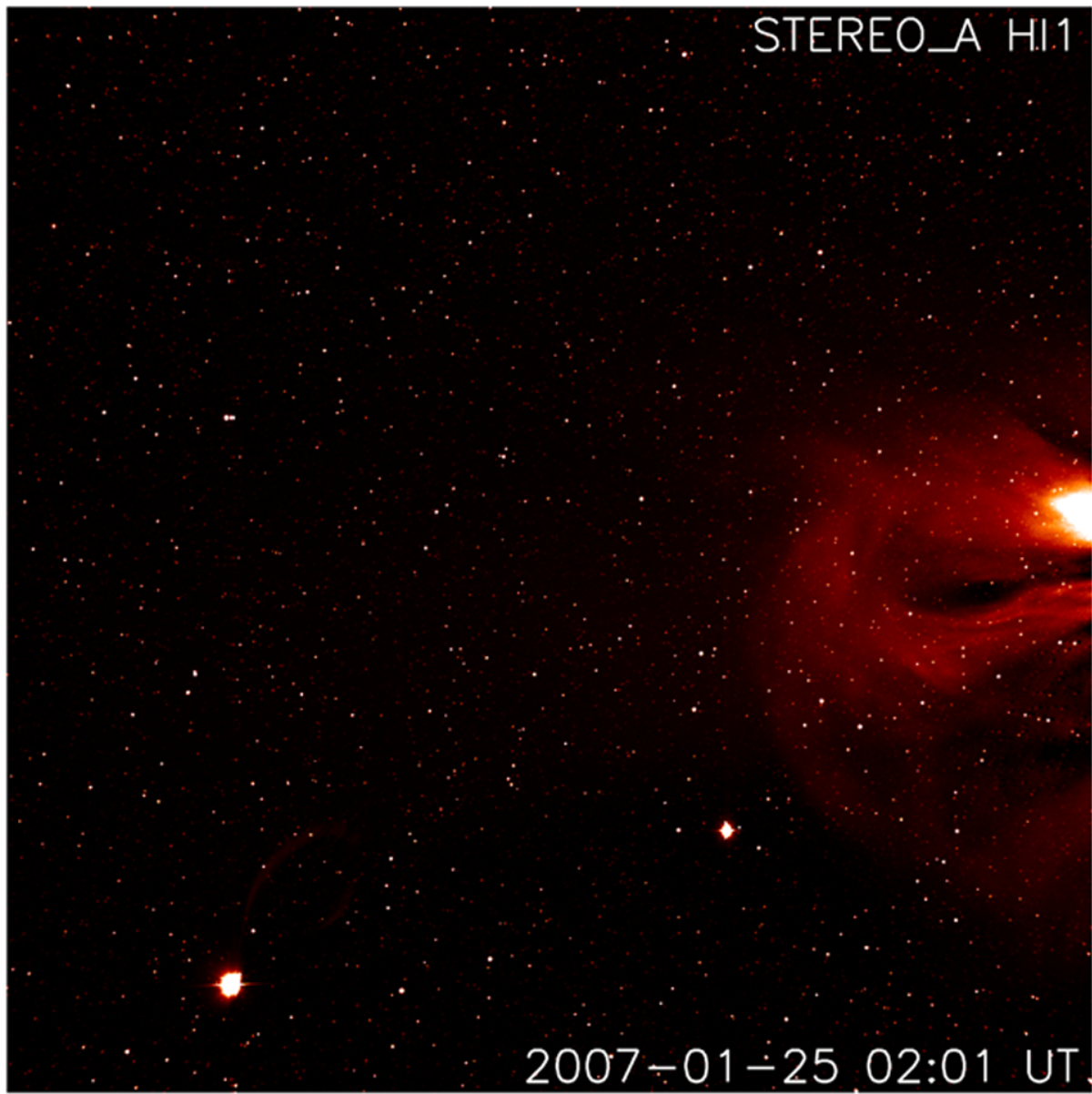

Figure 37 A HI-1A image taken on 25 January 2007. The image is $20^{\circ}$ across and the Sun is $4^{\circ}$ off the right-hand edge. The F-coronal background has been subtracted to reveal a CME expanding across the right-hand side of the image. Stars down to 12th to 13th magnitudes are visible, together with the bright images of Venus (near bottom left) and Mercury.

shutterless correction. Pixel values in the saturated columns associated with Venus and Mercury have been replaced with average values from adjacent columns (since the saturated columns do not contain any valid data). The structure of the CME expanding away from the east limb of the Sun at around $600 \mathrm{~km} \mathrm{~s}^{-1}$ is clearly visible. The presence of Venus and Mercury in the field of view graphically demonstrates the potential for combining remote sensing observations with in situ measurements.

Figure 38(a) shows the profile of the raw intensity measured across the central row of the HI image, before subtraction of the F-coronal background. The profile is dominated by the F-coronal signal with small spikes resulting from stellar images.

Figure 38(b) shows a plot of the residual signal after the coronal background has been subtracted. A number of stars are visible but the peak of the CME front is clearly visible above a slowly varying residual component of the F-corona, which has not been completely subtracted out. In Figure 38(c) the median of the central 25 rows of the background- 

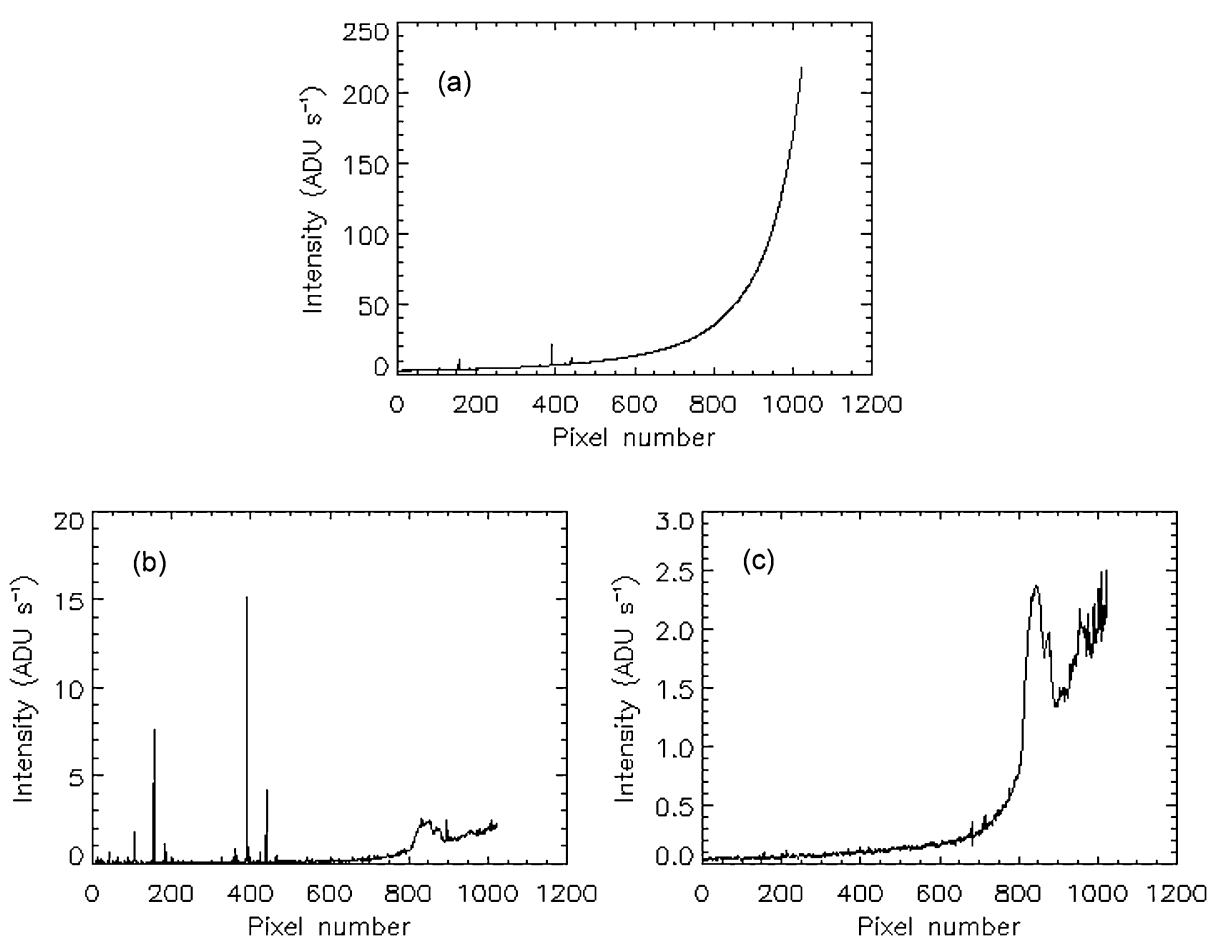

Figure 38 (a) The intensity profile plotted across the central row of the HI image, before subtraction of the F-coronal background, (b) the same plot after subtraction of the background, and (c) the profile of the median of the 25 central rows across the background-subtracted image.

subtracted image is plotted. Here the spikes from stellar images are removed and the structure of the CME stands out very prominently above the residual F-coronal signal. The structure near the right-hand edge of the profile (close to the Sun) is probably associated with streamer belts in the K-corona superimposed on the residual F-corona.

Both Figures 38(b) and (c) show that the peak intensity of the CME is $\approx 2.3 \mathrm{ADU} \mathrm{s}^{-1}$ and that structure associated with the $\mathrm{CME}$ is clearly distinguishable down to at least $\approx 0.2 \mathrm{ADU} \mathrm{s}^{-1}$. Referring back to Figure 38(a) we can see that the corresponding F-coronal signal in this part of the image is about $50 \mathrm{ADU} \mathrm{s}^{-1}$ and so the peak intensity of the CME front is $4.5 \%$ of the coronal background, and features $\approx 0.5 \%$ of the background are visible. Crucially, we have demonstrated that we can readily detect CME features down to less than $1 \%$ of the F-coronal intensity. These measurements were made at about $40 R_{\odot}$ (in the plane of the sky). In terms of solar brightness, it is estimated that the peak intensity in the CME is $\approx 7.5 \times 10^{-14} B_{\odot}$ and the data demonstrate that we can detect features down to less than $10^{-14} B_{\odot}$ at these distances from the Sun (Harrison et al., 2008).

\section{Summary and Conclusions}

We have described the design, development, and operation of the Heliospheric Imagers, unique instruments that are now operating successfully in orbit aboard the two NASA STEREO spacecraft. Meticulous attention to detail in the design, development, and manu- 
facturing have enabled the demanding requirements specifications to be achieved, including rejection of solar background light to levels of $\sim 10^{-13}$ to $10^{-14} B_{\odot}$.

A careful programme of ground tests and calibrations at both the subsystem and the instrument level, together with in-orbit verifications, has demonstrated that the requirements have been met. The early CME results demonstrated the ability to extract the CME signal at less than $1 \%$ of the F-coronal background.

In addition to observations of CMEs and solar transient events, the HI instruments have already made a number of other highly significant scientific observations.

At the time of writing (September 2008) the total separation angle between the STEREO spacecraft at the Sun is approximately $70^{\circ}$ and the mission is entering the optimum phase for observations of CMEs propagating along the Sun-Earth line using the HI instruments at the two widely separated viewing points.

Acknowledgements The Heliospheric Imager instrument was developed by a collaboration that included the University of Birmingham and the Rutherford Appleton Laboratory, both in the UK, the Centre Spatial de Liège (CSL), Belgium, and the U.S. Naval Research Laboratory (NRL), Washington DC, USA. The STEREO/SECCHI project is an international consortium of the Naval Research Laboratory (USA), Lockheed Martin Solar and Astrophysics Lab (USA), NASA Goddard Space Flight Center (USA), Rutherford Appleton Laboratory (UK), University of Birmingham (UK), Max-Planck-Institut für Sonnensystemforschung (Germany), Centre Spatial de Liège (Belgium), Institut d'Optique Théorique et Appliquée (France), and Institut d'Astrophysique Spatiale (France). The USA institutions were funded by NASA; the UK institutions by Physics and Astronomy Research Council (PPARC; now the Science and Technology Facilities Council); the German institutions by Deutsche Zentrum für Luft- und Raumfahrt e.V. (DLR); the Belgian institutions by Belgian Federal Science Policy Office; and the French institutions by Centre National d'Etudes Spatiales (CNES) and the Centre National de la Recherche Scientifique (CNRS). The NRL effort was also supported by the USAF Space Test Program and the Office of Naval Research. The authors also wish to acknowledge the strong support of the STEREO Program Office and others at NASA/GSFC throughout the project and particularly during the later stages of instrument development and during spacecraft AIT.

\section{References}

Brown, D.S., Bewsher, D., Eyles, C.J.: 2009, Solar Phys. 254, 185.

Calabretta, M.R., Greisen, E.W.: 2002, Astron. Astrophys. 395, 1077.

Defise, J.-M., Halain, J.-P., Mazy, E., Rochus, P., Howard, R.A., Moses, J.D., Socker, D.G., Simnett, G.M., Webb, D.F.: 2001, Proc. SPIE 4498, 63.

Defise, J.-M., Halain, J.-P., Mazy, E., Rochus, P., Howard, R.A., Moses, J.D., Socker, D.G., Harrison, R.A., Simnett, G.M.: 2003, Proc. SPIE 4853, 12.

Eyles, C.J., Simnett, G.M., Cooke, M.P., Jackson, B.V., Buffington, A., Hick, P.P., Waltham, N.R., King, J.M., Anderson, P.A., Holladay, P.E.: 2003, Solar Phys. 217, 319.

Eyles, C.J., Davis, C.J., Harrison, R.A., Waltham, N.R., Halain, J.-P., Mazy, E., Defise, J.-M., Howard, R.A., Moses, D.J., Newmark, J., Plunkett, S.: 2007, Proc. SPIE 6689, 668907.

Freeland, S.L., Handy, B.N.: 1998, Solar Phys. 182, 497.

French, M.J., Waltham, N.R., Newton, G.M., Wade, R.: 1998, Proc. SPIE 3355, 547.

Fulle, M., Leblanc, F., Harrison, R.A., Davis, C.J., Eyles, C.J., Halain, J.-P., Howard, R.A., Bockelee-Morvan, D., Cremonese, G., Scarmato, T.: 2007, Astrophys. J. 661, L93.

Gopalswamy, N., Mewaldt, R., Torsti, J. (eds.): 2006, Solar Eruptions and Energetic Particles, Geophys. Monogr. Ser. 165, AGU, Washington.

Harrison, R.A.: 2006, In: Gopalswamy, N., Mewaldt, R., Torsti, J. (eds.) Solar Eruptions and Energetic Particles, Geophys. Monogr. Ser. 165, AGU, Washington, 73.

Harrison, R.A., Davis, C.J., Eyles, C.J.: 2005, Adv. Space Res. 36, 1512.

Harrison, R.A., Davis, C.J., Eyles, C.J., Bewsher, D., Crothers, S., Davies, J.A., Howard, R.A., Moses, D.J., Socker, D.G., Halain, et al.: 2008, Solar Phys. 247, 171.

Howard, R.A., Moses, J.D., Socker, D.G.: 2000, Proc. SPIE 4139, 259.

Howard, R.A., Michels, D.J., Sheeley, N.R., Koomen, M.J.: 1982, Astrophys. J. 263, L101.

Howard, R.A., Moses, J.D., Vourlidas, A., Newmark, J.S., Socker, D.G., Plunkett, S.P., Korendyke, C.M., Cook, J.W., Hurley, A., Davila, J.M., et al.: 2008, Space Sci. Rev. 136, 67. 
Jackson, B.V., Buffington, A., Hick, P.P., Altrock, R.C., Figueroa, S., Holladay, P.E., Johnson, J.C., Kahler, S.W., Mozer, J.B., Price, S., et al.: 2004, Solar Phys. 225, 177.

Kim, R.-S., Cho, K.-S., Moon, Y.-J., Kim, Y.-H., Yi, Y., Dryer, M., Bong, S.-C., Park, Y.-D.: 2005, J. Geophys. Res. 110, A11104.

Koutchmy, S., Lamy, P.: 1985, In: Giese, R.H., Lamy, P. (eds.) Properties and Interactions of the Interplanetary Dust, IAU Colloq. 85, Reidel, Dordrecht, 63. ISBN 90-277-2115-7.

Lara, A., Gopalswamy, N., Xie, H., Mendoza-Torres, E., Perez-Eriquez, R., Michalek, G.: 2006, J. Geophys. Res. 111, A06107.

Rouillard, A.P., Davies, J.A., Forsyth, R.J., Rees, A., Davis, C.J., Harrison, R.A., Lockwood, M., Bewsher, D., Crothers, S., Eyles, C.J., et al.: 2008, Geophys. Res. Lett. 35, L10110.

Rouillard, A.P., Savani, N., Davies, J.A., Lavraud, B., Forsyth, R.J., Morley, S.K., Opitz, A., Sheeley, N.R., Sauvaud, J.-A., Simunac, K.D.C. et al.: 2009, Solar Phys. submitted.

Sheeley, N.R., Herbst, A.D., Palatchi, C.A., Wang, Y.-M., Howard, R.A., Moses, J.D., Vourlidas, A., Newmark, J.S., Socker, D.G., Plunkett, S.P., et al.: 2008a, Astrophys. J. 674, L109.

Sheeley, N.R., Herbst, A.D., Palatchi, C.A., Wang, Y.-M., Howard, R.A., Moses, J.D., Vourlidas, A., Newmark, J.S., Socker, D.G., Plunkett, S.P., et al.: 2008b, Astrophys. J. 675, 853.

Socker, D.G., Howard, R.A., Korendyke, C.M., Simnett, G.M., Webb, D.F.: 2000, Proc. SPIE 4139, 284.

Vourlidas, A., Davis, C.J., Eyles, C.J., Crothers, S.R., Harrison, R.A., Howard, R.A., Moses, D.J., Socker, D.G.: 2007, Astrophys. J. 668, L79.

Waltham, N.R., Eyles, C.J.: 2007, Proc. SPIE 6689, 668908.

Wells, D.C., Greisen, E.W., Harten, R.H.: 1981, Astron. Astrophys. Suppl. 44, 263. 